2014

\title{
A THEORETICAL STUDY OF INTERACTION OF NANOPARTICLES WITH BIOMOLECULE
}

Chunhui Liu

Michigan Technological University

Follow this and additional works at: https://digitalcommons.mtu.edu/etds

Part of the Biophysics Commons, and the Physics Commons Copyright 2014 Chunhui Liu

\section{Recommended Citation}

Liu, Chunhui, "A THEORETICAL STUDY OF INTERACTION OF NANOPARTICLES WITH BIOMOLECULE", Dissertation, Michigan Technological University, 2014.

https://doi.org/10.37099/mtu.dc.etds/816

Follow this and additional works at: https://digitalcommons.mtu.edu/etds

Part of the Biophysics Commons, and the Physics Commons 


\title{
A THEORETICAL STUDY OF INTERACTION OF NANOPARTICLES WITH BIOMOLECULE
}

\section{By}

Chunhui Liu

\begin{abstract}
A DISSERTATION
Submitted in partial fulfillment of the requirements for the degree of DOCTOR OF PHILOSOPHY

In Physics
\end{abstract}

MICHIGAN TECHNOLOGICAL UNIVERSITY

2014 

This dissertation has been approved in partial fulfillment of the requirements for the Degree of DOCTOR OF PHILOSOPHY in Physics.

Department of Physics

\author{
Dissertation Advisor: $\quad$ Ravindra Pandey \\ Committee Member: $\quad$ Donald Beck \\ Committee Member: $\quad$ Maximilian Seel \\ Committee Member: $\quad$ Loredana Valenzano
}

Department Chair: Ravindra Pandey. 


\section{Dedication}

To my Mother, my Wife, teachers and Friends 


\section{Contents}

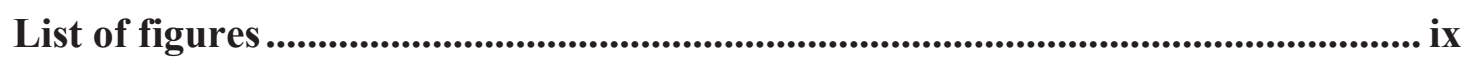

List of tables.............................................................................................................. xiii

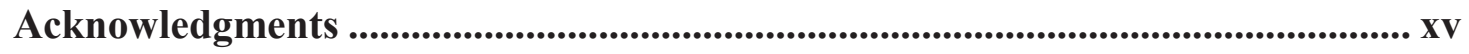

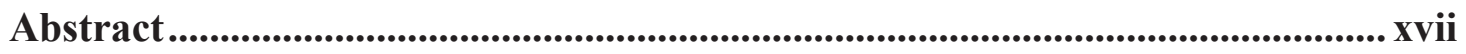

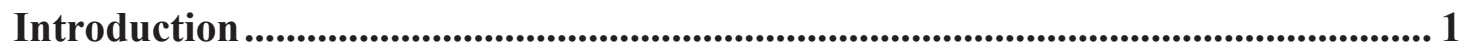

Electronic Structure............................................................................................................... 11

2.1 SCHRÖDINGER EQUATION AND BORN-OPPENHEIMER APPROXIMATION ............... 11

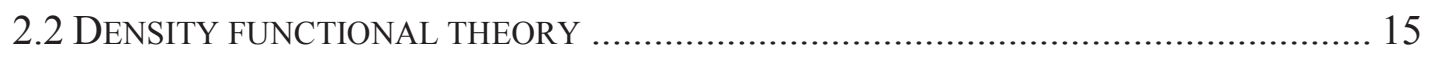

2.2.1 Thomas-Fermi theory and related models............................................. 16

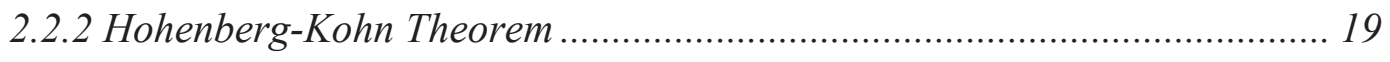

2.2.3 Exchange and correlation functional forms .............................................. 23

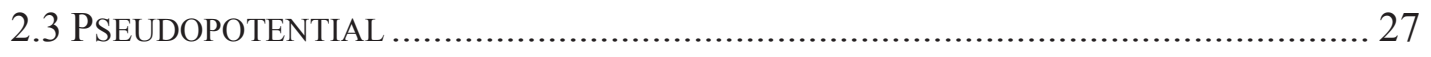

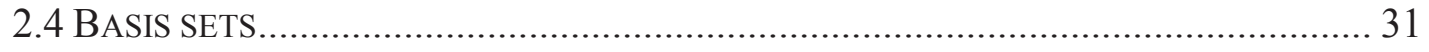

2.5 VienNa AB INiTio Simulation PaCKage (VASP) ............................................ 36

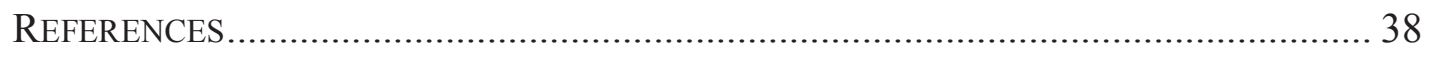

Interaction of Semiconducting Quantum Dots with Dopamine and DNA

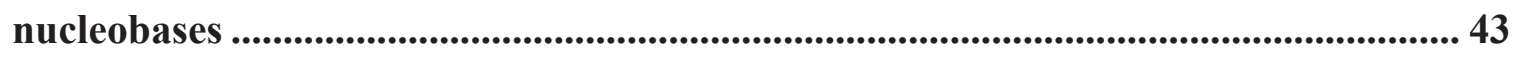




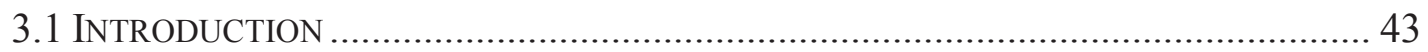

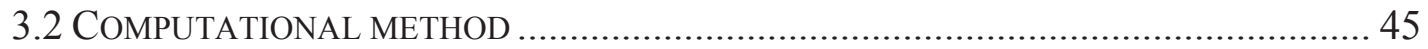

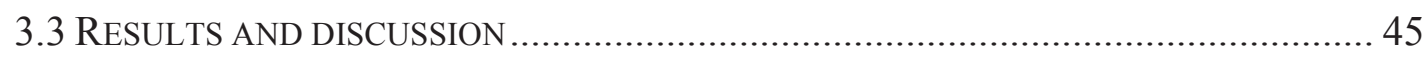

3.3.1 DNA nucleobase molecules .............................................................. 45

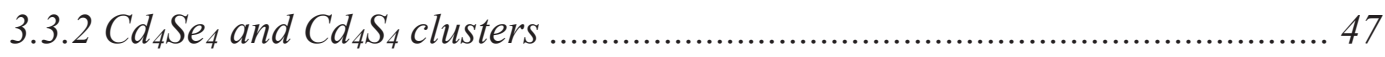

Figure 3.2: A ball and stick model for the $\mathrm{Cd}_{4} \mathrm{Se}_{4}$ and $\mathrm{Cd}_{4} \mathrm{~S}_{4}$ clusters................ 47

3.3.3 Interaction of Cd4Se4 and Cd4S4 QDs with dopamine ........................... 48

3.3.4 Interaction of $\mathrm{Cd}_{4} \mathrm{Se}_{4}$ and $\mathrm{Cd}_{4} \mathrm{~S}_{4} \mathrm{QDs}$ with DNA Nucleobases................... 52

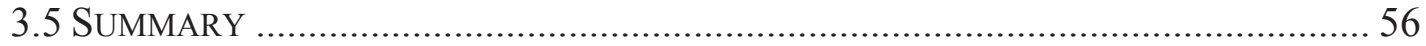

Structure of Membrane Lipids: DMPC and DMPE........................................ 63

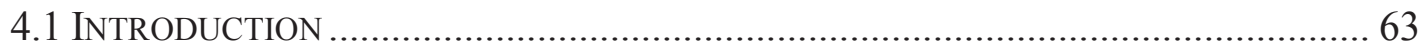

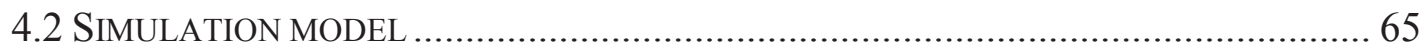

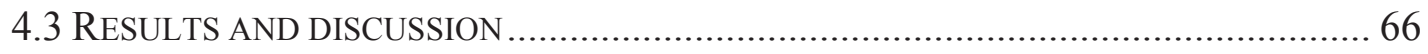

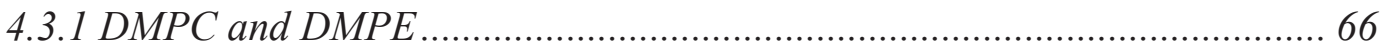

4.3.2 Polar headgroups of DMPC and DMPE....................................... 71

4.3.3 Hydration effects: DMPC and DMPE ............................................... 73

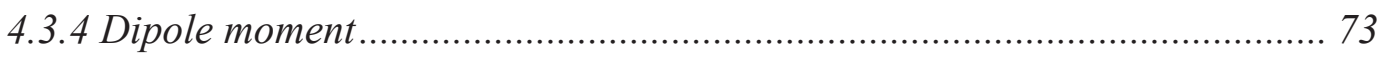

4.3.5 Electron density and Bader charge ................................................. 75

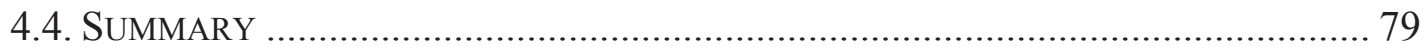

Prediction of Peptide Crystal Structures ............................................................ 87

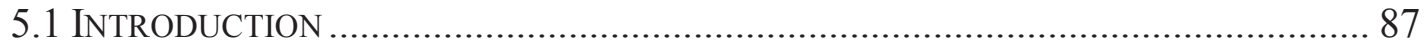




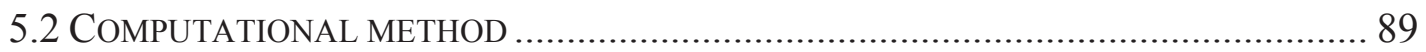

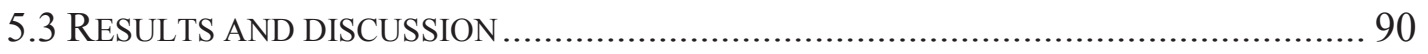

5.3.1 The cyclo (S-Met-S-Met) molecule ..................................................... 90

5.3.2 The cyclo (S-Met-S-Met) crystal ...................................................... 90

5.3.2.2 Step-II: Choice of orientation of two groups in the crystalline structure. 94

5.3.3 Comparison with experiments ........................................................ 101

5.3.4 The enantiomer - cyclo (R-Met-R-Met) crystal.................................. 107

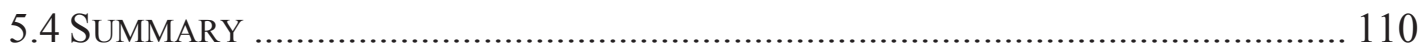

Molecular Dynamics Method ............................................................................. 117

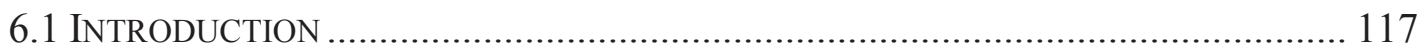

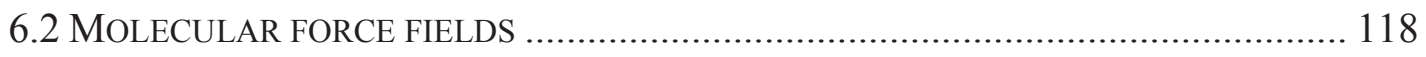

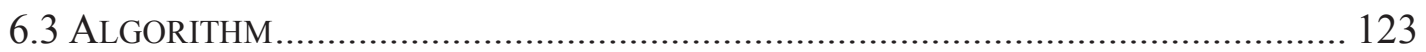

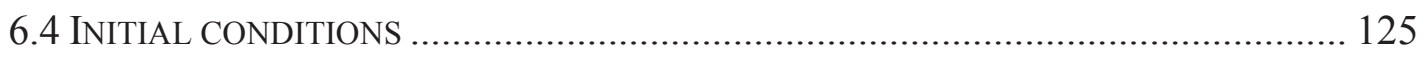

6.5 EQUILIBRIUM ENSEMBLE SYSTEM CONTROL METHOD ................................. 125

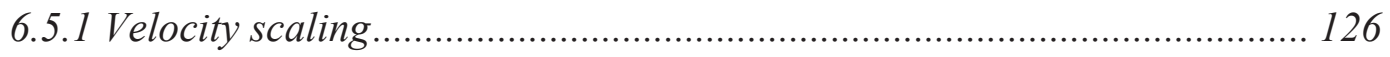

6.5.2 Berenson thermal bath..................................................................... 127

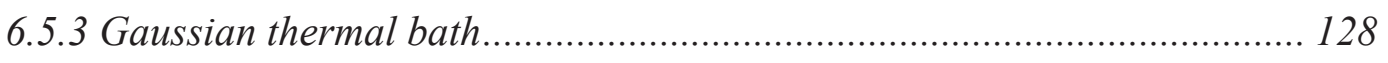

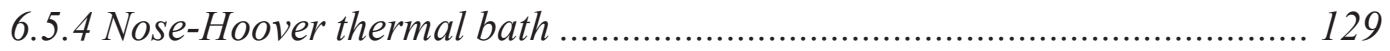

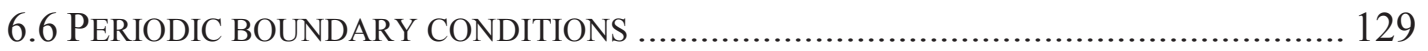

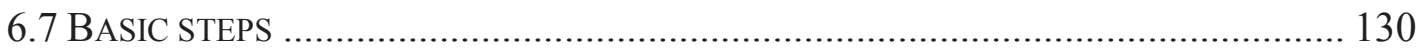

6.7.1 Build a simulation model ............................................................... 130 


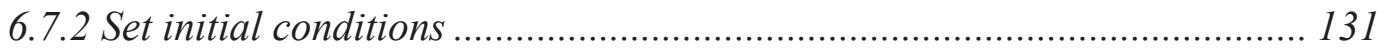

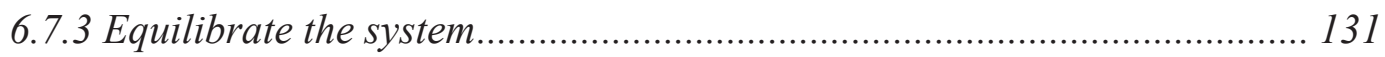

6.7.4 Determination of macroscopic quantities.............................................. 131

Interactions of Metallic Nanoparticles with Lipid Bilayers .............................. 139

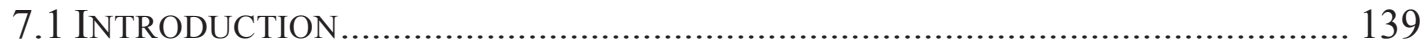

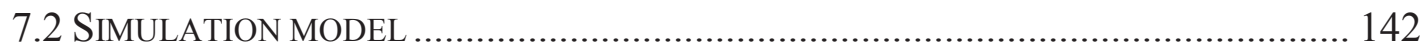

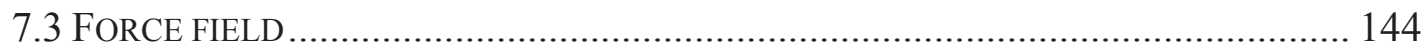

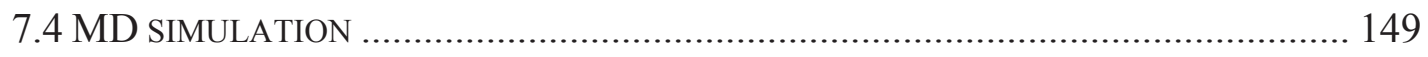

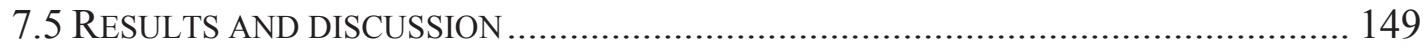

7.5.1 Permeation of AuNPs into Lipid Bilayers................................................ 149

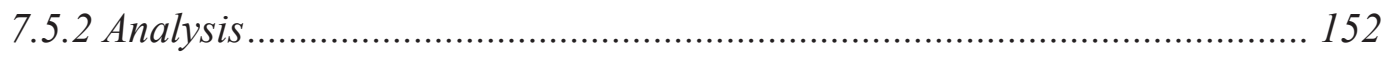

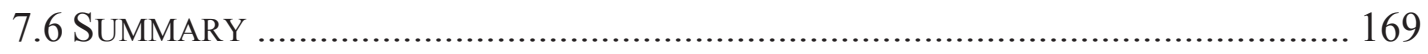

Summary and Future Work........................................................................................ 177

The List of the Selected Publications................................................................................ 181 


\section{List of figures}

Figure 2.1: Oxygen $2 p$ radial wave function (solid line), norm-conserving pseudo-wave function (dotted line), ultra-soft pseudo-wave function (dash line). 30

Figure 2.2: Illustration of Born-von Karman one-dimensional chain ring boundary conditions. 35

Figure 3.1: A ball and stick model for the DNA nucleobase (O: red, C: yellow, N: navy blue, H: blue). 46

Figure 3.3 Schematic illustration of the direction of the cluster to approach the dopamine molecule (O: red, C: yellow, N: navy blue, H: blue, X: position of QDs) 48

Figure 3.4: The energy surfaces describing interaction of the $\mathrm{Cd}_{4} \mathrm{Se}_{4}$ cluster with a dopamine molecule from different approaching directions towards the $\mathrm{O}$ site, the $\mathrm{N}$ site and the top site. 49

Figure 3.5: The energy surfaces describing interaction of the $\mathrm{Cd}_{4} \mathrm{~S}_{4}$ cluster with a dopamine molecule from different approaching directions towards the $\mathrm{O}$ site, the $\mathrm{N}$ site and the top site. 50

Figure 3.6: The charge density of the QDs-molecule complex showing the interaction from different sites: (a) O-site (b) N-site (c) Top-site. The contour density for (a) and (b) is $0.30 \mathrm{e} / \AA^{3}$. 52

Figure 3.7: QD approaching Adenine and the energy ................................................... 53

Figure 3.8: QD approaching Cytosine and the energy surface ........................................ 53

Figure 3.9: QD approaching Guanine and energy surface............................................. 54 
Figure 3.10: QD approaching Thymine and The energy surfaces

Figure 4.1: The molecular configurations and crystal structures of DMPC and DMPE.

(Atomic symbols: C-(small) yellow, O-red, P-(large) yellow, N-(large) dark blue and H(small) light blue.) 68

Figure 4.2: The triclinic unit cell of DMPC. Atomic symbols: C-(small) yellow, O-red, P(large) yellow, $\mathrm{N}$-(large) dark blue and $\mathrm{H}-($ small) light blue...... 68

Figure 4.3: The hydrophilic heads of DMPC and DMPE. Atomic symbols: C-green, O-

red, P-yellow, N-dark blue and H-(small) dark yellow.

Figure 4.4: The electron density contour plots on the plane containing the hydrophilic head-groups of DMPC and DMPE.

Figure 5.1: A schematic illustration of the conformations of the cyclo (S-Met-S-Met) molecule

Figure 5.2: Step I: Packing orientation preference of cyclo (S-Met-S-Met) where the dihedral angle of the ring with respect to the $a b$ plane of the unit cell of the crystal...... 94 Figure 5.3: Step II- Configurations of cyclo (S-Met-S-Met) crystals having different conformations of their two $\left(-\mathrm{CH}_{2}-\mathrm{CH}_{2}-\mathrm{S}-\mathrm{CH}_{3}\right)$ groups with $\phi_{\text {ring } \_ \text {ab }}=45^{\circ}$. 96 Figure 5.4: Step III- (a) Newman projection representation of the ethane molecule, (b)-(e) the flow chart showing the generation of the cyclo (S-Met-S-Met) conformers via rotation of the side chains. Atomic symbols: $\mathrm{C}$ in green, $\mathrm{N}$ in brown, $\mathrm{O}$ in red, $\mathrm{S}$ in golden yellow and $\mathrm{H}$ in blue 99

Figure 5.5: Step III- Refinement of the packing conformations of the cyclo (S-Met-SMet) crystal. 101 
Figure 5.6: The molecular and crystalline structures of the cyclo (R-Met-R-Met). Atomic symbols: $\mathrm{C}$ in yellow, $\mathrm{N}$ in navy blue, $\mathrm{O}$ in red, $\mathrm{S}$ in gray and $\mathrm{H}$ in light blue.

Figure 5.7: The ground state conformations of cyclo (S-Met-S-Met) and (R-Met-R-Met)

crystals. 108

Figure 5.8: The calculated band structures and electronic density of states for the cyclo (S-Met-S-Met) and (R-Met-R-Met) crystals. Zero of the energy is aligned to top of the valence band. 109

Figure 6.1: Geometry of a simple chain molecule illustrating the bond distance $R_{45}$, bond angel $\theta_{123}$, and torsion angle $\varphi_{2145}$

Figure 6.2: A ball and stick model of a molecule showing (a) torsion type, and (b)

inversion type bond connections.

Figure 6.3 Illustration of the periodic boundary conditions for a particle passes through

the boundary of a supercell in a MD simulation. 130

Figure 7.1: The initial simulation system of AuNPs-SLB in water. (For clarity, water molecules are not shown. The snapshot is rendered by VMD). 143

Figure 7.2: Illustration of the active chemical functional groups of a DMPC molecule and the cluster model considered for nanoparticles. 145

Figure 7.3: $\mathrm{Au}_{13}$ Cluster interacting with active sites of a DMPC molecule. 146 Figure 7.4: Simulated permeation process of AuNPs through a lipid membrane: (a) side and (b) top views of the initial position of a AuNP near the surface of a lipid bilayer; (c) side and (d) top views of the position of a AuNP after 1 ps; (e) side (f) top views of the position of a AuNP after 25 ps. 152 
Figure 7.5: Total energy variation as a function of the simulation time for AuNPs...... 152 Figure 7.6: Temperature fluctuation as a function of the simulation time for AuNPs. .. 153 Figure 7.7: Total energy variation as a function of the simulation time for AgNPs...... 153 Figure 7.8 Temperature fluctuations as a function of the simulation time for AgNPs... 154 Figure 7.9: A plot of the RMSD values of the DMPC molecules in the membrane

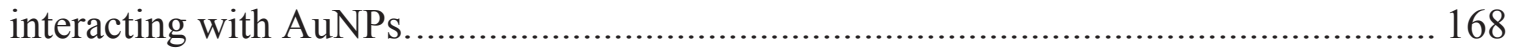
Figure 7.10: A plot of the RMSD values of the DMPC molecules in the membrane

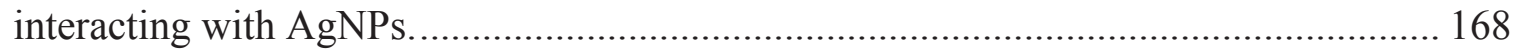
Figure 8.1: Summary of completed work and future directions. .............................. 178 


\section{List of tables}

Table 3.1: The optimized geometrical parameters of Cd4Se4/ Cd4S4 small QDs.

Table 3.2: Binding energy $\left(E_{B}\right)$ and equilibrium distance $\left(R_{B}\right)$ of the QDs-molecule

complex.

Table 3.3: Binding energy $\left(E_{B}\right)$ and equilibrium distance $\left(R_{B}\right)$ of the cluster-DNA-base

complex. 55

Table 4.1: The calculated lattice parameters of the DMPC and DMPE crystals. 69

Table 4.2: The calculated bond lengths of the hydrophilic heads of DMPC and DMPE as

shown in Figure 4.3

Table 4.3: The calculated components of dipole moments and the dipole energy per monolayer of DMPC and DMPE.

Table 4.4: Bader charge (e) of DMPC and DMPE calculated under dry and hydrated conditions. 78

Table 5.1: Step I: Choice of $\phi_{\text {ring_ab }}$ for cyclo (S-Met-S-Met). 93

Table 5.2: Step II: The optimized configurations of cyclo (S-Met-S-Met) crystals having different conformations of their two $\left(-\mathrm{CH}_{2}-\mathrm{CH}_{2}-\mathrm{S}-\mathrm{CH}_{3}\right)$ groups with $\phi_{\text {ring } \_ \text {ab }}$ of $45^{\circ} \ldots . . .95$ Table 5.3: The structural parameters of the conformers of the (S-Met-S-Met) crystal.... 99 Table 5.4: Comparison of calculated and experimental values of structural properties of the cyclo (S-Met-S-Met) and its enantiomer cyclo (R-Met-R-Met) crystals. 101

Table 5.5: Comparison of bond lengths ( $\AA$ ) for the cyclo (S-Met-S-Met) crystal 104

Table 5.6: Comparison of bond angles $\left({ }^{\circ}\right)$ for the cyclo (S-Met-S-Met) crystal. 105 
Table 5.7: Comparison of torsion angles $\left({ }^{\circ}\right)$ for the cyclo (S-Met-S-Met) crystal ......... 106 Table 7.1: Force field parameters describing the interaction of the AuNPs with DMPC.

Table 7.2: Force field parameters describing the interaction of the AgNPs with DMPC.

Table 7.3: Computed RMSD values for each DMPC molecule in the top monolayer (only this layer is considered to interact with AuNPs).

Table 7.4: Computed RMSD values for each DMPC molecule in the top monolayer (only this layer is considered to interact with AgNPs). 164 


\section{Acknowledgments}

I would not have completed my study in this Ph.D. program without the enthusiastic help from many people.

Firstly I would like to thank Prof. Ravindra Pandey, my advisor, who has provided me with this precious opportunity to continue to study in physics. He has been a great tutor for both my academic and personal life. He has taught me to learn, understand and appreciate physics from every perspective.

I would sincerely thank Prof. Donald R. Beck, Prof. Max Seel and Prof. Loredana Valenzano for being my advisory committee members and reading through this dissertation and giving valuable comments and suggestions.

I would also like to special thanks to my colleagues in our research group: Dr. Ralph Scheicher, Dr. S. Gowtham, Dr. Kah Chun Lau, Dr. Xiaoliang Zhong and Dr. Saikat Mukhopadhyay. I learned from Dr. Ralph Scheicher to use the VASP software; Dr. S. Gowtham has been very supportive in managing our computer clusters and taught me tricks about computers; Dr. Xiaoliang Zhong helped me to study the Siesta program, to name just a few. Besides, I also owe thanks to Dr. Dongwei Xu and Shu Gao for helping with matlab scripts for data analysis.

I would like to thank all of professors, staffs and my friends from physics and other department at Michigan Tech during my stay in Houghton. 
This work could not be possible without the support of my family. Special thanks to my wife, Dr. Haiying He who has always been working with me, such as revising the papers, giving suggestions about research projects and supporting me to write this dissertation. My children probably could not understand now how important they are a motivation to me, even though dragging my legs sometimes.

On the personal aspect, I would sincerely thank my mother. She has taken a lot of efforts and even sacrificed a lot in order for me to achieve higher education. In order to complete my Ph.D. study, I cannot live close to my mother and look after her. I would specially dedicate this work to my lovely mother. 


\begin{abstract}
Many types of materials at nanoscale are currently being used in everyday life. The production and use of such products based on engineered nanomaterials have raised concerns of the possible risks and hazards associated with these nanomaterials. In order to evaluate and gain a better understanding of their effects on living organisms, we have performed first-principles quantum mechanical calculations and molecular dynamics simulations. Specifically, we will investigate the interaction of nanomaterials including semiconducting quantum dots and metallic nanoparticles with various biological molecules, such as dopamine, DNA nucleobases and lipid membranes.
\end{abstract}

Firstly, interactions of semiconducting $\mathrm{CdSe} / \mathrm{CdS}$ quantum dots (QDs) with the dopamine and the DNA nucleobase molecules are investigated using similar quantum mechanical approach to the one used for the metallic nanoparticles. A variety of interaction sites are explored. Our results show that small-sized $\mathrm{Cd}_{4} \mathrm{Se}_{4}$ and $\mathrm{Cd}_{4} \mathrm{~S}_{4} \mathrm{QDs}$ interact strongly with the DNA nucleobase if a DNA nucleobase has the amide or hydroxyl chemical group. These results indicate that these QDs are suitable for detecting subcellular structures, as also reported by experiments.

The next two chapters describe a preparation required for the simulation of nanoparticles interacting with membranes leading to accurate structure models for the membranes. We develop a method for the molecular crystalline structure prediction of 1,2-Dimyristoyl-sn-glycero-3-phosphorylcholine (DMPC), 1,2-Dimyristoyl-sn-glycero-3- 
phosphorylethanolamine (DMPE) and cyclic di-amino acid peptide using first-principles methods. Since an accurate determination of the structure of an organic crystal is usually an extremely difficult task due to availability of the large number of its conformers, we propose a new computational scheme by applying knowledge of symmetry, structural chemistry and chemical bonding to reduce the sampling size of the conformation space.

The interaction of metal nanoparticles with cell membranes is finally carried out by molecular dynamics simulations, and the results are reported in the last chapter. A new force field is developed which accurately describes the interaction forces between the clusters representing small-sized metal nanoparticles and the lipid bilayer molecules. The permeation of nanoparticles into the cell membrane is analyzed together with the RMSD values of the membrane modeled by a lipid bilayer. The simulation results suggest that the AgNPs could cause the same amount of deformation as the AuNPs for the dysfunction of the membrane. 


\section{Chapter 1}

\section{Introduction}

The development of modern science and technology falls into two extremely opposite directions. On the one hand, engineers try to construct big, not small things. This is the era of gigantic trains carrying thousands of people quickly and conveniently around the world, big airplanes allowing people to fly in the sky and spaceships sending human beings into outer space. More and more skyscrapers were built in the major cities around the world. The world largest productions, such as the aircraft carriers, oil tankers, bridges, highways and power plants are all produced in this era. On the other hand, the electronic industry begins significant efforts to make things smaller. The campaign of electronics miniaturization continues from the invention of the first transistor in 1947 and the first Integrated Circuit (IC) in 1959. As the electronics together with many other areas are striving to make smaller devices and materials with smaller dimensions, engineers and scientists must turn their focus to even smaller things - nano-scale devices, and develop the new research field of nanotechnology. 
Although the terminology of nanotechnology comes in the 1980s, this new field of research had actually been predicted much earlier. It was R. P. Feynman who first proposed the idea of building machines and mechanical devices based on individual atoms in 1959. In his lecture to the American Physical Society titled "There's Plenty of Room at the Bottom", he clearly stated that the manipulation of things at the atomic level is the essence of nanotechnology. Today, after more than half of a century's development in nanotechnology, scientists and engineers are able to manipulate individual atoms and molecules, and to construct nanostructures. This makes it possible to build tiny machines smaller than the size of cells with dimensions of the order of a few nanometers.

The discovery and design of nanostructured materials are indispensable in the advances of nano devices or machines. The engineered nanomaterials possess the unique chemical and physical properties such as chemical reactivity, thermal and electrical conductivity and optical sensitivity owing to the size confinement and the high specific surface area. Nowadays, nanomaterials have been widely applied to many fields. One important application is for nanomedicine. Nanomedicine ranges from the medical applications of nanomaterials to the treatment of diseases via building nanoscale electronic devices. Nanoelectronic biosensors can often be used for diagnosis of diseases. Therefore, as a new burgeoning field, nanobiotechnology merges biological research with various fields of nanotechnology. It usually involves applying nanotools to relevant medical/biological problems and refining these applications. 
Like any other new technology, nanotechnology soon found its application in commercial products in 2000 . For instance, titanium dioxide and zinc oxide nanoparticles are used in sunscreen, cosmetics and some food products; silver nanoparticles in food packaging, clothing, disinfectants and household appliances; carbon nanotubes for stainresistant textiles; and cerium oxide as a fuel catalyst ${ }^{[1,2]}$.

It is not until recently that concern has been raised about the possible health and environment hazards of this unique group of materials - nanomaterials, in spite of the advantages that they may offer. The same physical and chemical properties of these nanomaterials which provide the sensitivity and reactivity may seriously affect the biological function of the cells of a living body. Unfortunately we are confronted with the vexatious reality that many of these applications may also bring a downside of possible risks and hazards associated with these products ${ }^{[3,4]}$. Therefore, in parallel with the development of new nanotechnologies, it is highly desirable to investigate their impact on the environment ${ }^{[5-7]}$ and issues related to workplace safety ${ }^{[8]}$, the toxicology of nanomaterials.

In this dissertation, my focus is on the computational study of interactions of nanomaterials with biological molecules using a combination of first-principles and classical molecular dynamics approaches. The goal is to investigate the toxicity of several selected nanomaterials and provide a valid toxicity evaluation scheme that may be applied to other systems as well. The experimental results show that some nanomaterials have the potential to damage skin, brain and lung tissue, and accumulate in the body ${ }^{[9]}$. 
However, besides the phenomenological observation, little is known about the underlying toxic mechanism, especially at the atomic scale. In addition, contradictory or complications of results are also found in experimental reports. For example, the study of single-walled carbon nanotubes on mitochondria in human A549 lung cells shows that the signs of cytotoxicity appeared in approximately $50 \%$ of the cells when MTT (a salt that is not soluble in water) was used, but did not get any sign of cytotoxicity when WST1 (a salt that is soluble in water) was used. Similarly no cytotoxicity was observed under the salt INT, the dye TMRE or the antibody Annexin-V environment ${ }^{[10]}$. Some experimental results even found that functionalization of carbon nanotubes can reduce toxicity $^{[11]}$.

It is apparent that the pure experimental approach has severe limitations. The complexity has also come from the fact the properties of nanomaterials can be affected by a wide range of physical parameters such as the size, shape, chemical composition or degree of agglomeration. Some of them may not be well quantified and controlled in the experiments. Heterogeneity may be another issue. All of these factors can modify the experimental results. Furthermore, as a general approach in experiments, the toxicity is measured by the number of dead or dysfunctional cells. This may be affected by multiple factors involving comprehensive interactions. Therefore, it is desirable to find a new strategy to decouple all these complexity and explain the toxicity of nanomaterials in a more definitive fashion. 
Computational modeling and simulation, on the other hand, can compensate for some of the experimental limitations. Unlike the experimental work, in a computational study, one can control each of these critical parameters independently, and identify the underlying mechanisms responsible for the experimental observation in a systematic way. Furthermore, it is also possible to simulate interactions under conditions that are inaccessible in experiments, such as extreme temperatures and pressures, strong electric or magnetic fields. Last, but not the least, computational study provides a fast and costefficient way to predict the potential hazards before something is put into the market.

The computational methods employed in this thesis work include both first-principles and atomistic methods. In general, first-principles methods can provide an accurate description of the toxicity effects of nanomaterials, but they require much more computational resources. They also have limitations including the use of the static lattice approximation mimicking absolute zero temperature conditions. On the other hand, atomistic simulation methods such as classical molecular dynamics can make up for the limitations of first principles method, but at a reduced level of accuracy. A combination of approaches based on both first-principles and molecular dynamics can therefore be used for the best balance of computational resources and the level of accuracy. Such an approach is employed to describe interactions of nanomaterials with biological molecules, which are the governing factors in predicting the toxicity of nanomaterials.

In this dissertation, several case studies are reported for the interaction of nanomaterials with biological molecules. The contents are arranged as follows. The first 
part contains first-principles based studies, while the second part presents studies based on molecular dynamics simulations. More specifically, Chapter 2 provides a detailed theoretical description of first-principles methods. Chapter 3 reports the results of firstprinciples study of $\mathrm{Mn}, \mathrm{Al}$ and $\mathrm{Ag}$ nanoparticles interacting with a biologically active molecule dopamine. Semiconductor quantum dots have applications in imaging cellular objects. Their interactions with the biological macromolecules such as DNA are studied in Chapter 4. A cell membrane is a thin, film-like structure and acts as a selective barrier allowing some special material to pass through. It is like the security door to protect the cell for its safety and has the great significance for the maintenance of the cell function. Taking into account that over $70 \%$ of biomolecules in the cell membrane belong to phospholipid molecules, structure of the phospholipid bilayer is obtained in Chapter 5 via first-principles approach and periodic boundary conditions. In Chapter 6, the newly developed computational scheme to predict molecular crystal structures from firstprinciples is tested on a cyclic di-amino acid peptide yielding good agreement with experimental data that are available. The first chapter in the second part, Chapter 7 provides a detailed description of the classical molecular dynamics (MD) approach and the core of its force fields. Lastly, in Chapter 8, a MD study of interactions of metal nanoparticles with membranes is reported. Despite the fact that the interactions of biological systems with many carbon-based nanomaterials, such as the carbon nanotubes and fullerene have been reported, there is a scarcity of the interaction with metallic nanoparticles mainly owing to the lack of force field to describe the interaction between metal nanoparticles and the lipid molecules. For this reason, a new force field is 
developed in this study based on first-principles results. For the first time we have successfully applied the molecular dynamics simulation to simulate the interaction of small metal clusters with a model lipid membrane. 


\section{References}

[1] "Nanotechnology Information Center: Properties, Applications, Research, and Safety Guidelines". American Elements. Retrieved 13 May 2011.

[2] "Analysis: This is the first publicly available on-line inventory of nanotechnologybased consumer products". The Project on Emerging Nanotechnologies. Retrieved 13 May 2011.

[3] Nel, A., Xia, T., Mädler, L. \& Li, N. Science 311, (2006) 622-627.

[4] Barnard, A. S. Nature Mater. 5, (2006) 245-248.

[5] Donaldson, K., Stone, V., Tran, C. L., Kreyling, W. \& Borm, P. J. A.Occup. Environ. Med. 61, (2004) 727-728.

[6] Lam, C. W., James, J. T., McCluskey, R., Arepalli, S. \& Hunter, R. L. Crit. Rev. Toxicol. 36, (2006) 189-217.

[7] Hyung, H., Fortner, J. D., Hughes, J. B. \& Kim, J.-H. Environ. Sci. Technol. 41, (2007) 179-184.

[8] Donaldson, K. et al. Toxicol. Sci. 92, (2006) 5-22.

[9] Hoet, P. H. M., Brüske-Hohlfeld, I. \& Salata, O. V. J. Nanobiotech. 2, (2004) $12-$ 18. 
[10] Wörle-Knirsch, J. M., Pulskamp, K. \& Krug, H. F. Nano Lett. 6, (2006) 12611268. [11] Sayes, C. M. et al. Toxicol. Lett. 161, (2006) 135-142. 


\section{Chapter 2}

\section{Electronic Structure}

Previously, physicists who are interested in the phenomenological theory to explain the experimental results are limited to the so-called primitive computational facilities. In the last decade, the development in computer technology together with the advancement in numerical algorithms has made it possible to use the basic principles of quantum mechanics to calculate the physical and chemical properties of a given system. This progress has made the computational modeling to be an important part of scientific investigations involving theory and experiments.

\subsection{Schrödinger Equation and Born-Oppenheimer Approximation}

$A b$ initio method refers to the method from first principles in which the properties of a system are obtained using the Schrödinger equation ${ }^{[1]}$. It is generally accepted that the first calculation based on the Schrödinger equation was performed by Heitler and London

on the hydrogen $\left(\mathrm{H}_{2}\right)$ molecule in $1927^{[2]}$. The time-independent Schrödinger equation can be written as 


$$
\widehat{H} \psi=i h \frac{\partial \psi}{\partial t}
$$

The corresponding Hamiltonian is:

$$
H=-\sum_{i} \frac{1}{2} \nabla_{i}^{2}-\sum_{k} \frac{1}{2 M_{k}} \nabla_{k}^{2}-\sum_{i} \sum_{k} \frac{Z_{k}}{r_{i k}}+\sum_{i<j} \frac{1}{r_{i j}}+\sum_{k<l} \frac{Z_{k} Z_{l}}{r_{k l}}
$$

Where the first term is the sum of kinetic energy of the $i^{\text {th }}$ electron; the second term is the sum of kinetic energy of the $k^{\text {th }}$ nucleus; the third term is the sum of Coulomb interaction of the $i^{\text {th }}$ electron and the $k^{\text {th }}$ nucleus; the fourth term is the sum of Coulomb interaction of the $i^{\text {th }}$ electron and the $j^{\text {th }}$ electron; the fifth term is the sum of Coulomb interaction of the $k^{\text {th }}$ nucleus and the $t^{\text {th }}$ nucleus. The expression is written here in dimensionless form or atomic units.

A complete description of a system requires a solution of the Schrödinger equation which includes both electronic and nuclear degrees of freedom. In practice, solution of the equation (2.2) is a formidable computational task, especially for systems consisting of aggregates of atoms and more than one electronic state.

Since nuclei are heavier than electrons, we can separate the electronic and nuclear coordinates in equation (2.2). This is called as the Born-Oppenheimer Approximation ${ }^{[3]}$. The wave function is separated into the nuclear wave function and the electronic wave function. The electronic Schrödinger equation can be written as: 
with

$$
\begin{gathered}
\hat{H}_{e} \psi(R, r)=E_{e} \psi(R, r) \\
\hat{H}_{e}=-\sum_{i} \frac{1}{2} \nabla_{i}^{2}-\sum_{i} \sum_{k} \frac{Z_{k}}{r_{i k}}+\sum_{i<j} \frac{1}{r_{i j}}
\end{gathered}
$$

In order to further simplify the method to solve the Schrödinger equation, one can assume that there is no interaction between the electrons of the system. Thus, the system Hamiltonian simplifies to:

$$
\widehat{H}=\sum_{i} \widehat{h}_{l} \text { with } h_{i}=-\frac{1}{2} \nabla_{i}^{2}-\sum_{k} \frac{z_{k}}{r_{i, k}}
$$

If the system falls into the weakly coupling regime of the electron correlation, the many-body problem can be mapped into a single-particle problem under an effective 'mean field' theory. Among the mean-field theoretical methods, the most popular method is based on Hartree-Fock (HF) theory ${ }^{[4,5]}$ which uses the independent electron approximation within the self-consistent field. The total wave function of an $N$-electron system is constructed based on the assumption of independent moving electron. Owing to Pauli Exclusion Principle, the wave function can be written through the Slater determinant of $N$ orthonormal single electron wave functions:

$$
\begin{aligned}
& \psi_{S D}=\frac{1}{\sqrt{N !}}\left|\begin{array}{cccc}
\chi_{1}(1) & \chi_{2}(1) & \cdots & \chi_{N}(1) \\
\chi_{1}(2) & \chi_{2}(2) & \cdots & \chi_{N}(2) \\
\vdots & \vdots & \ddots & \vdots \\
\chi_{1}(N) & \chi_{2}(N) & \cdots & \chi_{N}(N)
\end{array}\right| \\
& =\left|\chi_{1} \chi_{2} \chi_{3} \cdots \chi_{N}\right\rangle
\end{aligned}
$$


One can apply the Roothaan-Hall approximation for $\varphi$, and vary the coefficient $c_{v i}$ (e. $\mid x>$ expanded in the fixed basis set $\mid q_{i}>$ ) to minimize the integral using the energy variational principle. It leads to the determinant equation (2.6):

$$
\begin{gathered}
\sum_{i=1}^{N}\left(F_{\mu \nu}-\varepsilon_{i} S_{\mu \nu}\right) c_{v i}=0 \\
\left|\begin{array}{cccc}
F_{11}-E S_{11} & F_{12}-E S_{12} & \cdots & F_{1 N}-E S_{1 N} \\
F_{21}-E S_{21} & F_{22}-E S_{22} & \cdots & F_{2 N}-E S_{2 N} \\
\vdots & \vdots & \ddots & \vdots \\
F_{N 1}-E S_{N 1} & F_{N 2}-E S_{N 2} & \cdots & F_{N N}-E S_{N N}
\end{array}\right|=0
\end{gathered}
$$

$F_{\mu \nu}$ is:

$$
F_{\mu \nu}=\left\langle\mu\left|-\frac{1}{2} \nabla^{2}\right| v\right\rangle-\sum_{k} Z_{k}\left\langle\mu\left|\frac{1}{r_{k}}\right| v\right\rangle+\sum_{\lambda \sigma} 2 \sum_{i=1}^{\text {occupied }} c_{\lambda i}^{*} c_{c i}\left[\left(\mu \nu\left|\frac{1}{r_{i j}}\right| \lambda \sigma\right)-\frac{1}{2}\left(\mu \lambda\left|\frac{1}{r_{i j}}\right| v \sigma\right)\right]
$$

The HF method uses a single electron wave function $x$ which assumes an electron moves independently in the average potential field. Therefore, one cannot consider the role of instantaneous correlation between the electrons - the electron correlation. On the other hand, the Slater determinant wave function used by the HF method satisfies the Pauli Exclusion Principle, and this part of the electron correlation is referred to as the exchange interaction. In general, the electron correlation energy is defined as the difference between the 'true' ground state energy and the HF energy $\left(E_{c o r r}=E-E_{H F}\right)$. The contribution of the electron correlation energy to the total energy of the system is likely 
to be small, only 0.3 to $2 \%$. But, it is very important in determining accurate chemical reactions or electronic excitations. It is generally calculated by the Møller-Plesset perturbation theory ${ }^{[6,7]}$, Coupled-cluster theory $(\mathrm{CC})^{[8.9]}$ or other methods.

\subsection{Density functional theory}

The basic idea of the density functional theory (DFT) comes from Thomas and Dirac as early as the 1930s. DFT provides a first-principles computational framework; it can solve many of the problems in atoms, molecules, and solids such as the calculation of the ionization potential, vibrational spectra, and the choice of the catalytically active sites, biological molecules electronic structure, and electronic band structure. Note that Kohn and Pople received the Nobel Prize in Chemistry in 1998 owing to their pioneering work in DFT.

For the $N$-electron system, we can use $\varphi\left(x_{1}, x_{2}, \cdots x_{N}\right)$ to represent the function of the state in the available $4 N$-dimensional space as the $N$ particles are in three-dimensional space and spin space. According to the physical meaning of the wave function, we have:

$$
\rho\left(x_{1}, x_{2}, \cdots\right.
$$

Here $\rho=\Delta N / l^{3}$ is the density of electrons; $d \vec{\imath}=u x_{1} u x_{2} \cdots \quad$ represents a small volume element of $4 N$-dimensional space. $\rho\left(x_{1}, x_{2}, \cdots \quad\right.$ is the probability of 
occurrence of an electron in a small volume element of the $4 \mathrm{~N}$-dimensional space. The $\mathrm{N}$ electron system wave function in the coordinate space can be written as:

$$
\left|\varphi_{i}\left(x^{N}\right)\right\rangle=\left\langle x_{1}, x_{2}, \cdots\right.
$$

\subsubsection{Thomas-Fermi theory and related models}

In a Thomas and Fermi model ${ }^{[10,11]}$, the energy of an $N$-electron system can be expressed as:

$$
E[\rho]=T[\rho]+\int \rho(r) v(r) d r+V_{e e}[\rho]
$$

$T[\rho]$ is the kinetic energy of the electrons, the second term is the nuclear and electronic interaction potential energy; $V_{e e}[\rho]$ is Coulomb interaction energy. The total kinetic energy is:

$$
T[\rho]=C_{F} \int \rho^{5 / 3}(r) d r \text { and } C_{F}=\frac{3}{10}\left(3 \pi^{2}\right)^{2 / 3}=2.871
$$

The total energy can then be written as:

$$
E_{T F}[\rho]=C_{F} \int \rho^{5 / 3}(r) d r-Z \int \frac{\rho(r)}{r} d r+\frac{1}{2} \iint \frac{\rho\left(r_{1}\right) \rho\left(r_{2}\right)}{\left|r_{1}-r_{2}\right|} d r_{1} d r_{2}
$$


Later, Dirac added the electronic exchange energy in $V_{e e}[\rho]$ of the Thomas-Fermi model. The electronic system is still a free particle in a box with the boundary conditions $\varphi(x+l)=\varphi(x)$. The independent electronic wave function is:

$$
\varphi\left(\kappa_{x}, \kappa_{y}, \kappa_{z}\right)=\frac{1}{l^{3 / 2}} e^{i\left(K_{x} x+K_{y} y+K_{z} z\right)}=\frac{1}{V^{1 / 2}} e^{i k r}
$$

And the first-order reduced density matrix is:

$$
\rho\left(r_{1}, r_{2}\right)=\frac{2}{V} \sum e^{i k\left(r_{1}-r_{2}\right)}=\frac{1}{4 \pi} \int e^{i k\left(r_{1}-r_{2}\right)} d k=\frac{1}{4 \pi} \int_{0}^{k_{f}} k^{2} d k \iint e^{i k r_{12}} \sin \theta d \theta d \varphi
$$

Quantum number $k_{f}$ is a function of position, corresponding to the Fermi level,

$$
k_{f}=[3 \pi \rho(r)]^{1 / 3}
$$

Let $r=\frac{1}{2}\left(r_{1}+r_{2}\right), s=\frac{1}{2}\left(r_{1}-r_{2}\right), t=k_{f} s$, We can then rewrite equation (2.14):

$$
\begin{aligned}
& \rho_{1}\left(r_{1}, r_{2}\right)=\frac{1}{4 \pi^{3}} \int_{0}^{k_{f}} k^{2} d k \int_{0}^{\pi} \sin \theta e^{i k r} d \theta \int_{0}^{2 \pi} d \phi \\
& =3 \rho(r)\left[\frac{\sin t-t \cos t}{t^{3}}\right]=\rho_{1}(r, s)
\end{aligned}
$$

The kinetic energy is: 


$$
T[\rho]=\frac{3}{10}\left(3 \pi^{2}\right)^{2 / 3} \int \rho(r)^{5 / 3} d r=C_{F} \int \rho(r)^{5 / 3} d r
$$

Here $C_{F}=2.8712$, which is same as obtained in the Thomas-Fermi model.

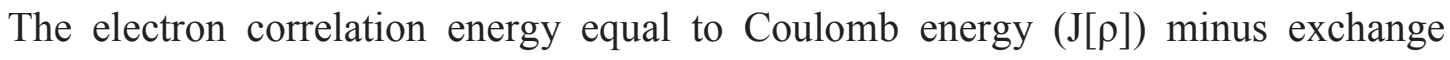
energy $(K[\rho])$ :

$$
\begin{gathered}
V_{e e}[\rho]=J[\rho]-K[\rho] \text { and } \\
K[\rho]=\frac{1}{4} \iint \frac{\left[\rho_{1}(r, s)\right]^{2}}{s} d r d s=C_{x} \int \rho^{\frac{3}{4}}(r) d r
\end{gathered}
$$

Here $C_{x}=0.7386$

The total energy in the Thomas-Fermi-Dirac model defined as follows:

$$
E_{T F D}[\rho]=C_{F} \int \rho(r)^{5 / 3} d r+\int \rho(r) v(r) d r+J(\rho)-C_{x} \int \rho(r)^{4 / 3} d r
$$

Based on this method, several researchers have tried various approaches to improve the accuracy for the Thomas-Fermi-Dirac model, but the effect had been unsatisfactory, until Kohn's work appeared in 1964 providing the fundamental theorems - the ground state of Thomas-Fermi-Dirac model may be taken as an approximation to an 'exact' ground state of the system ${ }^{[12,13]}$. 


\subsubsection{Hohenberg-Kohn Theorem}

In the Thomas-Fermi and the Thomas-Fermi-Dirac models, the nature of the system depends only on the electron density. This basic assumption was proven by HohenbergKohn in two important theorems which are now regarded as cornerstones of the density functional theory (DFT).

The essential statements of DFT are:

(i): when the external potential is finalized, the electron density of the ground state should also be uniquely determined.

(ii): the calculated ground state energy with the approximated density, $\rho$ ' must not be less than the true ground state energy with density $\rho$.

The first Hohenberg-Kohn theorem tells us that the electron density of a system can only be finalized when it is in the ground state, but it does not tell us how to determine it. In fact, the wave function and the electron density of the $N$-electron system cannot be exactly solved. The second Hohenberg-Kohn theorem states that the total energy of the system depends on the electron density with functional variational characteristics, and all observables of the system can then be calculated.

Application of electron density variational leads to

$$
\delta E[\rho]=E[\rho+\delta \rho]-E[\rho]=0
$$


This is under the constraint that the number of electrons of the system is conserved:

$$
\int \rho(r) d r=N
$$

Using the Lagrange multipliers, under the constraint conditions of equation (2.21), we can take the functional variation and get:

$$
\frac{\delta\left\{E[\rho]-\mu\left[\int \rho(r) d r-N\right]\right\}}{\delta \rho}=0
$$

Now the question is about the specific expression of the energy functional form. The energy functional form can be constructed as follows:

1. The electronic kinetic energy $(T(\rho))$. The electron can be regarded as the outer potential movement in the momentum space, so the kinetic energy of the electrons can depend on the electron density.

2. The nucleus attractive electronic energy is calculated using:

$$
V_{e x t}=\int \rho(r) V_{n e}(r) d r
$$

3. The electronic Coulomb interaction energy is calculated using:

$$
J[\rho]=\frac{1}{2} \int \frac{\rho(r) \rho\left(r^{\prime}\right)}{\left|r-r^{\prime}\right|} d r d r^{\prime}=\frac{1}{2} \int V_{c}(r) \rho(r) d r
$$


4. The interaction between electrons includes electronic exchange interaction and electron correlation energy, unified and referred to as $E_{x c}[\rho]$.

The total energy is

$$
E(\rho)=T[\rho]+V_{e x t}[\rho]+J[\rho]+E_{x c}^{\prime}[\rho]
$$

Application of variational principles leads to

$$
\frac{\delta E(\rho)}{\delta \rho}=\frac{\delta T[\rho]}{\delta \rho}+\frac{\delta}{\delta \rho}\left[\int \rho(r) V_{n e}(r) d r+\frac{1}{2} \int V c(r) \rho(r) d r\right]+\frac{\delta E_{x c}^{\prime}[\rho]}{\delta \rho}
$$

the Euler-Lagrange equation is

$$
\frac{\delta T[\rho]}{\delta \rho}+V_{n e}+V_{c}+\frac{\delta E_{x c}[\rho]}{\delta \rho}=\mu
$$

Thomas-Fermi theory adopts a direct approximation (i.e. free electron gas model) ignoring the electronic exchange-correlation term in equation (2.25). On the other hand, Kohn and Sham introduced non-interaction model in which motion of electrons is seen as a stand-alone electronic external field leads to a simpler energy expression:

$$
E(\rho)=T_{0}[\rho]+\int V_{\text {eff }}(r) \rho(r) d r
$$

$T_{0}[\rho]$ is the kinetic energy of the electrons with the Euler-Lagrange equation: 


$$
\frac{\delta T_{0}[\rho]}{\delta \rho}+V_{\text {eff }}=\mu
$$

Comparison of (2.27) with (2.29) yields

$$
V_{\text {eff }}=\frac{\delta T[\rho]}{\delta \rho}-\frac{\delta T_{0}[\rho]}{\delta \rho}+V_{n e}+V_{c}+\frac{\delta E_{x c}^{\prime}[\rho]}{\delta \rho}
$$

Now, we can construct the equivalent stand-alone electronic Hamiltonian system:

$$
\hat{H}=\sum_{i}^{N}\left(-\frac{1}{2} \nabla_{i}^{2}+V_{\text {eff }}\left(r_{i}\right)\right)
$$

This operates on the ground state wave function determinant

$$
\Psi=\frac{1}{\sqrt{N !}} \operatorname{det} \mid \varphi_{1} \varphi_{2} \cdots
$$

$\varphi_{i}$ is the $\mathrm{i}^{\text {th }}$ eigenstate of the Single-electron Hamiltonian $h_{i}$, namely:

$$
\hat{h}_{i} \varphi_{i}=\left[-\frac{1}{2} \nabla_{i}^{2}+V_{\text {eff }}\left(r_{i}\right)\right] \varphi_{i}=\varepsilon_{i} \varphi_{i}
$$

For the kinetic energy, we can use this formula to calculate:

$$
T[\rho]=\left\langle\Psi\left|\sum_{i=1}^{N}\left(-\frac{1}{2} \nabla_{i}^{2}\right)\right| \Psi\right\rangle
$$

Here the Electron density is defined as: 


$$
\rho=\sum_{i=1}^{N}\left|\varphi_{i}\right|^{2}
$$

Now we can define the exchange-correlation functional as:

$$
V_{x c} \equiv \frac{\delta T[\rho]}{\delta \rho}-\frac{\delta T_{0}[\rho]}{\delta \rho}+\frac{\delta E_{x c}^{\prime}[\rho]}{\delta \rho}=\frac{\delta E_{x c}[\rho]}{\delta \rho}
$$

We bring it into (2.27) to write:

$$
V_{e f f}=V_{n e}+V_{c}+V_{x c}
$$

Thus, we have Kohn-Sham equation:

$$
\left[-\frac{1}{2} \nabla_{i}^{2}+V_{n e}\left(r_{i}\right)+V_{c}\left(r_{i}\right)+V_{x c}\left(r_{i}\right)\right] \varphi_{i}=\varepsilon_{i} \varphi_{i}
$$

The expression of the total energy is:

$$
E=\sum_{i}^{N} \varepsilon_{i}-\int V_{n e}(r) \rho(r) d r-\frac{1}{2} \int \frac{\rho(r) \rho\left(r^{\prime}\right)}{\left|r-r^{\prime}\right|} d r d r^{\prime}+E_{x c}[\rho]
$$

\subsubsection{Exchange and correlation functional forms}

In solving Kohn-Sham equations, difficulties and challenges lie in how to establish

the exchange-correlation energy. Kohn and Sham put forward the so-called local density approximation (LDA) assuming that the electron density of the system is homogeneous. 
The electron exchange-correlation energy then only depends on the density of electrons and cannot be dependent on the gradient of the electron density.

$$
E_{x c}^{L D A}[\rho]=\int \rho(r) \varepsilon_{x c}(\rho) d r
$$

Here $\varepsilon_{x c}$ is the homogeneous electron gas exchange-correlation energy for a single electron. The exchange-correlation potential can be written as:

$$
V_{x c}^{L D A}(r)=\frac{\delta E_{x c}^{L D A}}{\delta \rho(r)}=\varepsilon_{x c}[\rho(r)]+\rho(r) \frac{\partial \varepsilon_{x c}(\rho)}{\partial \rho}
$$

The Kohn-Sham equation can then be written as:

$$
\begin{gathered}
{\left[-\frac{1}{2} \nabla^{2}+V(r)+\int \frac{\rho\left(r^{\prime}\right)}{\left|r-r^{\prime}\right|} d r^{\prime}+V_{x c}^{L D A}(r)\right] \varphi_{i}=\varepsilon_{i} \varphi_{i}} \\
\text { With } \varepsilon_{x}[\rho]=\varepsilon_{x}[\rho]+\varepsilon_{c}[\rho]
\end{gathered}
$$

For the electronic exchange potential energy component, the Thomas-Fermi-Dirac model is:

$$
\varepsilon_{x}[\rho]=-C_{x} \rho(r)^{1 / 3} C_{x}=\frac{3}{4}\left(\frac{3}{\pi}\right)^{1 / 3}
$$

$\varepsilon_{c}[\rho]$ describes the Coulomb interaction of the electrons caused by their dynamic interaction. The earliest potential energy was given by EP Wigner: 


$$
\varepsilon_{c}(\rho)=-\frac{0.44}{r_{s}+7.8} \text { and } r_{s}^{3}=\frac{3}{4 \pi \rho(r)}
$$

Here $r_{s}$ is the classical radius of the electron.

Later, several forms were proposed. For example, the Vosko, Wilk and Nusair's form ${ }^{[14]}$ is:

$$
\begin{gathered}
\varepsilon_{c}(\rho)=\frac{A}{2}\left\{\ln \frac{x^{2}}{X(x)}+\frac{2 b}{Q} \tan ^{-1} \frac{Q}{2 x+b}-\frac{b x_{0}}{X\left(x_{0}\right)}\left[\ln \frac{\left(x-x_{0}\right)^{2}}{X(x)}+\frac{2\left(b+2 x_{0}\right)}{Q} \tan ^{-1} \frac{Q}{2 x+b}\right]\right\} \\
x=r_{s}^{1 / 2}, X(x)=x^{2}+b x+c, Q=\left(4 c-b^{2}\right)^{3 / 2} \\
A=0.62184, x_{0}=-0.409286, b=12.0720, c=42.7198
\end{gathered}
$$

Perdew and Zunger's ${ }^{[15]}$ form is:

$$
\varepsilon_{c}(\rho)=\left\{\begin{array}{cc}
-0.1423 /\left(1+1.9529 r_{s}^{1 / 2}+0.334 r_{s}\right) & r_{s} \geq 1 \\
-0.480+0.311 \ln r_{s}-0.0116 r_{s}+0.0020 r_{s} \ln r_{s} & r_{s}<1
\end{array}\right.
$$

The LDA approximation using the uniform electron gas model appears to be physically incorrect where a slow change in electron density is expected, e.g. ionic solid state systems. In order to improve the accuracy of the DFT method, the generalized gradient approximation (GGA) ${ }^{[16,17]}$ was developed. The GGA form can be written as

$$
E_{x c}^{G G A}[\rho]=\int \rho(r) \varepsilon_{x c}(\rho, \nabla \rho) d r
$$


Becke ${ }^{[18]}$ used the fitting method and derived:

$$
\varepsilon_{x}^{\mathrm{B} 88}=\varepsilon_{x}^{\mathrm{LDA}}\left[\frac{1+s^{*} \mathrm{a}_{1}^{*} \sinh ^{-1}\left(s^{*} \mathrm{a}_{2}\right)+\mathrm{a}_{3} * s^{2}}{1+s^{*} \mathrm{a}_{1}{ }^{*} \sinh ^{-1}\left(s^{*} \mathrm{a}_{2}\right)}\right]
$$

Perdew ${ }^{[19]}$ advocated minimizing the introduction of fitting parameters. He proposed the PBE exchange-correlation energy expression as:

$$
\varepsilon_{x}^{\mathrm{PBE} 96}=\varepsilon_{x}^{\mathrm{LDA}}\left[1+\mathrm{k}-\frac{\mathrm{k}}{1+\frac{\mathrm{u}}{\mathrm{k}} s^{2}}\right]
$$

Where $\mathrm{k}=0.804$ and $\mathrm{u}=021951$.

Recall that the HF theory contains the precise description of the exchange capacity in a many-electron system. Accordingly, in a mixing section of the DFT, HF exchange can help to improve accuracy of the functional form. The most simplified hybrid functional forms with the HF exchange terms can be written as:

$$
E_{X C}=a E_{X}^{e x z c t}+(1-a) E_{X}^{G G A}+E_{c}^{G G A}
$$

A more practical hybrid functional type is B3LYP ${ }^{[20-22]}$ :

$$
E_{x c}^{B 3 L Y P}=E_{x c}^{L D A}+a_{0}\left(E_{x}^{H F}-E_{x}^{L D A}\right)+a_{x}\left(E_{x}^{G G A}-E_{x}^{L D A}\right)+a_{c}\left(E_{c}^{G G A}-E_{c}^{L D A}\right)
$$


Where $a_{0}=0.20, a_{\mathrm{x}}=0.72$, and $a_{\mathrm{c}}=0.81 . E_{\mathrm{x}}{ }^{\mathrm{GGA}}$ and $E_{\mathrm{c}}{ }^{\mathrm{GGA}}$ are generalized gradient approximations: the Becke 88 exchange functional and the correlation functional of Lee, Yang and Parr for B3LYP, and $E_{\mathrm{c}}{ }^{\mathrm{LDA}}$ is the VWN local-density approximation to the correlation functional.

\subsection{Pseudopotential}

The electronic structure of constituting atoms of a material or molecule has two basic characteristics. The core electrons of an atom remain nearly unchanged in making transition from atomic to molecular or solid state, and the valence electrons get modified in molecular or solid state. Therefore, one can build an effective potential to represent electron density of the core electrons in electronic structure calculations, thereby reducing the computational requirements.

Norm-conserving pseudopotentials ${ }^{[23,24]}$ are a common class of pseudopotentials. They are introduced to remove the core electrons and the strong nuclear potential and act on a set of pseudo wave functions. The pseudo-wave function used to represent the core electrons is defined by a smoothly-varying nodeless function below the cut-off radius $\left(R_{\mathrm{c}}\right)$. The total charge enclosed within $R_{\mathrm{c}}$ is equal to the total charge of all-electron wave functions. Therefore, the pseudo wave function beyond $R_{\mathrm{c}}$ reproduces the all-electron wave function. The choice of the value of $R_{\mathrm{c}}$ of a pseudopotential can affect the calculation accuracy dramatically. In this way, the ferocious oscillation of electron wave function in the core is removed and transformed into a smoothly changing wave function, 
which has no nodes. Use of pseudopotentials not only reduces the number of electrons to be treated in the system, but also allows one to expand the wave function using a much smaller plane wave basis set. Since the need of solving for the core electrons is totally removed, such an approximate pseudopotential can be given in the case of a valenceelectron only solution. It should be noted, however, that the use of pseudopotentials for the first row (carbon, nitrogen, oxygen) or transition metals (nickel, copper, and palladium) elements require high plane wave cut-off energy, due to the highly localized valence electron orbitals. Norm-conserving pseudopotentials can be applied in both real space and reciprocal space; and the real space method provides better scalability for the system.

In order to construct a pseudopotential wave function, one normally needs to match the following conditions: firstly, the configured function must be smooth without any nodes. Secondly, for a typical electronic configuration, the pseudopotential eigenvalue must be equal to the actual eigenvalue. Thirdly, the actual wave function should be equal to pseudopotential wave function in the core radius, $R_{\mathrm{c}}$. Lastly, although the pseudopotential wave function is not same as the actual wave function inside the core radius, but the charge density should be equivalent inside the core radius. This is the meaning of norm-conserving. Based on the second and third point conditions, pseudopotential is equal to the genuine potential outside core radius. Therefore, the core radius is chosen based on the following considerations: big enough to make soft pseudopotential, small enough to keep good transferability, and not too small to be very 
close to the outmost radial node. Note that some of the pseudopotential programs may require additional conditions to determine the pseudopotential function. For example, the radial part of the wave function of the Troullier-Martines (TM) pseudopotential is defined as:

$$
\begin{gathered}
R_{l}^{P S}=R_{l}^{A E}(r) r \geq r_{c}^{l} \text { and } \\
R_{l}^{P S}=r^{l} e^{p(r)} r \leq r_{c}^{l} \text { with } p(r)=c_{0}+c_{2} r^{2}+c_{4} r^{4}+\cdots \cdots+c_{12} r^{12}
\end{gathered}
$$

For any reference energy, the nonlocal pseudopotential can be written as: $v_{N L}=\frac{\left|\chi_{i}\right\rangle\left\langle\chi_{i}\right|}{\left\langle\chi_{i} \mid \varphi_{i}^{p s}\right\rangle},\left|\chi_{i}\right\rangle$ is auxiliary local wave function, $\left|\chi_{i}\right\rangle=\left(\varepsilon_{i}-T-v_{l o c}\right)\left|\varphi_{i}^{p s}\right\rangle$.

We can extend to multiple reference energy defining $B_{i j}$ as:

$$
B_{i j}=\left\langle\phi_{i}|| \chi_{j}\right\rangle\left|\beta_{i}\right\rangle=\sum_{i j}\left(B_{i j}^{-1}\right)_{i j}\left|\chi_{j}\right\rangle, v_{N L}=\sum_{i j} B_{i j}\left|\beta_{i}\right\rangle\left\langle\beta_{i}\right|
$$

Under the Norm-conservation conditions, $Q_{i j}=\left\langle\varphi_{i} \mid \varphi_{j}\right\rangle_{R}-\left\langle\varphi_{i}^{p s} \mid \varphi_{j}^{p s}\right\rangle=0$, and $\mathrm{B}_{\mathrm{ij}}$ and $\mathrm{v}_{\mathrm{NL}}$ are Hermitian. 


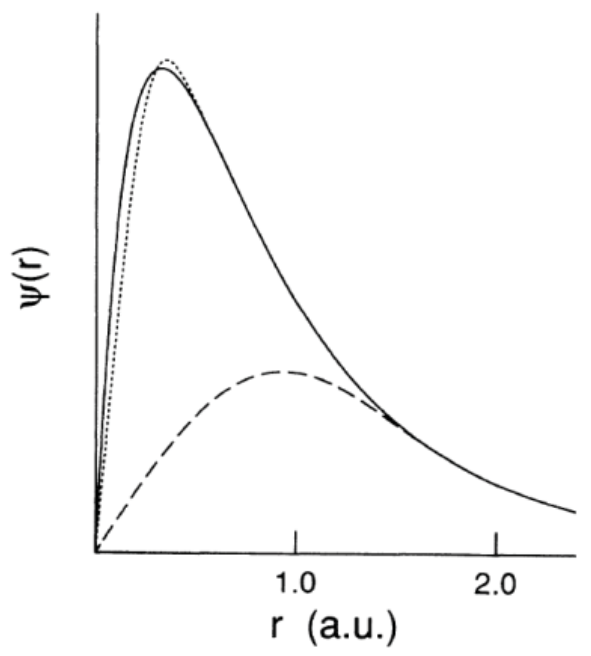

Figure 2.1: Oxygen 2p radial wave function (solid line), norm-conserving pseudo-wave function (dotted line), ultra-soft pseudo-wave function (dash line).

Because of norm-conserving condition constraints, norm-conserving pseudopotentials for the first period elements and transition metals did not significantly reduce the amount of computation. The Ultrasoft pseudopotentials which do not follow the norm-conserving pseudopotential model are proposed.

We define $\mathrm{S}$ to be:

$$
\begin{gathered}
S=1+\sum_{i j} Q_{i j}\left|\beta_{i}\right\rangle\left\langle\beta_{j}\right| \\
v_{N L}=\sum_{i j}\left(B_{i j}+\varepsilon Q_{i j}\right)\left|\beta_{i}\right\rangle\left\langle\beta_{j}\right| \\
\left\langle\varphi_{i}^{p s}|S| \varphi_{j}^{p s}\right\rangle_{R}=\left\langle\varphi_{i} \mid \varphi_{j}\right\rangle_{R}
\end{gathered}
$$

So we get: 


$$
\left(T+v_{l o c}+v_{N L}\right)\left|\varphi_{i}^{p s}\right\rangle=\varepsilon_{i} S\left|\varphi_{i}^{p s}\right\rangle
$$

Blöchl et al. ${ }^{[25]}$ proposed the Projector augmented wave (PAW)) method linking allelectronic wave functions to the pseudo wave functions using the transformation defined as:

$$
\varphi=\hat{T} \varphi^{p s}, \hat{T}=\hat{I}+\sum_{R} \hat{T}_{R}
$$

The partial wave expansion around the atom is:

$$
\begin{aligned}
& |\varphi\rangle=\left|\varphi^{p s}\right\rangle+\sum_{m} c_{m}\left\{\left|\varphi_{m}\right\rangle-\left|\varphi_{m}^{p s}\right\rangle\right\} \\
& c_{m}=\left\langle\beta_{m} \mid \varphi^{p s}\right\rangle \\
& \hat{T}=\hat{I}+\sum_{m}\left\{\left|\varphi_{m}\right\rangle-\left|\varphi_{m}^{p s}\right\rangle\right\}\left\langle\beta_{m}\right|
\end{aligned}
$$

The energy system, the charge density and other information can be obtained through the pseudo wave function.

\subsection{Basis sets}

The wave function of an electron in single-electron orbit can be expressed as a finite linear combination of analytic functions: $\varphi_{\mathrm{i}}=\sum_{\mu} \mathrm{a}_{\mu} \chi_{\mu}$, where $\chi_{\mu}$ is called a basis function and its collection $\left\{\chi_{\mu}\right\}$ is called the basis group. A common solution is to choose the basic functions of the atoms in the molecule to be the atomic orbital $(s, p, d, \ldots)$, that is, by a linear combination of atomic orbitals (LCAO) to get the molecular orbital. 
Atomic orbitals can generally be written as:

$$
\chi(r, \theta, \phi)=R_{n}(r) Y_{l m}(\theta, \phi)
$$

For $R_{n}(r)$, in general, two kinds of basic functions are used in the molecular orbital (MO) theory: Slater Type Orbitals (STOs) and Gaussian Type Orbitals (GTOs).

STO is the class of hydrogen atomic orbitals, an intuitive choice. Its expression is:

$$
\chi(\mathrm{r}, \theta, \varphi) \propto \mathrm{r}^{\mathrm{n}-1} \mathrm{e}^{-\varsigma \mathrm{r}} \mathrm{Y}_{\mathrm{lm}}
$$

Where $n$ is the principal quantum number, which is equivalent to the radial wave function of hydrogen ions with the orthogonal restriction removed. It requires a preexponential factor for a single type to simplify calculations. Slater-type basis functions are very good for small systems, but calculations of three-center and four-center twoelectron integrals become relatively difficult. In 1950, Boys proposed using Gaussian type function as a basis function to expand the molecular orbitals. Gaussian functions preceded by different factors correspond to $s, p_{x}, p_{y}, p_{z}, d_{x y}, d_{y z}, d_{z x}$ etc. Gaussian type functions can be obtained.

$$
\chi(x, y, z) \propto x^{i} y^{j} z^{k} e^{-\alpha r^{2}}
$$

The Gaussian function is an exponential function of $r^{2}$, which can be decomposed into $x^{2}+y^{2}+z^{2}$, so that one can easily separate three dimensional integral to onedimensional integrals, thus greatly simplifying electronic structure calculations. 
Beside the Gaussian-type orbital and Slater-type orbital, the plane wave basis set ${ }^{[30,31]}$ is also very popular to use in the field of Condensed Matter Physics. Any single electron wave function can also be written in the form of superposition of plane waves:

$$
\varphi_{n}(r)=\int C_{n}(g) e^{i g \cdot r} d g
$$

If $g$ is equal to 0 , the wave function is a constant.

The plane wave expansion often needs the help of discrete periodic boundary conditions (PBC). The periodic boundary conditions can be used for a crystal, while molecules (0D), wires (1D) and surfaces (2D) can be defined via supercells with imposed periodic boundary conditions.

If the Potential field of a lattice has periodicity, the wave function can be expressed as:

$$
\varphi_{n k}(r)=u_{n k}(r) e^{i k \cdot r}
$$

$u_{n k}(r)$ is a periodic function of the lattice.

According to the Bloch theorem, one-electron wave function has a periodic part which can be expressed via discrete Fourier expansion (reciprocal lattice). Thus, the plane wave can be written as:

$$
\varphi_{n k}(r)=\sum_{G} C_{n, k+G} e^{i(K+G) \cdot r}
$$


Where $G$ is an integer multiple of the reciprocal lattice vector $b . K$ is taken from the first Brillouin Zone (of the period is $2 \pi / R$ ) with $a_{i} \cdot b_{j}=2 \pi \delta_{i j}$. Calculations require truncation of the $G$ value, thus the plane wave basis set has an advantage of having increased cut-off energy to systematically improve the nature of the base set of functions.

Bloch theory can also be seen as the wave function classification at $k$ points. In general, for every $k$, the number of occupied orbitals within the unit cell is equal to the number of electrons. Therefore, we find that the charge density on an infinite number of occupied orbitals by the summation transform into Brillouin zone integration. The charge density can be calculated using this formula:

$$
\rho=\sum_{n}^{o c c} \int_{B Z} \varphi_{n k}^{*}(r) \varphi_{n k}(r) d r d k
$$

Born-von Karman boundary conditions ${ }^{[32,33]}$ allows one to further use discrete $k$. Assume that there is a large enough period of $N$, one-dimensional case can be viewed as a one-dimensional chain ring as shown in Figure 2.2. 


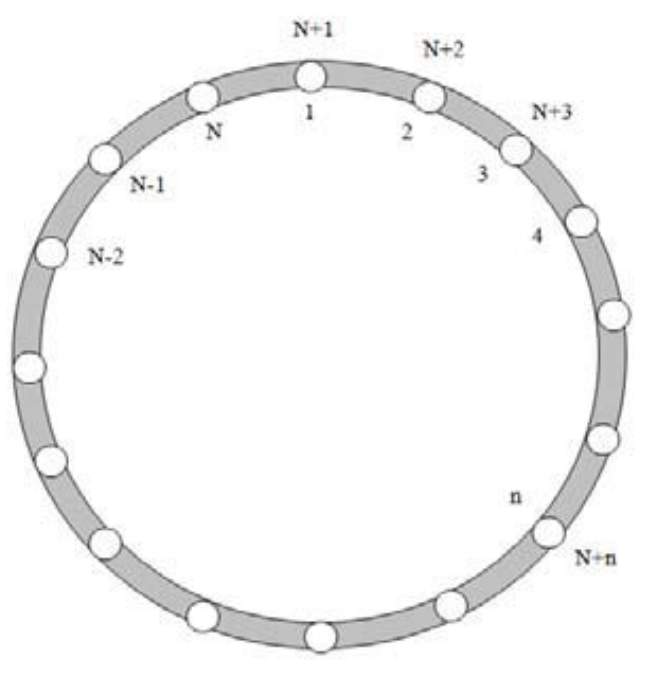

Figure 2.2: Illustration of Born-von Karman one-dimensional chain ring boundary conditions.

$$
\varphi_{n}=\varphi_{N+n} \Rightarrow e^{i k N R}=1 \Rightarrow k=\frac{2 \pi}{N R} m \text { with the integer } m \text { ranging from }-N / 2 \text { to } N / 2 \text {. Thus, }
$$

we can take discrete values of $k$ in the reciprocal space of the first Brillouin zone.

It is to be noted that calculations of the kinetic energy are much more convenient in the reciprocal space while that of the potential energy-related terms are in the real space. Therefore, calculations of the Hamiltonian matrix elements often require Fourier transform. Furthermore, the Born-von Karman boundary conditions in the real space correspond to the density of the $k$ points of the Brillouin zone in the reciprocal space. Accuracy of the Brillouin zone integration therefore becomes important in electronic structure calculations. Its general form is

$$
\sum_{n} \frac{1}{\Omega_{B Z}} \int_{\Omega_{B Z}} \varepsilon_{n k} \Theta\left(\varepsilon_{n k}-\mu\right) d k
$$


In generally, the integral can be turned into summation of $k$ points for selected points with weights:

$$
\frac{1}{\Omega_{B Z}} \int_{\Omega_{B Z}} \Rightarrow \sum_{k} w_{k}
$$

The selection of $k$ points is generally performed by the Monkhorst-Pack method which changes a three-dimensional problem into three one-dimensional problems. A set of Monkhorst-Pack grid is selected via uniform dividing rules within the lattice point sets in the Brillouin zone.

\subsection{Vienna Ab initio Simulation Package (VASP)}

$\operatorname{VASP}^{[34-37]}$ is a package for performing ab-initio quantum-mechanical molecular dynamics (MD) simulations using pseudopotentials or the projector-augmented wave method and a plane wave basis set. The approach implemented in VASP is based on the (finite-temperature) local-density approximation with the free energy as variational quantity and an exact evaluation of the instantaneous electronic ground state at each MD time step.

VASP uses efficient matrix diagonalisation schemes and an efficient Pulay/Broyden charge density mixing. These techniques avoid all problems possibly occurring in the original Car-Parrinello method, which is based on the simultaneous integration of electronic and ionic equations of motion. The interaction between ions and electrons is 
described by ultra-soft Vanderbilt pseudopotentials (US-PP) or by the projectoraugmented wave (PAW) method. The US-PP and the PAW methods allow for a considerable reduction of the number of plane-waves per atom for transition metals and first row elements. Forces and the full stress tensor can be calculated with VASP and used to relax atoms into their instantaneous ground-state. 


\section{References}

[1] Schrödinger, Erwin. "An Undulatory Theory of the Mechanics of Atoms and Molecules". Phys. Rev., 28 (6), (1926) 1049-1070.

[2] W. Heitler and F. London, Zeitschrift für, Physik, , 44, (1927) 455-456.

[3] Born, M.; Oppenheimer, J. R. Ann Physik 1927, 84, (1927) 457-459.

[4] J. C. Slater "A Simplification of the Hartree-Fock Method" Phys. Rev., 81, (1951) $385-390$.

[5] Yong-Ki Kim. "Relativistic Self-Consistent-Field Theory for Closed-Shell Atoms". Phys. Rev. 154, (1967) 17-39.

[6] Møller, Christian; Plesset, Milton S. "Note on an Approximation Treatment for Many-Electron Systems". Phys. Rev. 46 (7), (1934) 618-622.

[7] Head-Gordon, Martin; Pople, John A.; Frisch, Michael J. "MP2 energy evaluation by direct methods". Chemical Physics Letters. 153 (6), (1988) 503-506.

[8] Jeziorski, B.; Monkhorst, H. "Coupled-cluster method for multideterminantal reference states". Physical Review A. 24 (4), (1981) 1668.

[9] Lindgren, D.; Mukherjee, Debashis. "On the connectivity criteria in the open-shell coupled-cluster theory for general model spaces". Physics Reports. 151 (2),(1987) 93-95. 
[10] Thomas, L. H. "The calculation of atomic fields". Proc. Cambridge Phil. Soc. 23 (5), (1927) 542-548.

[11] Fermi, Enrico. "Un Metodo Statistico per la Determinazione di alcune Prioprietà dell'Atomo". Rend. Accad. Naz. Lincei 6, (1927) 602-607.

[12] Hohenberg, Pierre; Walter Kohn. "Inhomogeneous electron gas". Physical Review 136 (3B), (1964) B864-B871.

[13] Kohn, W.; Sham, L. J. "Self-Consistent Equations Including Exchange and Correlation Effects". Physical Review, 140 (4A), (1965) A1133.

[14] S. H. Vosko, L. Wilk and M. Nusair. "Accurate spin-dependent electron liquid correlation energies for local spin density calculations: a critical analysis". Can. J. Phys. 58 (8), (1980) 1200-1204.

[15] J. P. Perdew and A. Zunger. "Self-interaction correction to density-functional approximations for many-electron systems". Phys. Rev. B 23 (10), (1981) 5048-5079.

[16] J. P. Perdew and Y. Wang, Phys. Rev. B 45, (1991) 13244-13248.

[17] J. P. Perdew, J. A. Chevary, S. Vosko, K. A. Jackson, M. R. Pederson, D. J. Singh, and C. Fiolhais Phys. Rev. B 46, (1992) 6671-6674.

[18] A. D. Becke. "Density-functional exchange-energy approximation with correct asymptotic behavior". Phys. Rev. A 38 (6), (1988) 3098-3100. 
[19] John P. Perdew, Kieron Burke, and Yue Wang, "Generalized gradient approximation for the exchange-correlation hole of a many-electron system" Phys. Rev. B 54, 16 (1996) 543-454.

[20] K. Kim and K. D. Jordan. "Comparison of Density Functional and MP2 Calculations on the Water Monomer and Dimer". J. Phys. Chem. 98 (40), (1994) 1008910094.

[21] P.J. Stephens, F. J. Devlin, C. F. Chabalowski and M. J. Frisch. "Ab Initio Calculation of Vibrational Absorption and Circular Dichroism Spectra Using Density Functional Force Fields". J. Phys. Chem. 98 (45), (1994) 11623-11627.

[22] Chengteh Lee, Weitao Yang and Robert G. Parr. "Development of the ColleSalvetti correlation-energy formula into a functional of the electron density". Phys. Rev. B 37 (2), (1988) 785-789.

[23] Bachelet, G. B.; Hamann, D. R.; Schlüter, M. "Pseudopotentials that work: From H to Pu", Physical Review B, 26 (8), (1982) 4199-4228.

[24] Vanderbilt, David, "Soft self-consistent pseudopotentials in generalized eigenvalue formalism", Physical Review B, 41 (11), (1990) 7892-7895.

[25] Blöchl, P.E. "Projector augmented-wave method". Physical Review B, 50 (24), (1994) 17953-17978. 
[26] W. J. Hehre, R. F. Stewart, and J. A. Pople, "Self-Consistent Molecular Orbital Methods. 1. Use of Gaussian expansions of Slater-type atomic orbitals,"J. Chem. Phys., $51,(1969)$ 2657-2664.

[27] J. B. Collins, P. v. R. Schleyer, J. S. Binkley, and J. A. Pople, "Self-Consistent Molecular Orbital Methods. 17. Geometries and binding energies of second-row molecules. A comparison of three basis sets," J. Chem. Phys., 64, (1976) 5142-51.

[28] Gill, Peter M.W. (1994). "Molecular integrals Over Gaussian Basis Functions". Advances in Quantum Chemistry, 25, (1994) 141-205.

[29] Schlegel, H.; Frisch, M. "Transformation between Cartesian and pure spherical harmonic Gaussians". International Journal of Quantum Chemistry 54 (2), (1990) 83-87.

[30] G. Kresse and J. Furthmüller. "Efficient iterative schemes for ab initio totalenergy calculations using a plane-wave basis set" Phys. Rev. B 54, (1996) 11169-11186.

[31] G. Kresse and J. Furthmüller. "Efficiency of ab-initio total energy calculations for metals and semiconductors using a plane-wave basis set" Computational Materials Science, 6(1), (1996), 15-50.

[32] Ashcroft, Neil W.; Mermin, N. David (1976). Solid state phys. New York, Holt, Rinehart and Winston. 1976, p. 135.

[33] Leighton, Robert B. "The Vibrational Spectrum and Specific Heat of a FaceCentered Cubic Crystal". Reviews of Modern Physics 20, (1) (1948) 165-174. 
Kresse, G. PhD thesis, Technische Universität Wien, 1993.

[34] G. Kresse and J. Hafner. "Ab initio molecular dynamics for liquid metals". Phys. Rev. B 47, (1993), 558-561.

[35] G. Kresse and D. Joubert. "From ultrasoft pseudopotentials to the projector augmented-wave method" Phys. Rev. B 59, (1999), 1758-1775.

[36] G. Kresse and J. Furthmüller. "Efficiency of ab-initio total energy calculations for metals and semiconductors using a plane-wave basis set" Computational Materials Science, 6 (1), (1996) 15-50.

[37] G. Kresse and J. Furthmüller. "Efficient iterative schemes for ab initio totalenergy calculations using a plane-wave basis set" Phys. Rev. B 54, (1996), 11169-11186. 


\section{Chapter 3}

\section{Interaction of Semiconducting Quantum Dots with}

\section{Dopamine and DNA nucleobases}

\subsection{Introduction}

CdSe/CdS quantum dots (QDs, usually spherical nanoparticles in the size range of 1$10 \mathrm{~nm}$ diameters ${ }^{[1]}$ ) are ideal quantum-confined nanocrystals widely used as fluorescent probes for biomedical applications owing to their unique optical and electronic properties. The intriguing feature of these semiconducting quantum dots is that the particle size determines the wavelength of fluorescence emission. One can vary fluorescence emission from the ultraviolet to the visible or near-infrared spectrum by controlling the size and the chemical composition of the QDs ${ }^{[2-6]}$. This feature has actively been utilized by scientists and engineers to design useful nanoscale devices which can label and image biological macromolecules.

In contrast to organic dyes, the $\mathrm{CdSe} / \mathrm{CdS}$ QDs can be used in the high-sensitivity multiplexed devices due to their broad excitation profiles and narrow/symmetric emission 
spectra. The CdSe/CdS QDs are also very suitable for combinatorial optical encoding, in which multiple colors and intensities are combined to encode thousands of genes, proteins or small-molecule compounds ${ }^{[7-9]}$. Nowadays, the need for biological labeling using CdSe/CdS QDs has been increasing very fast. They have been successfully used in the bioanalytical field, such as DNA hybridization detection ${ }^{[10]}$.

If an organic molecule binds to the QDs, the emission spectra will change. Different molecules may induce different changes in the emission spectra. This is the working principle of QDs detection. The sensitivity of detection (the intensity of emission spectra required to produce enough signal over noise for detection), however, is determined by the binding energy that the target organic molecule has with the imaging QDs. It is valuable knowledge for future biological imaging, if one can understand and predict what type of organic compound may induce the high intensity of emission spectra with $\mathrm{CdSe} / \mathrm{CdS}$ quantum dots (QDs). For this purpose, quantum mechanical calculations have been performed to obtain the potential energy surface describing the interaction of CdSe/CdS small QDs with the dopamine molecule and DNA nucleobases. In this study, such small clusters of $\mathrm{Cd}_{4} \mathrm{Se}_{4} / \mathrm{Cd}_{4} \mathrm{~S}_{4}$ have been used as simulation models for the CdSe/CdSe nanoparticles. Through this work, the following questions are addressed: which chemical groups have the larger binding energy with $\mathrm{Cd}_{4} \mathrm{Se}_{4} / \mathrm{Cd}_{4} \mathrm{~S}_{4}$ QDs and therefore can generate the higher intensity of emission. It is found that the CdSe QDs are indeed good for detecting the DNA molecules. This study has also provided some general guidelines for the selective detection of chemical and biological molecules. 


\subsection{Computational method}

All calculations reported here were performed within the framework of the density functional theory (DFT). We used the Vienna ab initio Simulation Package (VASP) to calculate the interaction of molecules with the $\mathrm{CdSe} / \mathrm{CdS}$ cluster. The projector augmented-wave (PAW) potentials ${ }^{[1-13]}$ and plane wave basis sets were used. Here we applied the generalized gradient approximation (GGA) and employed the exchange and correlation functional forms proposed by Perdew and Zunger ${ }^{[14,15]}$.

The computational parameters were taken from our previous studies on dopaminemetal clusters ${ }^{[16]}$. A cubic supercell is chosen which is big enough to ensure that the interaction with its image cells is negligible. The cutoff energy for the plane wave basis was set to $400 \mathrm{eV}$ and reciprocal space integrations were performed at the $\Gamma$ point. The electronic structure calculations were considered to be converged when the total energy is converged to $10^{-5} \mathrm{eV}$. The convergence criterion of force on each atom was set to 0.03 $\mathrm{eV} / \AA ̊$ for structure relaxations.

\subsection{Results and discussion}

\subsubsection{DNA nucleobase molecules}

The optimized equilibrium structures for the DNA nucleobases are shown in Figure 3.1 . 


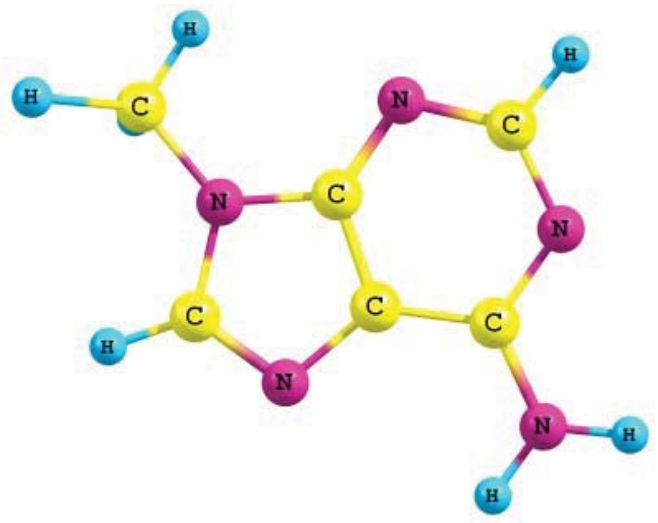

Adenine

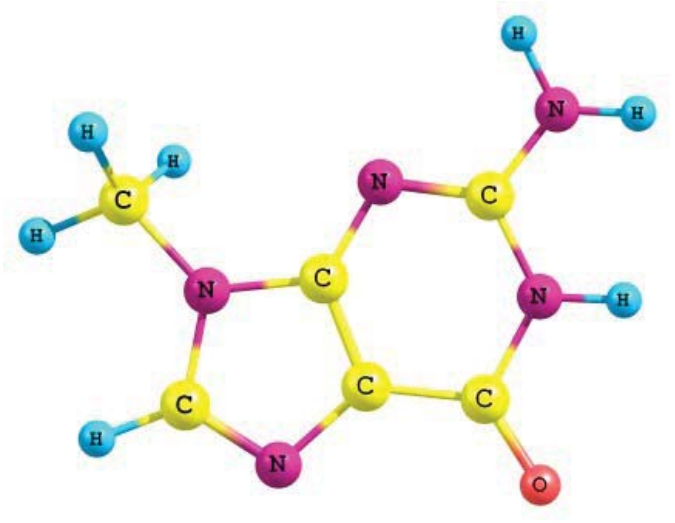

Guanine

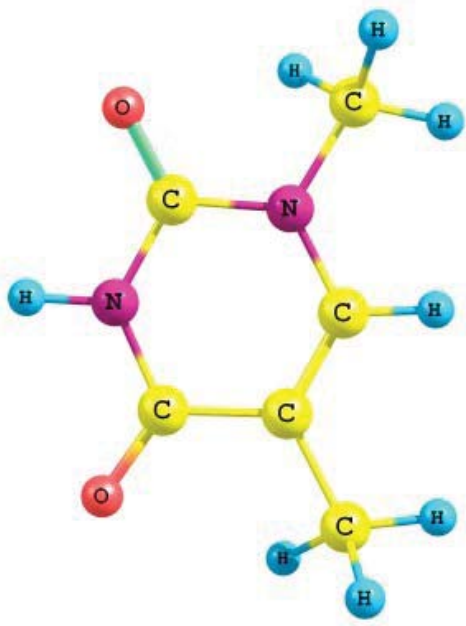

Thymine

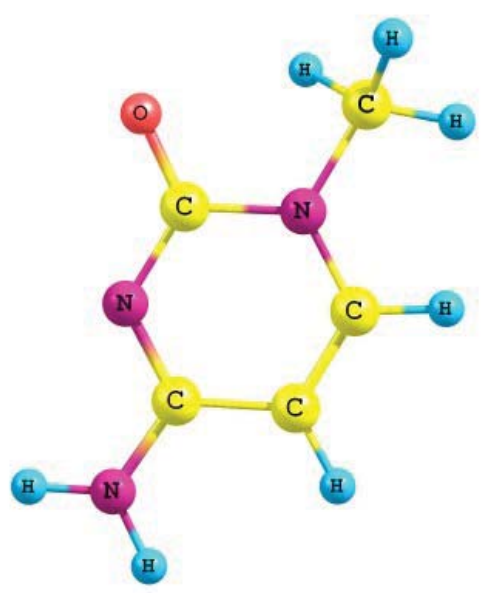

Cytosine

Figure 3.1: A ball and stick model for the DNA nucleobase (O: red, C: yellow, N: navy blue, H: blue). 


\subsection{2 $\mathrm{Cd}_{4} \mathrm{Se}_{4}$ and $\mathrm{Cd}_{4} \mathrm{~S}_{4}$ clusters}

The optimized equilibrium structures for the $\mathrm{Cd}_{4} \mathrm{Se}_{4}$ and $\mathrm{Cd}_{4} \mathrm{~S}_{4}$ clusters are shown in Figure 3.2. The lowest-energy structure found in this study has a near-cubic structure. The equilibrium structural parameters of clusters are listed in Table 3.1.

Table 3.1: The optimized geometrical parameters of Cd4Se4/ Cd4S4 small QDs.

\begin{tabular}{|c|c|c|c|c|}
\hline & & $R(\AA)$ & $\varphi_{1}(\mathrm{X}-\mathrm{Cd}-\mathrm{X})\left({ }^{\circ}\right)$ & $\varphi_{2}(\mathrm{Cd}-\mathrm{X}-\mathrm{Cd})\left(^{\circ}\right)$ \\
\hline \multirow{2}{*}{$\begin{array}{c}\mathrm{Cd}_{4} \mathrm{~S} \\
\mathrm{e}_{4}\end{array}$} & our result & 2.70 & 104.4 & 73.8 \\
\cline { 2 - 5 } & \begin{tabular}{c} 
Reference $^{\mathrm{I}}$ \\
\multirow{2}{*}{$\begin{array}{c}\mathrm{Cd}_{4} \mathrm{~S} \\
4\end{array}$}
\end{tabular} & 2.69 & 104.0 & 74.0 \\
\cline { 2 - 5 } & our result & 2.62 & 102.6 & 95.5 \\
\hline Reference & 2.61 & -- & 103.4 \\
\hline
\end{tabular}

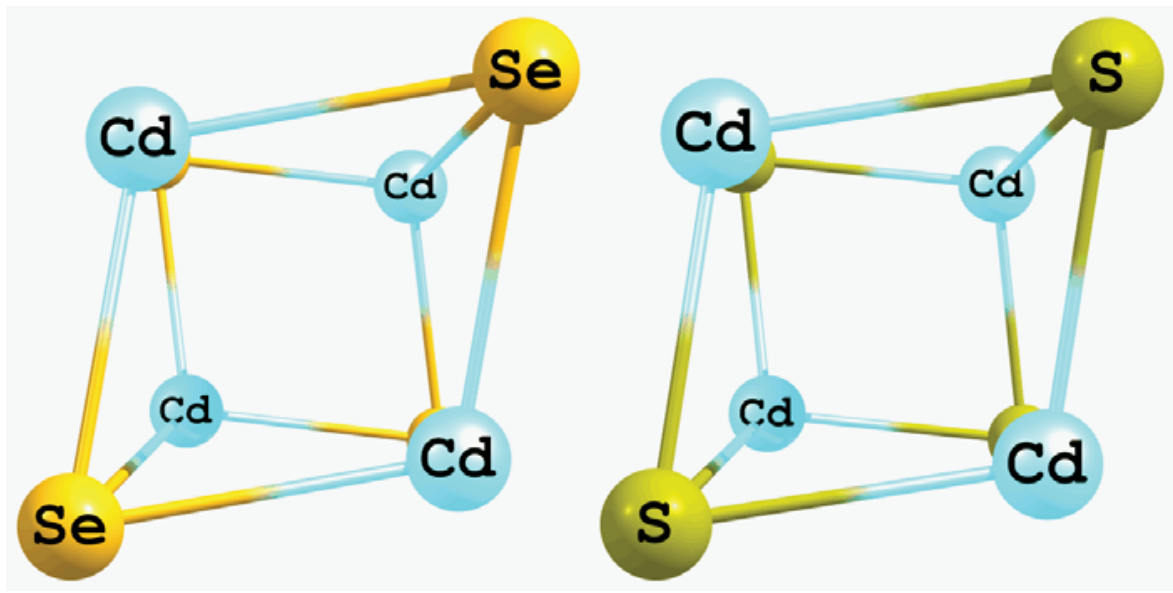

Figure 3.2: A ball and stick model for the $\mathrm{Cd}_{4} \mathrm{Se}_{4}$ and $\mathrm{Cd}_{4} \mathrm{~S}_{4}$ clusters. 


\subsubsection{Interaction of Cd4Se4 and Cd4S4 QDs with dopamine}

The dopamine molecule has three active chemical groups. That is phenyl, amido and hydroxyl. Therefore, in the study of interaction, the cluster is considered to approach the molecule towards the hydroxyl (i.e. the O-site), amido (i.e. the $\mathrm{N}$-site) and the phenyl (i.e. the Top-site) of the molecule. The directions that the QDs approach the dopamine are illustratively shown in Figure 3.3:

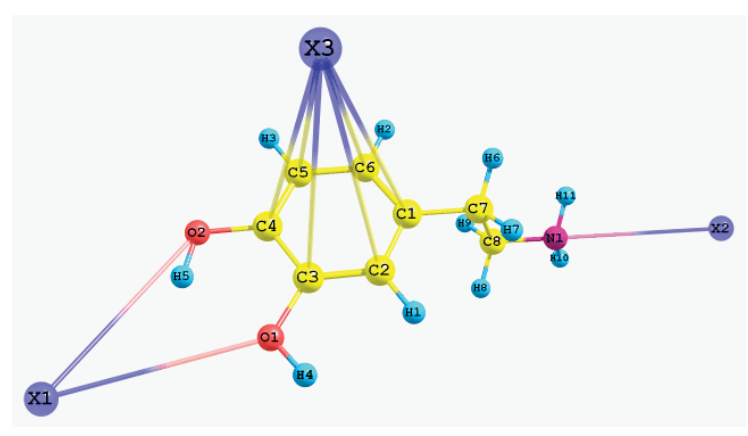

Figure 3.3 Schematic illustration of the direction of the cluster to approach the dopamine molecule (O: red, C: yellow, N: navy blue, H: blue, X: position of QDs)

In Figure 3.3, X1 shows how the QDs approach the dopamine from the Oxygen side; X2 shows how the QDs approach the dopamine from the Nitrogen side and X3 shows how the QDs approach the dopamine from the top side. When calculating the total energy of the system at a certain distance, only the correlative atoms are fixed; the other atoms are relaxed as the QDs approach the molecule. For example, if one calculates the interaction when the QDs approaches from the top site and the distance is $3 \AA$ away from the center of the phenyl ring, the six carbon atoms are fixed in position which lie in the hexagon ring as well as the semiconducting cluster. The distance between the closest 
Cadmium atom and the center of the hexagon ring is set to $3 \AA$. The reference of "zero" in energy is taken to be the energy of the system when the QDs and the molecule are far apart from each other. All the energies are taken to be the differences between the values of the calculated energies and the reference energy. The calculated potential energy surface along the three interaction paths for $\mathrm{Cd}_{4} \mathrm{Se}_{4}$ and $\mathrm{Cd} 4 \mathrm{~S} 4$ are shown in Figures 3.4 and 3.5, respectively.

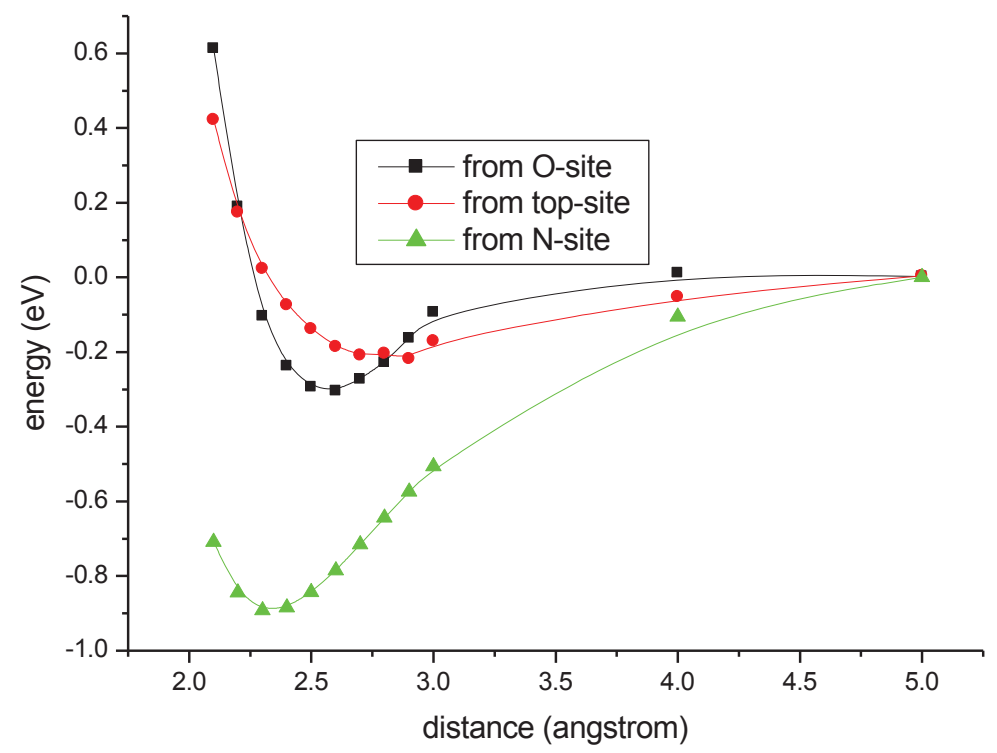

Figure 3.4: The energy surfaces describing interaction of the $\mathrm{Cd}_{4} \mathrm{Se}_{4}$ cluster with a dopamine molecule from different approaching directions towards the $\mathrm{O}$ site, the $\mathrm{N}$ site and the top site. 


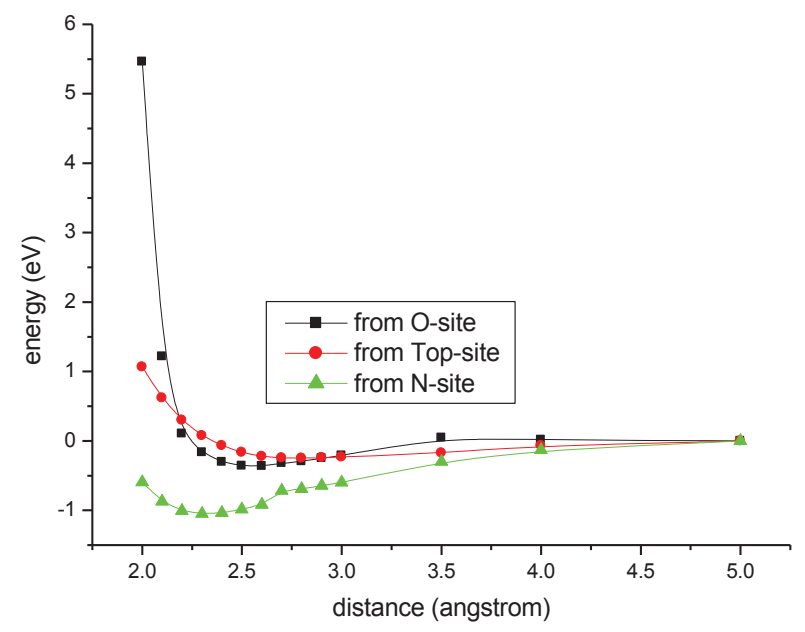

Figure 3.5: The energy surfaces describing interaction of the $\mathrm{Cd}_{4} \mathrm{~S}_{4}$ cluster with a dopamine molecule from different approaching directions towards the $\mathrm{O}$ site, the $\mathrm{N}$ site and the top site.

Table 3.2: Binding energy $\left(E_{B}\right)$ and equilibrium distance $\left(R_{B}\right)$ of the QDs-molecule complex.

\begin{tabular}{|c|r|c|c|c|}
\hline \multirow{2}{*}{ QDs } & \multicolumn{3}{|c|}{ Molecular sites } \\
\cline { 3 - 5 } & N-site & O-site & Top-site \\
\hline \multirow{2}{*}{$\begin{array}{c}\mathbf{C d}_{4} \\
\mathbf{S e}_{4}\end{array}$} & $\begin{array}{r}E_{B}, \\
\mathrm{eV}\end{array}$ & 0.89 & 0.30 & 0.21 \\
\cline { 2 - 5 } & $\begin{array}{c}R_{B,} \\
\AA\end{array}$ & 2.3 & 2.6 & 2.7 \\
\hline \multirow{2}{*}{$\mathbf{C d}_{4}$} & $\begin{array}{c}E_{B}, \\
\mathrm{eV}\end{array}$ & 1.05 & 0.36 & 0.25 \\
\cline { 2 - 5 } $\mathbf{S}_{4}$ & $\begin{array}{r}R_{B,} \\
\AA\end{array}$ & 2.3 & 2.7 & 2.8 \\
\hline
\end{tabular}

The calculated values of the binding energy $\left(E_{B}\right)$ and the equilibrium distance $\left(R_{B}\right)$ associated with the ground state configuration of the complex consisting of the QDs and molecule are given in Table 3.2. 
The energy surfaces do not show the presence of any energy barrier for all the cases considered (Figures 3.4 and 3.5), thus indicating the likelihood of exothermic reaction between the semiconductor of clusters and the dopamine molecule. For the $\mathrm{Cd}_{4} \mathrm{Se}_{4}$ and $\mathrm{Cd}_{4} \mathrm{~S}_{4}$ QDs, the calculated results show the preference for the $\mathrm{N}$-site with the binding energy of 0.89 and $1.05 \mathrm{eV}$, respectively. For the metallic clusters $\mathrm{Ag}_{13}$ and $\mathrm{Al}_{13}$, our previous studies also showed the preference for the $\mathrm{N}$-site with the binding energy of 0.67 and $0.91 \mathrm{eV}^{[22]}$. From this perspective, the semiconducting clusters of $\mathrm{Cd}_{4} \mathrm{Se}_{4}$ and $\mathrm{Cd}_{4} \mathrm{~S}_{4}$ behave very similarly to the metallic clusters of $\mathrm{Ag}_{13}$ and $\mathrm{Al}_{13}$.

The $\mathrm{Cd}$ atom has an electronic configuration of $4 d^{10} 5 s^{2}$, and is characterized by an $s$ type frontier orbital with two paired electrons in the $s$-type frontier orbital. The Cd-s orbital undergoes a small hybridization with the phenyl- $\pi$ orbital, yielding a weak interactive strength with the molecule from the top site. Likewise, the electronic configuration of the $\mathrm{Ag}$ atom is $4 d^{10} 5 s^{1}$. The $\mathrm{Ag}_{13}$ cluster is also characterized by an $s$ type frontier orbital, with one unpaired electron in the odd-numbered cluster. It has the weak interaction with the dopamine from the top site, just like CdSe/CdS QDs. The Cd atom is bonded to three Se or S atoms in the QDs. Thus, it makes part of the $5 s$ electrons of the $\mathrm{Cd}$ atom transfer to the $\mathrm{Se}$ or $\mathrm{S}$ atoms. This leads to a stronger interaction of the $\mathrm{CdSe} / \mathrm{CdS}$ cluster with the dopamine than the $\mathrm{Ag}_{13}$ cluster.

The oxygen atom generally tends to form covalent bonds with the basic metal atoms due to its high electronegativity, while the nitrogen atom tends to form coordination bonds with the metal atoms. This makes the $\mathrm{Cd}$ metal atoms have stronger interaction 
with the molecule from the $\mathrm{N}$ site than the $\mathrm{O}$ site.

On the other hand, if the $\mathrm{Cd}_{4} \mathrm{Se}_{4}$ and $\mathrm{Cd}_{4} \mathrm{~S}_{4}$ QDs interact with dopamine from the Se or $\mathrm{S}$ anions, the binding energy turns out to be close to zero for all the cases. This is due to the fact that the Se and $\mathrm{S}$ atoms are nonmetallic having high electronegativity to withdraw electrons. The outer p-orbital is almost fully occupied by electrons in the $\mathrm{CdSe} / \mathrm{CdS} \mathrm{QDs}$, so that the Se or S atom can hardly attract electrons. Therefore the binding energy is zero for such cases. This result is confirmed by the repulsive potential energy surface in Figure 3.5.
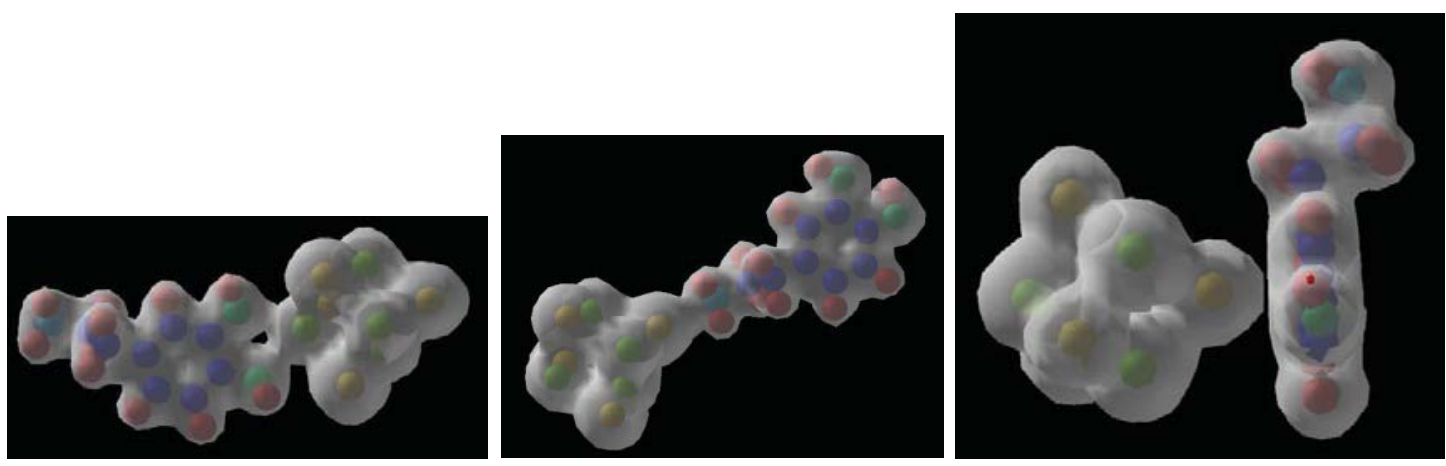

Figure 3.6: The charge density of the QDs-molecule complex showing the interaction from different sites: (a) O-site (b) N-site (c) Top-site. The contour density for (a) and (b) is $0.30 \mathrm{e} / \AA^{3}$.

\subsubsection{Interaction of $\mathrm{Cd}_{4} \mathrm{Se}_{4}$ and $\mathrm{Cd}_{4} \mathrm{~S}_{4}$ QDs with DNA Nucleobases}

The same method has been used to calculate the interaction of $\mathrm{Cd}_{4} \mathrm{Se}_{4}$ and $\mathrm{Cd}_{4} \mathrm{~S}_{4}$ QDs with DNA nucleobases. The DNA nucleobase structures and the possible directions that the cluster approaches the base molecule are shown in Figures 3.7-a to 3.10-a. In considering the static effect of the sugar-phosphate backbone of the DNA strand, we have 
only included the open sites from the six-member rings and the hydrogen bonding edges.

The calculated potential energy surfaces for $\mathrm{Cd}_{4} \mathrm{Se}_{4}$ and $\mathrm{Cd}_{4} \mathrm{~S}_{4}$ are shown in Figures 3.7-b

to $3.10-b$ :
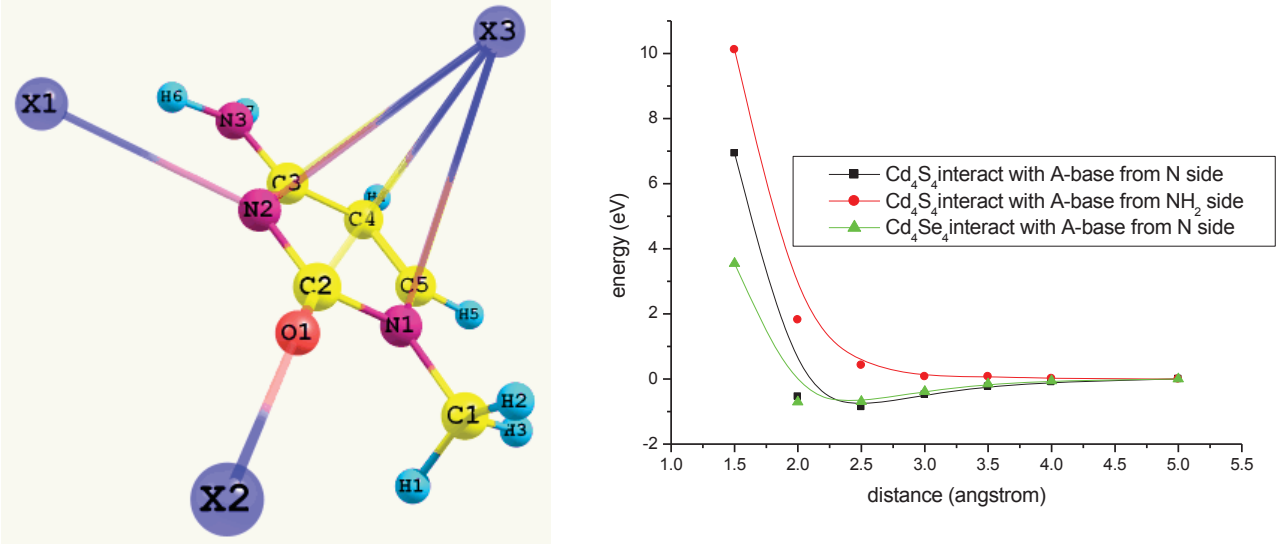

Figure 3.7: QD approaching Adenine and the energy
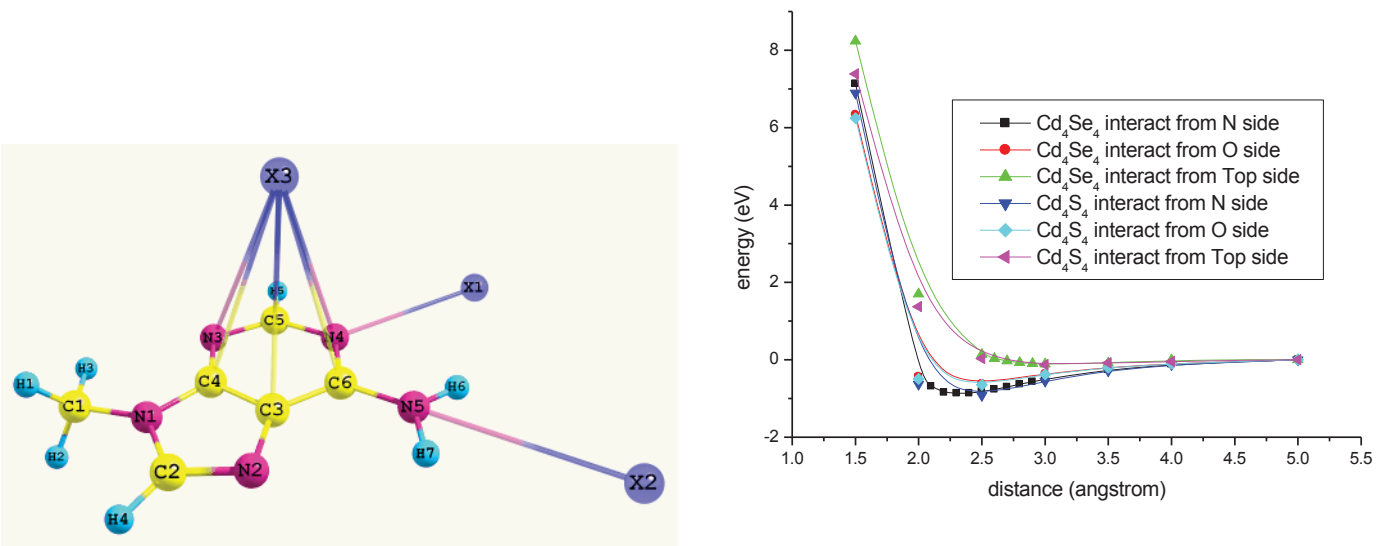

Figure 3.8: QD approaching Cytosine and the energy surface 

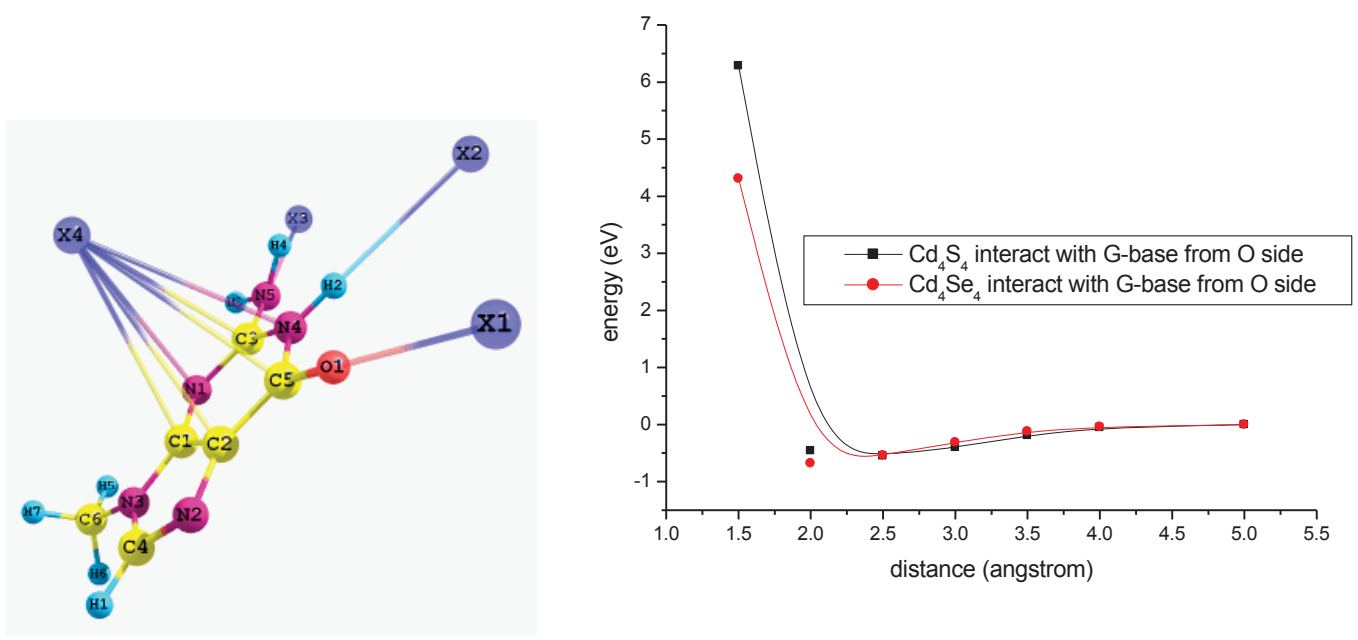

Figure 3.9: QD approaching Guanine and energy surface..
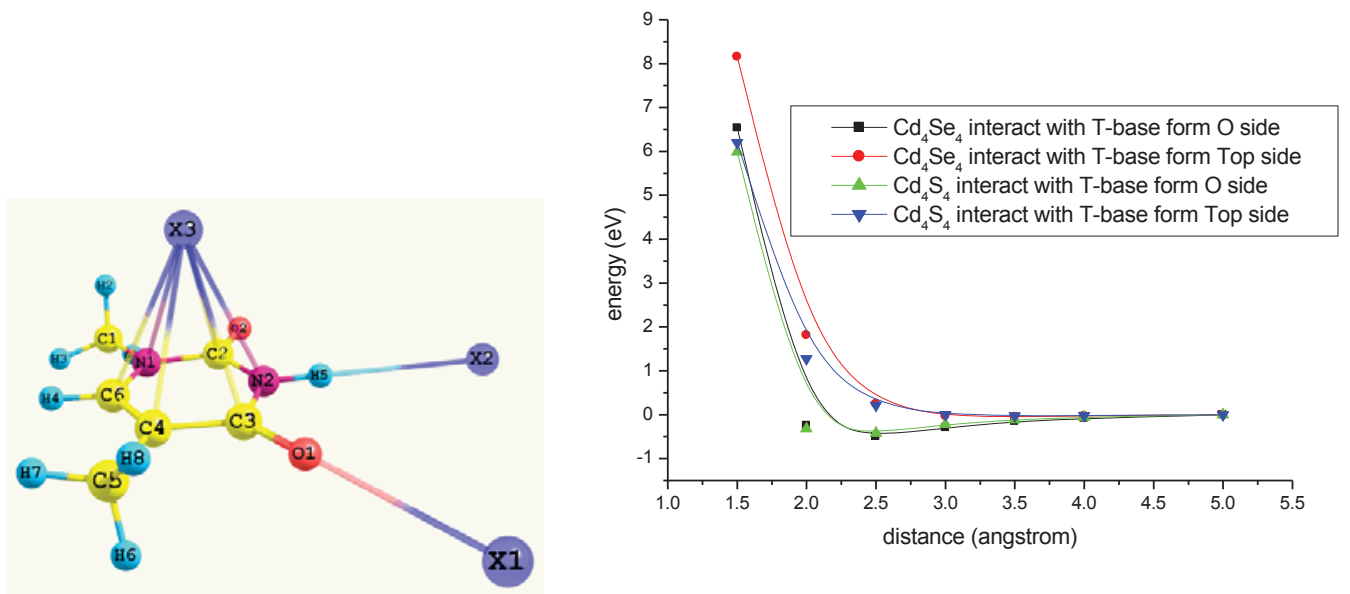

Figure 3.10: QD approaching Thymine and The energy surfaces

The calculated values of the binding energy $\left(E_{B}\right)$ and the equilibrium distance $\left(R_{B}\right)$ associated with the ground state configuration of the complex consisting of the QDs and DNA nucleobases are given in Table 3.3. 
Table 3.3: Binding energy $\left(E_{B}\right)$ and equilibrium distance $\left(R_{B}\right)$ of the cluster-DNA-base complex.

\begin{tabular}{|c|c|c|c|c|c|}
\hline \multirow{2}{*}{$\begin{array}{l}\text { Molecu } \\
\text { le }\end{array}$} & \multirow{2}{*}{ Action side } & \multicolumn{2}{|c|}{$\mathrm{CdSe}$} & \multicolumn{2}{|c|}{$\mathrm{CdS}$} \\
\hline & & $E_{B}(\mathrm{eV})$ & $R_{B} \quad(\AA)$ & $E_{B} \quad(\mathrm{eV})$ & $R_{B}(\AA)$ \\
\hline \multirow{3}{*}{ C-base } & Top & - & - & - & - \\
\hline & $\mathrm{N}$ (lie in ring) & 0.86 & 2.5 & 0.91 & 2.5 \\
\hline & $\begin{array}{l}=\mathrm{O}(\text { connect to } \\
\text { ring })\end{array}$ & 0.61 & 2.5 & 0.64 & 2.5 \\
\hline \multirow{3}{*}{ T-base } & Top & - & - & - & - \\
\hline & $=\mathrm{NH}$ & - & - & - & - \\
\hline & $\begin{array}{l}=\mathrm{O}(\text { connect to } \\
\text { ring })\end{array}$ & 0.71 & 2.5 & 0.86 & 2.5 \\
\hline \multirow{3}{*}{ A-base } & Top & - & - & - & - \\
\hline & $\mathrm{N}$ (lie in ring) & - & - & - & - \\
\hline & $\mathrm{NH}_{2}$ & - & - & - & - \\
\hline \multirow{3}{*}{ G-base } & Top & 0.55 & 2.0 & 0.68 & 2.0 \\
\hline & $=\mathrm{NH}$ (lie in ring) & & & & \\
\hline & $\begin{array}{l}=\mathrm{O}(\text { connect to } \\
\text { ring })\end{array}$ & & & & \\
\hline
\end{tabular}

From these results it is found that $\mathrm{Cd}_{4} \mathrm{Se}_{4}$ and $\mathrm{Cd}_{4} \mathrm{~S}_{4}$ QDs have strong binding interaction with the oxygen and nitrogen chemical groups in the DNA base molecules, except that the oxygen and nitrogen chemical groups having the hydrogen atoms as termini in the DNA base, where the binding energy is zero. This is because the oxygen and nitrogen atoms have paired electrons in the $p$-type orbitals, while the $\mathrm{Cd}$ atom has the empty $p$-orbitals. These p-orbitals undergo hybridization, yielding interactive strength between the $\mathrm{Cd}$ atom and the molecule. Although the oxygen atom generally has stronger 
hybridization than the nitrogen atom, the nitrogen in this case lies in the six-member ring and is part of the $\pi$-type chemical bond. This leads to the higher electron density in the nitrogen lying in the ring than in the isolated nitrogen atom. In addition to that, since the oxygen in these base molecules connects to the six-member ring, the $\pi$-conjugated chemical bonding in the ring withdraws electrons from the oxygen and makes the oxygen having lower electron density than an isolated oxygen atom. Therefore the $\mathrm{Cd}_{4} \mathrm{Se}_{4}$ and $\mathrm{Cd}_{4} \mathrm{~S}_{4}$ QDs have larger binding interaction with the nitrogen chemical group than the oxygen chemical group in these molecules.

Nonetheless, if the oxygen or nitrogen having the hydrogen termini in the DNA nucleobase, the $\mathrm{Cd}$ atom interact with the hydrogen atom at first. Since the electron of the hydrogen atom is attracted by the oxygen or nitrogen, it turns out to be electron deficient. So the hydrogen cannot donate any electron to the cadmium atom. Therefore, there is hardly any interaction between the $\mathrm{Cd}$ atom and the DNA nucleobase, and the binding energy of the CdSe/CdS QDs interacting with the DNA nucleobase from the $\mathrm{OH}$ or $\mathrm{NH}$ side is apparently zero.

\subsection{Summary}

The calculated interactive strengths for different chemical groups follow the following order: 


$$
\begin{aligned}
& \text { Phenyl }\left(\mathrm{C}_{6} \mathrm{H}_{5^{-}}\right)=\operatorname{amido}(=\mathrm{NH})(\mathrm{N} \text { lie in the ring })<\text { hydroxyl }(\mathrm{OH}) \\
& (0 \mathrm{eV}) \\
& <\text { Carbonyl }(=\mathrm{O})<\text { Nitrogen }\left(\mathrm{N} \text { lie in the phenyl ring) }<\text { amide }\left(-\mathrm{NH}_{2}\right)\right. \\
& (0.42-0.68 \mathrm{eV}) \\
& (0.71-0.86 \mathrm{eV})
\end{aligned}
$$

We can see that the $\mathrm{Cd}_{4} \mathrm{Se}_{4} / \mathrm{Cd}_{4} \mathrm{~S}_{4}$ QDs binds strongly with the DNA nucleobase molecules. That is to say, that the DNA nucleobase can coat stably on the $\mathrm{CdSe} / \mathrm{CdS}$ QDs. So a relatively strong intensity of emission spectra may be expected at a relatively low concentration of DNA nucleobase molecules in the solution. From this perspective, the $\mathrm{CdSe} / \mathrm{CdS}$ small QDs is good biological labeling material. On the other hand, if one wants to choose the target molecule to be detected, the molecule which carries the chemical group having strong binding interaction with $\mathrm{CdSe} / \mathrm{CdS}$ QDs, such as carbonyl $(=\mathrm{O})$, heterocyclic and amide $\left(-\mathrm{NH}_{2}\right)$, will have a larger sensitivity. These principles may also help in the modification of the surface of the CdSe/CdS QDs to get stronger binding with the target molecules. A lot of effort has been made in this direction ${ }^{[23-28]}$. 


\section{References}

[1] Alivisatos, A. Paul. The Journal of Physical Chemistry 100.31 (1996), 1322613239.

[2] L.H. Qu, X.G. Peng, J. Am. Chem. Soc. 124, (2002) 2049.

[3] X.H. Zhong, Y.Y. Feng, W. Knoll, M.Y. Han, J. Am.Chem.Soc. 125, (2003) 13559.

[4] R.E. Bailey, S.M. Nie, J. Am. Chem. Soc. 125, (2003) 7100.

[5] S.Kim, B.Fisher, H.J.Eisler, M.Bawendi, J. Am.Chem.Soc. 125, (2003) 11466.

[6] B.L. Wehrenberg, C.J. Wang, P. Guyot-Sionnest, J. Phys.Chem.B 106, (2002)10634.

[7] Han MY, Gao XH, Su JZ, Nie SM: Quantum-dot-tagged microbeads for multiplexed optical coding of biomolecules. Nano. Biotechnol., 19, (2001) 631.

[8] Gao XH, Nie SM: Doping mesoporous materials with multicolor quantum dots. $J$ Phys Chem B, 107, (2003) 11575.

[9] Gao XH, Nie SM: Quantum dot-encoded mesoporous beads with high brightness and uniformity: rapid readout using flow cytometry. Anal Chem, 76, (2004) 2406.

[10] W.J. Parak, D. Gerion, D. Zanchet, A.S. Woerz, T.Pellegrino, C. Micheel, S.C. Williams, M. Seitz, R.E.Bruehl, Z. Bryant, C. Bustamante, C.R. Bertozzi, A.P.Alivisatos, 
Chem.Mater. 14, (2002) 2113.

[11] G. Kresse and J. Furthmüller, "Efficient iterative schemes for ab initio totalenergy calculations using a plane-wave basis set", 54, (1996) 11169-11186.

[12] P. E. Blöchl, "Projector augmented-wave method", Phys. Rev. B, 50, (1994) 17953-979.

[13]G. Kresse and D. Joubert, "From ultrasoft pseudopotentials to the projector augmented-wave method", Phys. Rev. B, 59, (1999) 1758-1775.

[14] J. P. Perdew and A. Zunger, "Self-interaction correction to density-functional approximations for many-electron systems", Phys. Rev. B, 23, (1981) 5048-5079.

[15] J. P. Perdew, J. A. Chevary, S. H. Vosko, K. A. Jackson, M. R. Pederson, D. J. Singh, and C. Fiolhais, "Atoms, molecules, solids, and surfaces: Applications of the generalized gradient approximation for exchange and correlation", Phys. Rev. B, 46, (1992) 6671-6687.

[16] M. D. Deshpande, D. G. Kanhere, R. Pandey, "Structures, Energetics and Magnetic Properties of NinB Clusters with n=1-8,12" Phys. Rev. A, 71, (2005) 63202.

[17] Alagona, Giuliano, and Caterina Ghio. "The effect of intramolecular H-bonds on the aqueous solution continuum description of the N-protonated form of dopamine." Chemical physics 204.2 (1996), 239-249. 
[18] J. J. Urban, C. J. Cramer, G. R. Famini, J. Am. Chem. Soc. 114, (1992) 8226.

[19] Nagy, Peter I., Giuliano Alagona, and Caterina Ghio. "Theoretical studies on the conformation of protonated dopamine in the gas phase and in aqueous solution." Journal of the American Chemical Society 121.20 (1999): 4804-4815.

[20] Sabyasachi Sen and Swapan Chakrabarti "Frequency-dependent nonlinear optical properties of CdSe clusters", Phys. Rev. B 74, (2006) 205435.

[21] Chu Heying, Liu Zhaoxia, and Li Yuncai, "First-principles study of structures and electronic properties of cadmium sulfide clusters", Chinese Physics B. 17, (2008) 24782483.

[22] Liu Chunhui, He haiying \& Ravindra Pandey. "Interaction of Metallic Nanoparticles with Biological Active Molecule Dopamine", J. Phys. Chem. B, 112, (2008) 15256-15259.

[23] Chan, Warren CW, and Shuming Nie. "Quantum dot bioconjugates for ultrasensitive nonisotopic detection." Science 281.5385 (1998) 2016-2018.

[24] Willard, D. M., Carillo, L. L., Jung, J. \& Van Orden, A. CdSe-ZnS quantum dots as esonance energy transfer donors in a model protein-protein binding assay. Nano Lett., $1,(2001) 469-474$.

[25] Uyeda, H. Tetsuo, et al. "Synthesis of compact multidentate ligands to prepare stable hydrophilic quantum dot fluorophores." Journal of the American Chemical Society 
$127.11(2005) 3870-3878$.

[26] Mattheakis, Larry C., et al. "Optical coding of mammalian cells using semiconductor quantum dots." Analytical biochemistry 327.2 (2004) 200-208.

[27] Ballou, Byron, et al. "Noninvasive imaging of quantum dots in mice." Bioconjugate chemistry 15.1 (2004) 79-86.

[28] Kim, Sungjee, and Moungi G. Bawendi. "Oligomeric ligands for luminescent and stable nanocrystal quantum dots." Journal of the American Chemical Society 125.48 (2003) 14652-14653. 



\section{Chapter 4}

\section{Structure of Membrane Lipids: DMPC and DMPE}

\subsection{Introduction}

A biological membrane separates a cell from its surrounding environment. Its function depends on the continuity and integrity of the lipid since a lipid bilayer makes up the skeleton structure of a membrane. Also, over $70 \%$ weight of membrane belongs to the lipid bilayer. ${ }^{[1]}$ Consequently, knowledge of the structure of a lipid bilayer is essential to understand the functionality of a biological membrane. ${ }^{[2]}$ In practice, details of the structural configuration of a lipid bilayer can be obtained by the X-ray diffraction measurements. ${ }^{[3-8]}$ However, it is not easy to perform experiments which require excellent quality of single crystals of lipid bilayers together with low temperature conditions. On the other hand, access to the high-performance computational facilities has become available with the modest resources leading to a routine use of theoretical methods to study biomolecular crystals. For example, atomistic simulation methods such as classical molecular dynamics (MD) and Monte Carlo (MC) have been applied to simulate a variety of interesting phenomena ranging 
from self-assembly, ${ }^{[9-12]}$ phase transition, ${ }^{[13-15]}$ interactions of lipid with inorganic materials, ${ }^{[16-21]}$ and biological molecules. ${ }^{[22-28]}$ In general, accuracy and reliability of atomistic methods rely on the quality of semi-empirical force fields adopted in the simulations.

It is well recognized that first-principles quantum mechanical methods can provide a detailed description of a crystal without relying on the availability of experimental results or semi-empirical force fields. Recently, such an effort was reported for a biological system where a first-principles study of the membrane lipid dipalmitoyl phosphatidylcholine (DPPC) molecule and monolayer was performed to investigate their molecular and electronic structural properties. The local density approximation to density functional theory was used to examine the structural features of the water interfacing the lipid. ${ }^{[29]}$

In this chapter, the structures of two types of phospholipid crystals, namely 1,2Dimyristoyl-sn-glycero-3-phosphorylcholine (DMPC) and 1,2-Dimyristoyl-snglycero-3-phosphorylethanolamine (DMPE) are studied using first-principles quantum mechanical methods. DMPC is chosen as a model system to benchmark the results of our calculations since it is well-characterized experimentally. DMPC is composed of the hydrophilic choline linked to the hydrophobic alkyl chains, whereas the hydrophilic aniline is linked to the hydrophobic alkyl chains for DMPE. Recognizing that the biological functionality not only relies on the molecular crystal structure, but also external environmental factors, the role of hydration effects in predicting the crystal 
structures of DMPC and DMPE membrane lipids is also examined. Specifically, we are intrigued by the fact that the germs whose membrane is mainly made up of PE type lipid tend to die in a dry surroundings. Therefore, the crystal structures of DMPE under dry and aqueous conditions are both calculated to examine its functionality under different environmental conditions. Furthermore, we seek to determine the degree of reactivity of DMPC and DMPE via calculations of the dipole moments knowing that the phospholipids line up their hydrophilic and hydrophobic groups and build the so-called bilayer configurations. Furthermore the electric double layer configuration of a membrane lipid is accompanied by a large dipole moment due to the positive and negative charge separation in its structure.

A lipid bilayer configuration is composed of two monolayers where their hydrophilic and hydrophobic groups point outward and inward, respectively. Recognizing that the constituent monolayers are identical and weakly interacting, we begin with a simulation model of a lipid consisting of a single layer which allows us to perform first principles calculations on the membrane lipids with modest computational resources. Note that the interactions of a lipid bilayer with the environment primarily occur at the hydrophilic group sites. Since the choice of a monolayer configuration retains the reactive sites, the simulation model can still determine the degree of the reactivity of the membrane lipids.

\subsection{Simulation model}

The generalized gradient approximation (GGA) to density functional theory (DFT) has 
been employed as implemented in the VASP program. ${ }^{[30]}$ The projector augmented-wave $(\mathrm{PAW})^{[31-33]}$ potentials and plane wave basis sets together with the exchange and correlation functional forms proposed by Perdew and Zunger ${ }^{[34,35]}$ are used for calculations. This functional form has been well tested in the simulation of a wide variety of crystalline materials. ${ }^{[36]}$ It has also been pointed out that the choice of the functional form including van der Waals functional does not modify significantly the geometrical configuration for DPPC. ${ }^{\text {[29] }}$

The energy cutoff of the plane wave was taken to be $400 \mathrm{eV}$. During the optimization procedure, both size of the supercell and internal coordinates of all the atoms were relaxed. The criteria of $10^{-5} \mathrm{eV}$ for energy convergence and $0.01 \mathrm{eV} / \AA$ for the convergence of forces on each atom in the unit cell were chosen. For the hydrated DMPC and DMPE, a water molecule is added in the supercell which is in the vicinity of the hydrophilic group. Based on calculations with a higher plane wave cut off and the tighter convergence criteria, we find the parameters used are likely to introduce an error of the order of $0.1 \mathrm{eV}$ in the total energy of the system.

\subsection{Results and discussion}

\subsubsection{DMPC and DMPE}

Figure 4.1 shows the ball and stick models for the primary molecular structures of DMPC and DMPE. The hydrophobic alkyl chains are linked to the hydrophilic choline and aniline chains for DMPC and DMPE, respectively. In order to simulate the hydrated 
crystal, packing of the alkyl chains with one water molecule in an orthorhombic array is considered. ${ }^{[37]}$

In general, the head group of a lipid consists of two equally oppositely charged ionic chemical groups, e.g. the choline and phosphate groups of DMPC carry positive and negative charges, respectively. In each layer of the membrane lipid, molecules are arranged side by side to form the so-called electric double layer where all of the positive groups lie on a plane or shell and the entire negative groups lie on the other plane or shell. The functionality of a membrane lipid then depends on the details of the structure of its electric double layer. 


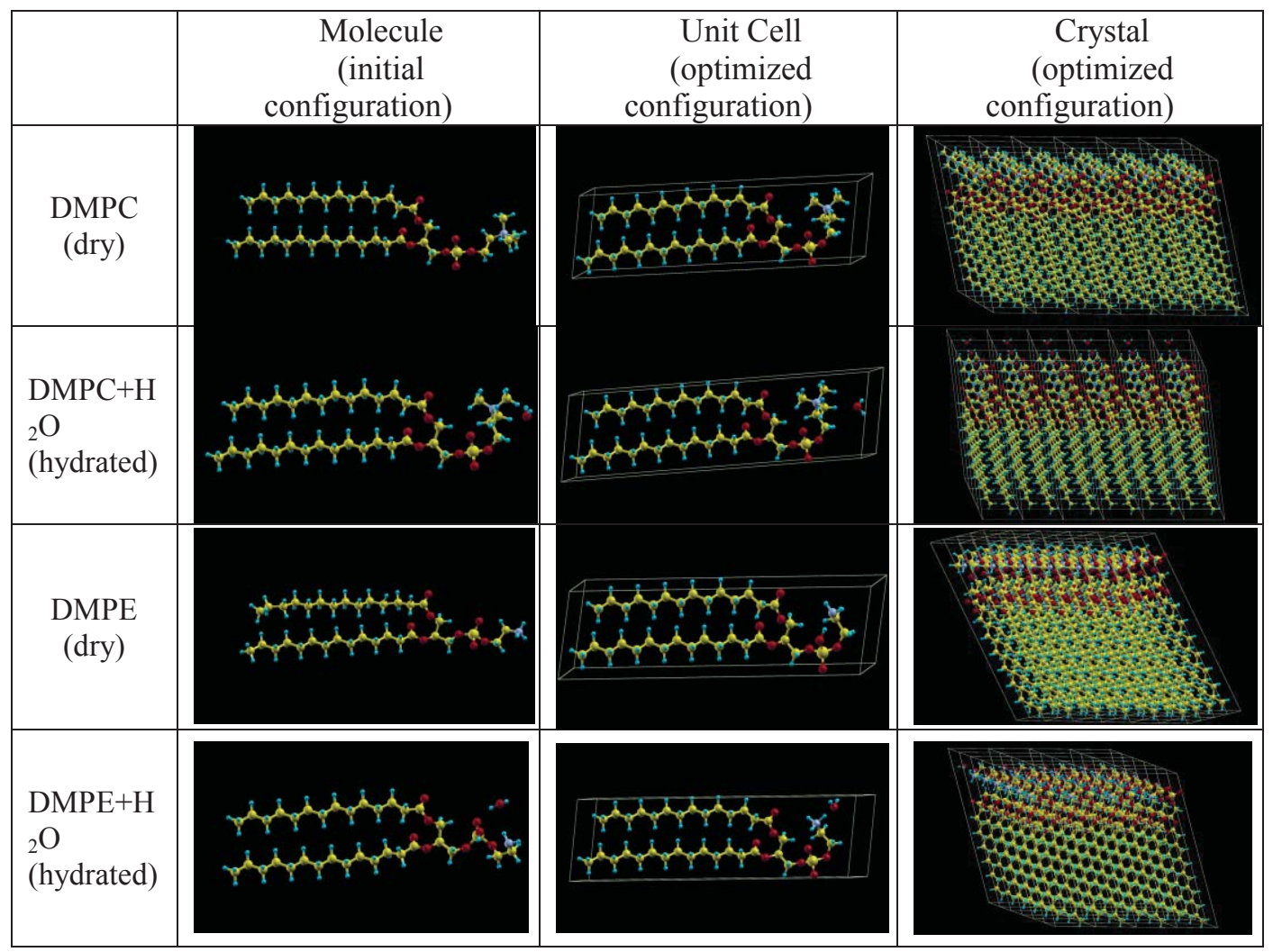

Figure 4.1: The molecular configurations and crystal structures of DMPC and DMPE. (Atomic symbols: C-(small) yellow, O-red, P-(large) yellow, N-(large) dark blue and H-(small) light blue.)

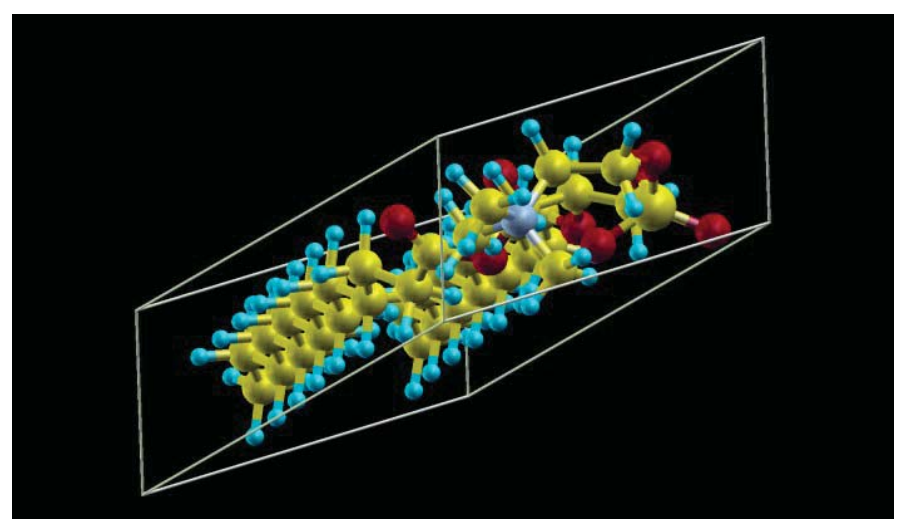

Figure 4.2: The triclinic unit cell of DMPC. Atomic symbols: C-(small) yellow, O-red, P-(large) yellow, $\mathrm{N}$-(large) dark blue and $\mathrm{H}$-(small) light blue. 
Table 4.1: The calculated lattice parameters of the DMPC and DMPE crystals.

\begin{tabular}{|c|c|c|c|c|c|c|c|}
\hline & $\begin{array}{c}a \\
(\AA)\end{array}$ & $\begin{array}{c}b \\
(\AA)\end{array}$ & $\begin{array}{c}\mathrm{c} \\
(\AA)\end{array}$ & $\begin{array}{c}\alpha \\
\left({ }^{\circ}\right)\end{array}$ & $\begin{array}{c}\gamma \\
\left({ }^{\circ}\right)\end{array}$ & $\begin{array}{c}\text { Area per } \\
\text { lipid (eq. 1) } \\
\left(\AA^{2}\right)\end{array}$ & $\begin{array}{c}\text { Volu } \\
\text { me } \\
\left(\AA^{3}\right)\end{array}$ \\
\hline DMPC (dry) & 4.4 & 8.4 & 28.6 & 95.2 & 65.5 & 36.5 & 3719 \\
\hline $\begin{array}{c}\text { DMPC: } \mathrm{H}_{2} \mathrm{O} \\
\text { (hydrated) }\end{array}$ & 4.4 & 8.5 & 29.8 & 95.2 & 65.1 & 37.2 & 3967 \\
\hline DMPE (dry) & 4.3 & 8.4 & 27.9 & 97.6 & 66.1 & 36.1 & 3617 \\
\hline $\begin{array}{c}\text { DMPE: } \mathrm{H}_{2} \mathrm{O} \\
\text { (hydrated) }\end{array}$ & 4.3 & 8.4 & 27.7 & 97.9 & 66.1 & 36.1 & 3586 \\
\hline
\end{tabular}

The area per lipid (A) on a plane projected perpendicular to the alkyl chains can be defined as follows:

$$
A=a \times b \cdot \sin \alpha
$$

Where $a$ and $b$ represent the magnitude of the unit cell vectors in $x$ and $y$ directions, respectively, and $\alpha$ is the angle between $a$ and $b$ (Figure 4.2). We note that the average area per lipid is one of most fundamental characteristics of the membrane lipids. ${ }^{[37]}$

The equilibrium lattice parameters of the DMPC and DMPE monolayer crystals obtained at the GGA-DFT level of theory are given in Table 4.1. Note that no geometrical constraints were imposed during the optimization process to obtain the crystal structures. A noticeable effect of the hydration is seen only for DMPE. A comparison between the calculated structural parameters of the dry and hydrated crystals shows a slight expansion of the molecular area $(\approx 2 \%)$ and volume $(\approx 5 \%)$ for the hydrated DMPE. However, this is not the case for the hydrated DMPC where addition of water does not lead to significant changes in the structural parameters of the unhydrated crystal. The predicted differences 
in the hydration behavior of DMPC and DMPE can be understood in terms of the nature of their polar headgroups and will be discussed in the next section.

A direct comparison of the calculated values with the corresponding experimental values of the lattice parameters cannot be made as calculations are performed at $0 \mathrm{~K}$ whereas the experiments are generally performed at about $300 \mathrm{~K} \cdot{ }^{[38-40]}$ In spite of this, the calculated value $36.5 \AA^{2}$ compares reasonably well with the experimental value of 39 $\AA^{2}$ obtained at $283 \mathrm{~K}$. A note of caution is that a lipid bilayer exists in three different phases: the fluid phase in the biological environment, the gel phase and the crystalline phase. The area per lipid generally varies in a wide range for these different phases. Both temperature and humidity can also affect the degree of hydration of these phases. Thus, there exists a great challenge in getting a consensus of the calculated values with the experimentally measured values for the membrane lipids. In addition to it, the relative uncertainties in the experimental determination of the area per lipid tend to be large. We note that the simulation study based on the molecular dynamics method reported the area per lipid to be 50-60 $\AA^{2}$ for both DMPC and DMPE lipid bilayers in the fluid phases, ${ }^{[37]}$ which is significantly larger than the calculated values in the more compact and solidified crystalline phase. 


\subsubsection{Polar headgroups of DMPC and DMPE}
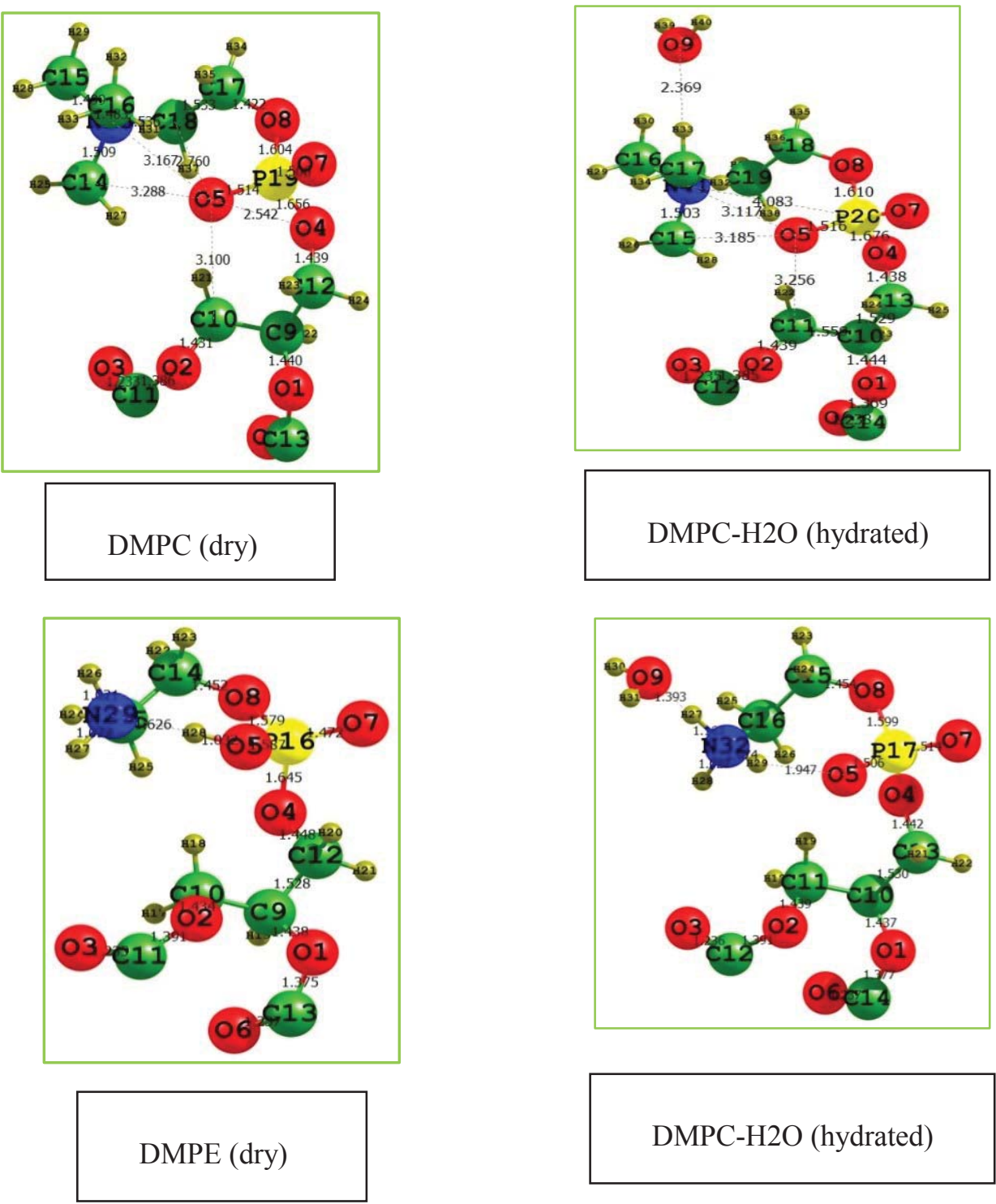

DMPC-H2O (hydrated)

DMPC-H2O (hydrated)

Figure 4.3: The hydrophilic heads of DMPC and DMPE. Atomic symbols: C-green, O-red, Pyellow, N-dark blue and H-(small) dark yellow. 
Table 4.2: The calculated bond lengths of the hydrophilic heads of DMPC and DMPE as shown in

Figure 4.3.

\begin{tabular}{|c|c|c|c|c|c|}
\hline Bond & $\begin{array}{c}\text { DMPC } \\
(\text { dry }) \\
(\AA)\end{array}$ & $\begin{array}{c}\text { DMPC } \\
(\text { hydrated }) \\
(\AA)\end{array}$ & Bond & $\begin{array}{c}\text { DMPE } \\
(\text { dry }) \\
(\AA)\end{array}$ & $\begin{array}{c}\text { DMPE } \\
\text { (hydrated }) \\
(\AA)\end{array}$ \\
\hline $\mathrm{H}_{2} \mathrm{O}-\mathrm{H} 33$ & - & 2.37 & $\mathrm{H}_{2} \mathrm{O}-\mathrm{H} 27$ & - & 1.39 \\
\hline $\mathrm{N}-\mathrm{C} 16$ & 1.50 & 1.50 & $\mathrm{~N}-\mathrm{H} 27$ & 1.02 & 1.16 \\
\hline $\mathrm{N}-\mathrm{C} 17$ & 1.48 & 1.49 & $\mathrm{~N}-\mathrm{H} 28$ & 1.02 & 1.03 \\
\hline $\mathrm{N}-\mathrm{C} 15$ & 1.51 & 1.50 & $\mathrm{~N}-\mathrm{H} 29$ & 1.63 & 1.07 \\
\hline O5-C15 & 3.29 & 3.19 & O5-H29 & 1.03 & 1.95 \\
\hline $\mathrm{P}-\mathrm{O} 5$ & 1.51 & 1.52 & $\mathrm{P}-\mathrm{O} 5$ & 1.59 & 1.51 \\
\hline $\mathrm{P}-\mathrm{O} 4$ & 1.66 & 1.68 & $\mathrm{P}-\mathrm{O} 4$ & 1.65 & 1.66 \\
\hline $\mathrm{P}-\mathrm{O} 7$ & 1.51 & 1.50 & $\mathrm{P}-\mathrm{O} 7$ & 1.47 & 1.51 \\
\hline $\mathrm{P}-08$ & 1.60 & 1.61 & $\mathrm{P}-\mathrm{O} 8$ & 1.58 & 1.60 \\
\hline
\end{tabular}

The calculated structural parameters, including bond lengths and angles for the hydrophilic heads of DMPC and DMPE are given in Table 4.2. The choline nitrogen site of DMPC exhibits nearly symmetric 3-fold coordination of the nitrogen atom with the methyl groups having the bond distance of about $1.5 \AA$ (Figure 4.3). This is also the case with the aniline group of DMPE where the nitrogen atom has two neighboring $\mathrm{H}$ atoms with the bond distances of about $1.02 \AA$ (Figure 4.3). 


\subsubsection{Hydration effects: DMPC and DMPE}

Addition of a water molecule near the polar headgroup leads to distinctly different configuration for DMPE, though the hydrated DMPC retains the structural features of the unhydrated DMPC. The absence of the water molecule in the case of DMPE facilitates a conversion of a zwitterion to a pair of neutral groups, where the ammonium group $\left(-\mathrm{NH}_{3}{ }^{+}\right)$loses a proton turning into the amino group $\left(-\mathrm{NH}_{2}\right)$ and the phosphate radical group $\left[-\mathrm{O}-\mathrm{P}(\mathrm{O})_{2}-\mathrm{O}\right]^{-}$gets a proton forming the neutral $[-\mathrm{O}-\mathrm{P}(\mathrm{O})(\mathrm{OH}) \mathrm{O}-]$. A direct evidence of this transition is found for DMPE where $R_{\mathrm{H}(28)-\mathrm{O}(5)}$ decreases from 1.626 $\AA$ to $1.033 \AA$. Thus, the optimized configuration of DMPE does not retain the electric double layer structure in the dry condition. It is worth noting that the amine group lies in the plane owing to loss of the electric double layer structure, and has negligible steric hindrance for the amino group $\left(\mathrm{NH}_{2}\right)$. Nonetheless, in the case of DMPC, calculations

find the distance of the water molecule to be $2.37 \AA$ suggesting the existence of a relatively weak interaction between water and lipid. This is further confirmed by negligible changes in the structural parameters of DMPC after the hydration. Thus, DMPC retains nearly the same electric double layer structure in both dry and hydrated conditions.

\subsubsection{Dipole moment}

A bilayer structure leads to the results that the lipid has a strong dipole moment along the direction perpendicular to the plane of the membrane lipid, since all of the positive 
groups lie on a plane and the entire negative groups lie on the other plane of the membrane lipid. Since the adsorption capacity of a membrane depends mainly on the intermolecular forces, and thereby on the dipole moment, the magnitude of the dipole moments then essentially determines the degree of reactivity of the membrane lipid.

The calculated values of the dipole moments of DMPC and DMPE are shown in Table 4.3. The center of the cell is chosen as the reference point for these calculations. For the unhydrated DMPC monolayer crystals, our calculated value of the dipole energy is -0.66 $\mathrm{kcal} / \mathrm{mol}$. Thus, the calculated value of $-1.32 \mathrm{kcal} / \mathrm{mole}$ for the bilayer crystal compares well with the value of $-1.21 \mathrm{kcal} / \mathrm{mol}$ obtained using the linearized Poisson-Boltzmann equation. ${ }^{[41]}$ The electric dipole moment of DMPC remains nearly the same in dry and hydrated conditions, though the same is not true for DMPE. There is a nearly 3 times increase in the calculated dipole moment for DMPE under the hydrated conditions. Note that the dipole moment of a water molecule is about $\sim 2 \mathrm{D}$. 
Table 4.3: The calculated components of dipole moments and the dipole energy per monolayer of DMPC and DMPE.

\begin{tabular}{|c|c|c|c|c|c|}
\hline \multirow{2}{*}{ Dipole Moment } & \multicolumn{3}{|c|}{ Components } & \multirow{2}{*}{$\begin{array}{l}\text { Total } \\
\text { (D) }\end{array}$} & \multirow{2}{*}{$\begin{array}{r}\text { Energy } / \\
\text { monolayer } \\
(\mathrm{kcal} / \mathrm{mol})\end{array}$} \\
\hline & $\alpha(D)$ & $\beta(D)$ & $\gamma(\mathrm{D})$ & & \\
\hline DMPC (dry) & -4.08 & 0.58 & -12.30 & 13.50 & -0.66 \\
\hline $\begin{array}{r}\text { DMPC } \\
\text { (hydrated) }\end{array}$ & -6.96 & 0.29 & -11.82 & 14.54 & -0.60 \\
\hline DMPE (dry) & -0.38 & 0.04 & -4.95 & 5.02 & -0.10 \\
\hline $\begin{array}{r}\text { DMPE } \\
\text { (hydrated) }\end{array}$ & -1.97 & 0.10 & -15.27 & 15.69 & -0.98 \\
\hline
\end{tabular}

A closer examination of Table 5.3 reveals the dominance of the $\gamma$-component of the dipole moment for both DMPC and DMPE. Such a large value of the dipole moment thus facilitates the membrane lipid to have strong adsorption ability, since the $\gamma$-component represents the direction perpendicular to the plane of the alkyl chain of the phospholipid system. Furthermore, a noticeable change in the $\gamma$-component is seen under hydrated conditions for DMPE reflecting the changes in the structural configuration of the polar aniline group induced by the hydrated conditions.

\subsubsection{Electron density and Bader charge}

Figures 4.4 (a)-(d) show the electron density contour plots on the plane containing the hydrophilic headgroups of DMPC and DMPE. The electron density contours on the plane containing the choline head-group do not show any noticeable changes in going from dry to hydrated conditions. However, this is not the case for DMPE. Under the dry condition, 
$\mathrm{H}$ which lies between $\mathrm{N}$ (of the $-\mathrm{NH}_{2}$ group) and $\mathrm{O}$ (of the [-OP(O)(OH)O-] group) forms a bond with $\mathrm{O}$ at the amine site of DMPE, thus leading to the loss of the electric double layer structure. Nevertheless, under the hydration condition, transfer of a proton results into $\left[-\mathrm{OP}(\mathrm{O})_{2} \mathrm{O}-\right]^{-}$and $\left[-\mathrm{NH}_{3}\right]^{+}$carrying negative and positive charges, respectively.

Table 4.4 lists the Bader charge calculated for DMPC and DMPE under the dry and hydrated conditions. A negative value of $\left(\mathrm{Q}_{\text {hydrated }}^{\prime}-\mathrm{Q}_{\text {dry }}\right)$ represents the gain of electron for a given atom under hydrated condition. 


\section{Choline site}

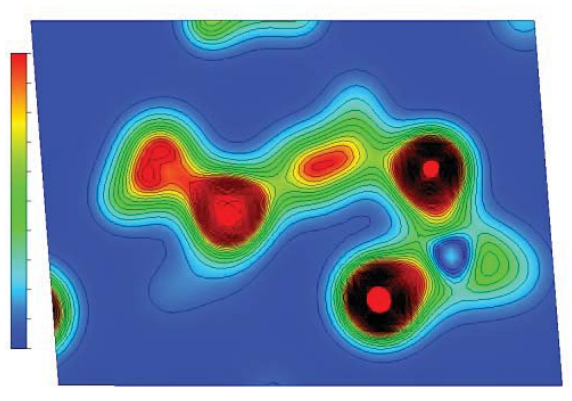

DMPC (dry)

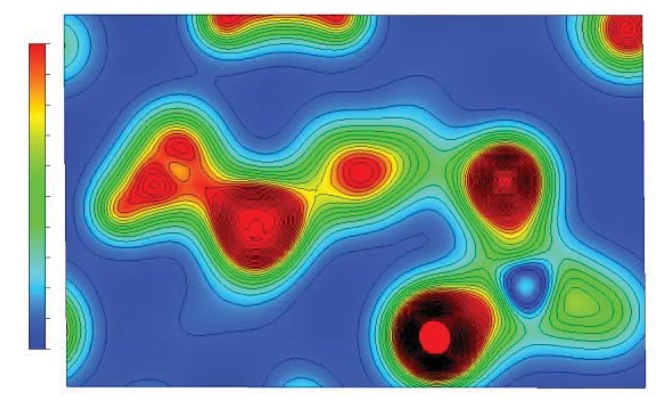

DMPC (hydrated)

\section{Amine site}

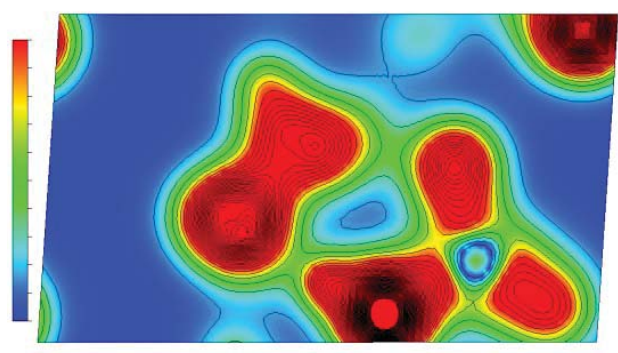

DMPE (dry)

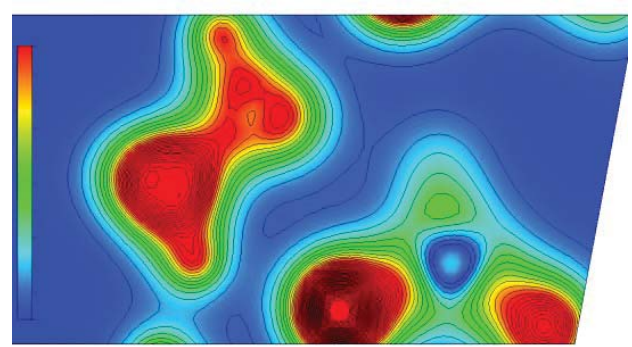

DMPE (hydrated)

Figure 4.4: The electron density contour plots on the plane containing the hydrophilic headgroups of DMPC and DMPE.

Analysis of Bader charge confirms the changes in the electron density contours of DMPE under the hydrated conditions. Thus, $\mathrm{N}$ of the amine group appears to have a higher electron density around it. Note that the $\mathrm{O}$ atom of a water molecule forms a bond with one of the hydrogens of the amine group making the corresponding $\mathrm{N}-\mathrm{H}$ bond to be relatively weak. As a result, the $\mathrm{N}$ atom attracts $\mathrm{H}$ of the phosphate group to form 
zwitterion. This is not the case with DMPC where the N atom bonds with three methyl radicals shield the hydration effect owning to the large steric hindrance and the lack of hydrogen bonds among them. Although the PC type and the PE type of phospholipids have similar backbone structures, the chemical and physical properties are distinguishably different due to the difference in the nature of their headgroups.

Table 4.4: Bader charge (e) of DMPC and DMPE calculated under dry and hydrated conditions.

\begin{tabular}{|c|c|c|c|c|c|c|}
\hline $\begin{array}{l}\text { Ato } \\
\mathrm{m}\end{array}$ & $\begin{array}{c}Q_{\text {dry }} \\
\text { (DMPC) }\end{array}$ & $\begin{array}{l}\mathrm{Q}_{\text {hydrated }}^{\prime} \\
\text { (DMPC) }\end{array}$ & $\begin{array}{l}\mathrm{Q}^{\prime}-\mathrm{Q} \\
(\mathrm{DMPC})\end{array}$ & $\begin{array}{c}Q_{\text {dry }} \\
\text { (DMPE) }\end{array}$ & $\begin{array}{l}Q_{\text {hydrated }}^{\prime} \\
\text { (DMPE } \\
\text { ) }\end{array}$ & $\begin{array}{c}\text { Q'-Q } \\
\text { (DMPE) }\end{array}$ \\
\hline $\mathrm{O}_{4}$ & -1.2685 & -1.2667 & 0.0018 & -1.2626 & -1.2619 & 0.0007 \\
\hline $\mathrm{O}_{5}$ & -1.4680 & -1.4670 & 0.0010 & -1.4196 & -1.4758 & -0.0562 \\
\hline $\mathrm{O}_{7}$ & -1.4344 & -1.4405 & 0.0061 & -1.4121 & -1.4376 & -0.0255 \\
\hline $\mathrm{O}_{8}$ & -1.2583 & -1.2544 & 0.0039 & -1.2787 & -1.2467 & 0.0320 \\
\hline $\mathrm{P}$ & 3.5313 & 3.5287 & 0.0026 & 3.5650 & 3.5429 & -0.0221 \\
\hline $\mathrm{N}$ & -0.8885 & -0.8885 & 0 & -1.1647 & -1.2121 & -0.0474 \\
\hline $\begin{array}{l}\mathrm{H}_{10} \\
6\end{array}$ & - & - & - & 0.4355 & 0.5438 & 0.1083 \\
\hline $\begin{array}{l}\mathrm{H}_{10} \\
7\end{array}$ & - & - & - & 0.4361 & 0.4561 & 0.0200 \\
\hline $\begin{array}{l}\mathrm{H}_{10} \\
8 \\
\end{array}$ & - & - & - & 0.6448 & 0.5310 & -0.1138 \\
\hline $\mathrm{C}_{16}$ & 0.0995 & 0.1138 & -0.0143 & - & - & - \\
\hline $\mathrm{C}_{17}$ & 0.1055 & 0.1509 & -0.0454 & - & - & - \\
\hline $\mathrm{C}_{20}$ & 0.1119 & 0.0879 & 0.0240 & - & - & - \\
\hline
\end{tabular}




\subsection{Summary}

In this study, we have calculated the crystalline structures of monolayers of DMPE and DMPC using first principles method. It is found that DMPC maintains an electric double-layer structure even under dry conditions, and its structure does not change much in the presence of water. However, the molecular structure of DMPE gets modified losing its electric double layer structure in dry conditions. In an aqueous environment, polarity of water facilitates a proton transfer to form the ammonia-phosphate zwitterion. Thereby, DMPE recovers its electric double layer as seen in the biological environment. Thus, a loss of the bilayer structure in dry conditions appears to explain why the organisms mainly composed of PE-type membrane lipid tend to die in the dry surroundings. Furthermore, we find the electric double layer structure to be associated with a large dipole moment which leads to a relative strong interaction for DMPE with adsorbents in the aqueous environment.

The results of our theoretical study have revealed the critical role of the aqueous environment in ensuring the electric double layer structure for some of the membrane lipids, which in turn is important in realizing the biological functionality of the membrane lipids. The degree of the reactivity of membranes appears to depend on the magnitude of the dipole moment determined primarily by the headgroups of the membrane lipids. 


\section{References}

[1] R.E. Wuthier, "Lipid-Composition of Isolated Epiphyseal Cartilage Cells, Membranes and Matrix Vesicles", Biochimica Et Biophysica Acta, 409 (1975) 128-143.

[2] A.P. Lyubartsev, A.L. Rabinovich, "Recent development in computer simulations of lipid bilayers", Soft Matter, 7 (2011) 25-39.

[3] M. Elder, P. Hitchcock, R. Mason, G.G. Shipley, "Refinement Analysis of Crystallography of Phospholipid, 1,2-Dilauroyl-Dl-Phosphatidylethanolamine, and Some Remarks on Lipid-Lipid and Lipid-Protein Interactions", Proceedings of the Royal Society of London Series a-Mathematical Physical and Engineering Sciences, 354 (1977) $157-170$.

[4] R.H. Pearson, I. Pascher, "Molecular-Structure of Lecithin Dihydrate", Nature, 281 (1979) 499-501.

[5] G. Vanderkooi, "Crystal-Structure Refinement Using Analytical Derivatives of the Energy Function - Application to 1,2-Dilauroyl-Dl-Phosphatidylethanolamine", Journal of Physical Chemistry, 94 (1990) 4366-4372.

[6] H. Hauser, I. Pascher, S. Sundell, Conformation of Phospholipids - CrystalStructure of a Lysophosphatidylcholine Analog, Journal of Molecular Biology, 137 (1980) 249-264. 
[7] Y.L. Wu, K. He, S.J. Ludtke, H.W. Huang, "X-Ray-Diffraction Study of Lipid Bilayer-Membranes Interacting with Amphiphilic Helical Peptides - Diphytanoyl Phosphatidylcholine with Alamethicin at Low Concentrations", Biophysical journal, 68 (1995) 2361-2369.

[8] M.C. Wiener, S.H. White, "Structure of a Fluid Dioleoylphosphatidylcholine Bilayer Determined by Joint Refinement of X-Ray and Neutron-Diffraction Data .3. Complete Structure", Biophysical journal, 61 (1992) 434-447.

[9] J.F. Kraft, M. Vestergaard, B. Schiott, L. Thogersen, "Modeling the Self-Assembly and Stability of DHPC Micelles Using Atomic Resolution and Coarse Grained MD Simulations", Journal of Chemical Theory and Computation, 8 (2012) 1556-1569.

[10] K.A. Scott, P.J. Bond, A. Ivetac, A.P. Chetwynd, S. Khalid, M.S.P. Sansom, "Coarse-grained MD simulations of membrane protein-bilayer self-assembly", Structure, $16(2008) 621-630$.

[11] O. Farago, N. Gronbech-Jensen, "Simulation of Self-Assembly of Cationic Lipids and DNA into Structured Complexes", Journal of the American Chemical Society, 131 (2009) 2875-2881.

[12] R.S. Saksena, P.V. Coveney, "Self-assembly of ternary cubic, hexagonal, and lamellar mesophases using the lattice-Boltzmann kinetic method", Journal of Physical Chemistry B, 112 (2008) 2950-2957. 
[13] J.J.G. Casares, L. Camacho, M.T.M. Romero, J.J.L. Cascales, "Effect of the barometric phase transition of a DMPA bilayer on the lipid/water interface. An atomistic description by molecular dynamics simulation", Journal of Physical Chemistry B, 111 (2007) 13726-13733.

[14] J.J.L. Cascales, T.F. Otero, A.J.F. Romero, L. Camacho, "Phase transition of a DPPC bilayer induced by an external surface pressure: From bilayer to monolayer behavior. A molecular dynamics simulation study, Langmuir", the ACS journal of surfaces and colloids, 22 (2006 5818-5824).

[15] S.L. Duncan, I.S. Dalal, R.G. Larson, "Molecular dynamics simulation of phase transitions in model lung surfactant monolayers", Biochimica Et Biophysica ActaBiomembranes, 1808 (2011) 2450-2465.

[16] J. Wong-Ekkabut, S. Baoukina, W. Triampo, I.M. Tang, D.P. Tieleman, L. Monticelli, "Computer simulation study of fullerene translocation through lipid membranes", Nature Nanotechnology, 3 (2008) 363-368.

[17] M.A. Villarreal, S.B. Diaz, E.A. Disalvo, G.G. Montich, "Molecular dynamics simulation study of the interaction of trehalose with lipid membranes, Langmuir", the ACS journal of surfaces and colloids, 20 (2004) 7844-7851. 
[18] D. Bedrov, G.D. Smith, H. Davande, L.W. Li, "Passive transport of C-60 fullerenes through a lipid membrane: A molecular dynamics simulation study", Journal of Physical Chemistry B, 112 (2008) 2078-2084.

[19] R.S.G. D'Rozario, C.L. Wee, E.J. Wallace, M.S.P. Sansom, "The interaction of C60 and its derivatives with a lipid bilayer via molecular dynamics simulations", Nanotechnology, 20 (2009).

[20] L.W. Li, H. Davande, D. Bedrov, G.D. Smith, "A molecular dynamics simulation study of C-60 fullerenes inside a dimyristoylphosphatidylcholine lipid bilayer", Journal of Physical Chemistry B, 111 (2007) 4067-4072.

[21] J.Z. Liu, A.J. Hopfinger, "Identification of possible sources of nanotoxicity from carbon nanotubes inserted into membrane bilayers using membrane interaction quantitative structure-activity relationship analysis", Chemical Research in Toxicology, 21 (2008) 459-466.

[22] P.C. Biggin, M.S.P. Sansom, "Interactions of alpha-helices with lipid bilayers: a review of simulation studies", Biophysical Chemistry, 76 (1999) 161-183.

[23] S.W. Chiu, S. Subramaniam, E. Jakobsson, "Simulation study of a gramicidin/lipid bilayer system in excess water and lipid. I. Structure of the molecular complex", Biophysical journal, 76 (1999) 1929-1938. 
[24] P.J. Bond, M.S.P. Sansom, "The simulation approach to bacterial outer membrane proteins (Review)", Molecular Membrane Biology, 21 (2004) 151-161.

[25] P. La Rocca, P.C. Biggin, D.P. Tieleman, M.S.P. Sansom, "Simulation studies of the interaction of antimicrobial peptides and lipid bilayers", Biochimica Et Biophysica Acta-Biomembranes, 1462 (1999) 185-200.

[26] M.R.R. de Planque, J.A. Killian, "Protein-lipid interactions studied with designed transmembrane peptides: role of hydrophobic matching and interfacial anchoring (Review)", Molecular Membrane Biology, 20 (2003) 271-284.

[27] M.B. Boggara, R. Krishnamoorti, "Partitioning of Nonsteroidal Antiinflammatory Drugs in Lipid Membranes: A Molecular Dynamics Simulation Study", Biophysical journal, 98 (2010) 586-595.

[28] M.S.P. Sansom, K.A. Scott, P.J. Bond, "Coarse-grained simulation: a highthroughput computational approach to membrane proteins", Biochemical Society Transactions, 36 (2008) 27-32.

[29] A. Mashaghi, P. Partovi-Azar, T. Jadidi, N. Nafari, P. Maass, M.R.R. Tabar, M. Bonn, H.J. Bakker, "Hydration strongly affects the molecular and electronic structure of membrane phospholipids", Journal of Chemical Physics, 136 (2012). 
[30] G. Kresse, J. Furthmuller, "Efficiency of ab-initio total energy calculations for metals and semiconductors using a plane-wave basis set", Computational Materials Science, 6 (1996) 15-50.

[31] G. Kresse, J. Furthmuller, "Efficient iterative schemes for ab initio total-energy calculations using a plane-wave basis set", Physical Review B, 54 (1996) 11169-11186.

[32] P.E. Blochl, "Projector Augmented-Wave Method", Physical Review B, 50 (1994) 17953-17979.

[33] G. Kresse, D. Joubert, "From ultrasoft pseudopotentials to the projector augmented-wave method", Physical Review B, 59 (1999) 1758-1775.

[34] J.P. Perdew, A. Zunger, "Self-Interaction Correction to Density-Functional Approximations for Many-Electron Systems", Physical Review B, 23 (1981) 5048-5079.

[35] J.P. Perdew, J.A. Chevary, S.H. Vosko, K.A. Jackson, M.R. Pederson, D.J. Singh, C. Fiolhais, "Atoms, Molecules, Solids, and Surfaces - Applications of the Generalized Gradient Approximation for Exchange and Correlation", Physical Review B, 46 (1992) 6671-6687.

[36] W.H. Zhu, X.W. Zhang, T. Wei, H.M. Xiao, "DFT studies of pressure effects on structural and vibrational properties of crystalline octahydro-1,3,5,7-tetranitro-1,3,5,7tetrazocine", Theor Chem Acc, 124 (2009) 179-186. 
[37] A.A. Gurtovenko, M. Patra, M. Karttunen, I. Vattulainen, "Cationic DMPC/DMTAP lipid bilayers: Molecular dynamics study", Biophysical journal, 86 (2004) 3461-3472.

[38] M.C. Wiener, R.M. Suter, J.F. Nagle, "Structure of the Fully Hydrated Gel Phase of Dipalmitoylphosphatidylcholine", Biophysical journal, 55 (1989) 315-325.

[39] M. Suwalsky, L. Duk, "X-Ray Studies on Phospholipid-Bilayers .7. Structure Determination of Oriented Films of L-Alpha-Dimyristoylphosphatidylethanolamine (Dmpe)", Makromolekulare Chemie-Macromolecular Chemistry and Physics, 188 (1987) 599-606.

[40] C. Zheng, G. Vanderkooi, "Molecular-Origin of the Internal Dipole Potential in Lipid Bilayers - Calculation of the Electrostatic Potential", Biophysical journal, 63 (1992) 935-941.

[41] R.R. Gabdoulline, C. Zheng, G. Vanderkooi, "Molecular origin of the internal dipole potential in lipid bilayers: Role of the electrostatic potential of water", Chemistry and Physics of Lipids, 84 (1996) 139-146. 


\section{Chapter 5}

\section{Prediction of Peptide Crystal Structures}

\subsection{Introduction}

It is well known that structure and composition of a crystal essentially determines its properties ${ }^{[1-3]}$. Therefore, an accurate determination of the crystal structure is of fundamental importance and still poses great challenges, especially for organic crystals. In general, it is determined using X-ray or neutron diffraction methods. Nonetheless, it is not always easy to obtain the three-dimensional structure of an organic crystal by experimental methods. For example, it is quite labor intensive to synthesize an ultra-pure protein crystal as a first step to the X-ray diffraction experiment. It is also known that formation of organic crystals is extremely sensitive to the delicate changes in both its chemical environment and thermal conditions. In addition, refinement of the experimentally-derived crystal structures also requires a lot of input variables based on the physics and chemistry of the given material.

Theoretical studies in recent years have emerged to predict structure of small organic crystals based on only the chemical composition or molecular formula without prior knowledge of the experimental results ${ }^{[4-11]}$. But, compared to inorganic crystals, such 
theoretical studies on organic crystals are rather scarce and far from maturity. This is due to the fact that it is usually rather difficult to predict structures of organic crystals using only the structural formula. Gavezotti clearly stated "No" in his paper entitled "Are Crystal Structure Predictable?" and pointed out that small energy differences between different hypothetical crystal structures are the limiting factor for an accurate prediction of the crystal structure using theoretical methods ${ }^{[12]}$. This dogma has been challenged many times. For instance, the Cambridge Crystallographic Data Centre (CCDC) has organized several blind tests in the past asking people to identify the unknown crystal structure of a material ${ }^{[13-18]}$. Over the years, several results point towards the success achieved in predicting the crystal structure of small organic crystals, but the unambiguous prediction of organic crystal structures using computational methods remains a great challenge for organic molecules with highly flexible chains. Moreover, these blind tests demonstrated that first-principles density functional theory (DFT) methods were prohibitively expensive in terms of the computational resources ${ }^{[12]}$. It is thus desirable to develop a scheme that has modest cost without losing much of the accuracy required for correct ranking of the molecular conformers for a given organic crystal.

A well-known family of cyclic di-amino acid peptides (CDAPs) is taken as the test case to examine the accuracy and reliability of our method. These peptides are also known as dioxopiperazines, piperazine-2, 5-diones or diketopiperazines (DKPs) derivatives which are amongst the "simplest" peptide derivatives commonly found in nature ${ }^{[19]}$. Furthermore, several conformations of these peptides also allow one to grasp 
the characteristics of this newly-developed scheme in determining its accuracy and reliability since the crystal structure of cyclo (S-Met-S-Met) is well characterized ${ }^{[20,21]}$. In addition, the crystal structure of its $\mathrm{R}$ enantiomer di-amino acid peptide cyclo (R-Met$\mathrm{R}-\mathrm{Met}$ ) is also computed to confirm that these $\mathrm{R} / \mathrm{S}$ enantiomer crystals share similar physical and chemical properties.

\subsection{Computational method}

The structural prediction of an organic crystal is distinct from that of the inorganic crystal in the fact that one needs to successfully predict the preferred conformation of molecules in the unit cell. The task is far beyond achieving the symmetry and lattice parameters of the unit cell. Conformations of the peptide cyclo crystal were optimized in the framework of the periodic supercell approach using the Vienna ab initio Simulation Package (VASP) ${ }^{[22] . ~ T h e ~ v a n ~ d e r ~ W a a l s ~(v d W) ~ i n t e r a c t i o n s ~ d e s c r i b e d ~ v i a ~ a ~ p a i r-w i s e ~}$ force field in the DFT-D2 method of Grimme were also included ${ }^{[23]}$. The projector augmented-wave potentials ${ }^{[24,25]}$ and plane wave basis sets with the energy cut off of 400 $\mathrm{eV}$ were used. During the optimization procedure, the sizes of the cell as well as the internal coordinates of all the atoms were fully relaxed. The criteria of $10^{-5} \mathrm{eV}$ for energy convergence and $0.01 \mathrm{eV} / \AA$ for the convergence of forces on each atom in the unit cell were chosen for calculations. Note that the initially chosen molecular conformations of the peptide cyclo were taken from DFT calculations ${ }^{[26]}$ performed at the PW91/6$31 \mathrm{G}(\mathrm{d}, \mathrm{p})$ level of theory as implemented in the Gaussian09 program. 


\subsection{Results and discussion}

\subsubsection{The cyclo (S-Met-S-Met) molecule}

The cyclo (S-Met-S-Met) molecule consists of two $\left(-\mathrm{CH}_{2}-\mathrm{CH}_{2}-\mathrm{S}-\mathrm{CH}_{3}\right)$ groups and one $(=\mathrm{CH}-\mathrm{CO}-\mathrm{N}-)_{2}$ ring as shown in Figure 6.1.

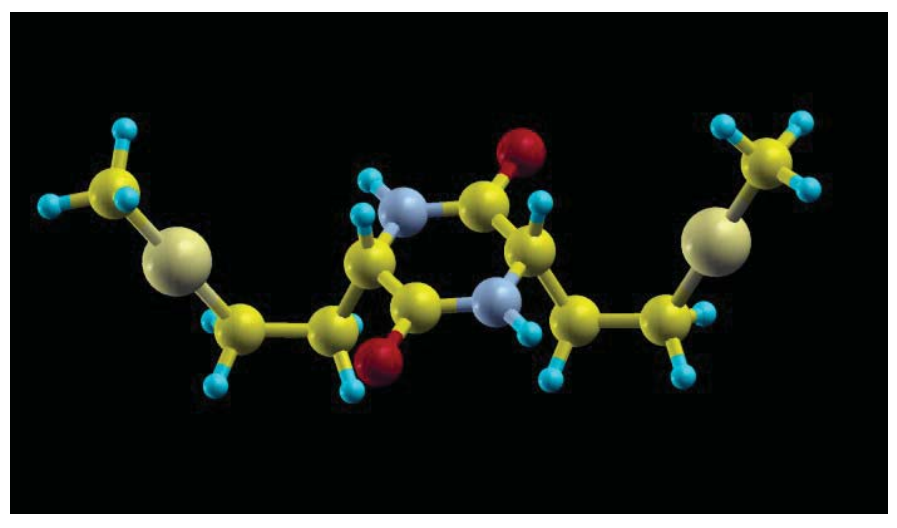

Figure 5.1: A schematic illustration of the conformations of the cyclo (S-Met-S-Met) molecule.

\subsubsection{The cyclo (S-Met-S-Met) crystal}

In order to make the theoretical prediction of a crystal structure knowing only the structural formula, we begin with a supercell representing the conventional unit cell of the crystal constructed using a graphic code, Chemcraft1.6. During the geometry optimization, internal coordinates of atoms and lattice parameters of the unit cell (i.e. $a, b$, $c, \alpha, \beta, \gamma)$ are fully relaxed. Note that the structural prediction of a crystal composed of organic/biological molecules is distinct from that of inorganic crystals in the fact that one needs to successfully predict the conformations of molecules in the unit cell. The task is 
far beyond achieving the symmetry and lattice parameters of the unit cell. In the following, it is shown that one could successfully predict a crystalline structure by a careful consideration of packing of the molecules in the crystalline form and molecular conformation change in the presence of the crystal field, which can be controlled by the dihedral angle of the ring with respect to the ab plane of the unit cell of the crystal.

A basic feature of the first-principles approach is that the temperature is assumed to be $0 \mathrm{~K}$. Consequently, one can imagine that following the minimum-energy search scheme, only local minimum-energy configuration nearest to the initial configuration can be obtained. In other words, the method cannot drive the molecules to cross the energy barrier by itself to find the global minimum of the configuration. Therefore, sampling all the probable configurations in the initial set-up is critical to the success of our method.

\subsubsection{Step I: Packing of the molecule in the crystalline form-choice of the dihedral} angle

Considering the fact that the six-member ring of the molecule determines the packing of molecules in its crystalline form, the initial choice of the inclination of this ring with respect to the $a b$ plane ( $\phi_{\text {ring ab }}$ ) of the unit cell is crucial for determining the optimum crystalline structure. $\phi_{\text {ring_ab }}$ of $0^{\circ}$ makes the ring of cyclo molecule parallel to the $a b$ plane of the unit cell, whereas $\phi_{\text {ring_ab }}$ of $45^{\circ}$ makes the ring along the diagonal direction of the unit cell in an initial cell setup where $a=b$ (Figure 5.2). Table 5.1 shows the results of our calculations on 'S-Met-S-Met' molecule for which we considered two packing 
arrangements with $\phi_{\text {ring_ab }}$ of $0^{\circ}$ and $45^{\circ}$. The results find that the packing of 'S-Met-SMet' molecule in the crystalline form is preferred when $\phi_{\text {ring_ab }}$ is set to $45^{\circ}$ initially. One unique feature of unit cell selection for a molecular crystal is that one has to include the whole molecule as one integrated piece in the cell. Given any shape of a parallelepiped, the diagonal direction provides the longest span to accommodate a longitudinal molecule, as found in this case. Since all lattice parameters are free to change, the choice of $45^{\circ}$ in an original tetragonal cell actually allows the cell to relax into any shape and any angle it prefers. 
Table 5.1: Step I: Choice of $\phi_{\text {ring_ab }}$ for cyclo (S-Met-S-Met).

\begin{tabular}{|c|c|c|c|}
\hline \multicolumn{2}{|c|}{} & $\phi_{\text {ring_ab }}=0^{\circ}$ & $\phi_{\text {ring } \_ \text {ab }}=45^{\circ}$ \\
\hline \multicolumn{2}{|c|}{ Space group } & P1 & P1 \\
\hline \multirow{4}{*}{$\begin{array}{c}\text { Lattice Parameters } \\
\left(\AA,^{\circ}\right)\end{array}$} & $\mathrm{a}$ & 4.81 & 4.79 \\
\cline { 2 - 4 } & $\mathrm{b}$ & 5.82 & 5.22 \\
\cline { 2 - 4 } & $\mathrm{c}$ & 12.91 & 13.21 \\
\cline { 2 - 4 } & $\alpha$ & 99.3 & 99.5 \\
\cline { 2 - 4 } & $\gamma$ & 93.9 & 96.8 \\
\cline { 2 - 4 } & $\gamma$ & 109.1 & 106.9 \\
\hline \multicolumn{2}{|c|}{ Unit cell volume $\left(\AA^{3}\right)$} & 329.80 & 307.18 \\
\hline \multicolumn{2}{|r|}{ Density $\left(\mathrm{g} / \mathrm{cm}^{3}\right)$} & 1.34 & 1.44 \\
\hline \multicolumn{2}{|r|}{ Total energy $(\mathrm{eV})$} & -198.58 & -199.24 \\
\hline
\end{tabular}

\begin{tabular}{|c|c|c|}
\hline & $\phi_{\text {ring_ab }}=0^{o}$ & $\phi_{\text {ring_ab }}=4^{\circ}$ \\
\hline $\begin{array}{c}\text { Ground State } \\
\text { Configuration } \\
\text { (isolated molecule) }\end{array}$ & & \\
\hline $\begin{array}{c}\text { Configuration: Initial } \\
\text { Guess }\end{array}$ & & \\
\hline $\begin{array}{c}\text { Configuration: After } \\
\text { optimization }\end{array}$ & 12 & 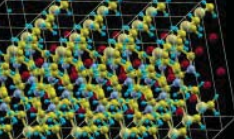 \\
\hline
\end{tabular}


Figure 5.2: Step I: Packing orientation preference of cyclo (S-Met-S-Met) where the dihedral angle of the ring with respect to the $a b$ plane of the unit cell of the crystal

$\phi_{\text {ring_ab }}$ is set to $0^{\circ}$ and $45^{\circ}$ respectively. Atomic symbols: $\mathrm{C}$ in yellow, $\mathrm{N}$ in navy blue, $\mathrm{O}$ in red, $\mathrm{S}$ in gray and $\mathrm{H}$ in light blue.

\subsubsection{Step-II: Choice of orientation of two groups in the crystalline structure}

After finding the preferred $\phi_{\text {ring_ab }}$ to be $45^{\circ}$ for packing in a crystal, we now would like to determine the orientation of two $\left(-\mathrm{CH}_{2}-\mathrm{CH}_{2}-\mathrm{S}_{-}-\mathrm{CH}_{3}\right)$ groups with respect to the center ring in the crystalline form. Thus, three crystal configurations are considered for further calculations: (i) 'cis-S-Met-cis-S-Met' (ii) 'trans-S-Met-cis-S-Met', and (iii) 'transS-Met-trans-S-Met'. Here the cis or trans configuration is referred to the configuration formed by four atoms: the $\mathrm{C}$ in the ring connecting to the chain, the first, second $\mathrm{C}$ and the $\mathrm{S}$ atom on the chain.

Table 5.2 lists the calculated structural parameters of the configurations of the 'S-MetS-Met' crystal shown in Figure 5.3. Interestingly, all configurations relax to the triclinic crystal lattice with nearly same shape of the unit cells; differences in the angles $\alpha, \beta$, and $\gamma$ are less than $3^{\circ}$ (Table 5.2). On the other hand, values of lattice constants are not similar, and a lower unit cell volume for the 'trans-cis' configuration relative to the other configurations is predicted. Likewise, the total energy of the molecular crystal in the diagonal configuration - 'trans-cis' is also found to be lower than that in the parallel configuration - "cis-cis'. Thus, the 'trans-cis' configuration is chosen abiding to the principles of minimal energy for further structure minimization. It is interesting to see 
that the most stable conformation of the isolated (S-Met-S-Met) molecule is not the one preferred in the crystalline form. This is because besides the intramolecular interaction, the intermolecular interaction between molecules plays an important role in stabilizing the crystal structure.

Table 5.2: Step II: The optimized configurations of cyclo (S-Met-S-Met) crystals having different conformations of their two $\left(-\mathrm{CH}_{2}-\mathrm{CH}_{2}-\mathrm{S}-\mathrm{CH}_{3}\right)$ groups with $\phi_{\text {ring_ab }}$ of $45^{\circ}$.

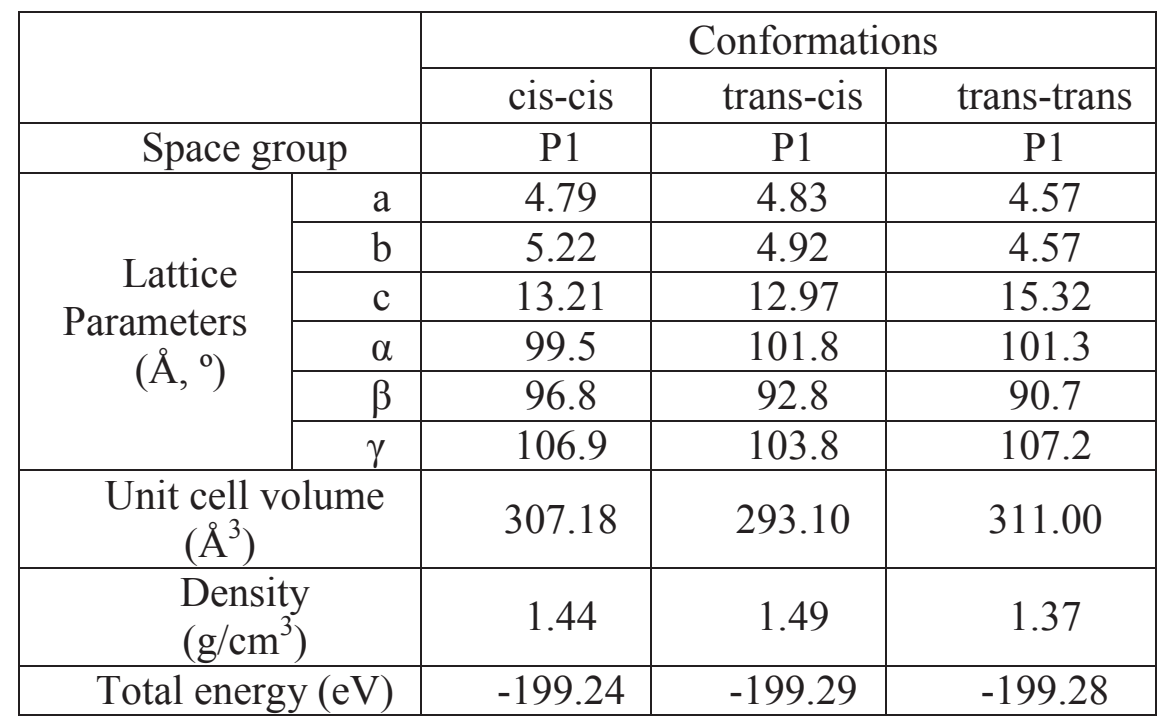




\begin{tabular}{|c|c|c|c|}
\hline & $\begin{array}{l}\text { Initial Configuration } \\
\text { of a molecule in the cell }\end{array}$ & $\begin{array}{c}\text { Initial Guess } \\
\text { Configuration } \\
\text { (crystal) }\end{array}$ & $\begin{array}{c}\text { Ground State } \\
\text { Configuration } \\
\text { (crystal) }\end{array}$ \\
\hline $\begin{array}{r}\text { 'tran } \\
\text { s-trans' }\end{array}$ & & & 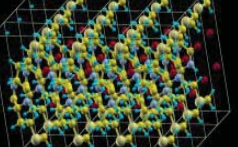 \\
\hline $\begin{array}{l}\text { 'cis- } \\
\text { cis' }\end{array}$ & & 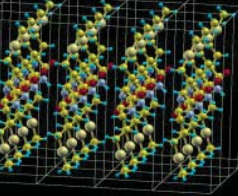 & 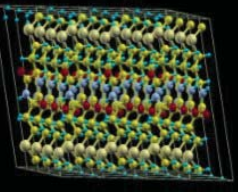 \\
\hline $\begin{array}{l}\text { 'tran } \\
\text { s-cis' }\end{array}$ & & & $x^{3}+y^{3}+t^{5}$ \\
\hline
\end{tabular}

Figure 5.3: Step II- Configurations of cyclo (S-Met-S-Met) crystals having different conformations of their two $\left(-\mathrm{CH}_{2}-\mathrm{CH}_{2}-\mathrm{S}_{-}-\mathrm{CH}_{3}\right)$ groups with $\phi_{\text {ring_ab }}=45^{\circ}$.

\subsubsection{Step-III: Refinement of the crystal structure}

After gaining a general picture of the relative orientation of the two side chains, now we focus on the 'up-down' conformation only with an aim to refine the rotational conformations of its side chains, since it is predicted to be energetically preferred. Considering the $s p^{3}$ hybridization of carbon atoms in an alkane chain (an example is given in Figure 5.4a for a two-carbon alkane chain), one would expect their bonding preference to be along the tetrahedral direction in a crystal. Thus, in particular, the next connecting carbon or sulfur atom can have one of the three orientations along its 
tetrahedral $\sigma$ bonds in such an alkane-like $\left(-\mathrm{CH}_{2}-\mathrm{CH}_{2}-\mathrm{S}_{-}-\mathrm{CH}_{3}\right)$ chain. This will lead to $3^{3}$ different conformers for a single chain. Since there are two cyclo $\left(-\mathrm{CH}_{2}-\mathrm{CH}_{2}-\mathrm{S}-\mathrm{CH}_{3}\right)$ chains in the cyclo (Met-Met) molecule, 729 kinds of unique molecular conformations are possible. However, based on our screening in Step I and II with the chemical intuition, it is not necessary to calculate all of the conformations, because some of these molecular conformations are likely to be energetically unstable due to high steric restrictions in a crystalline environment.

A flow chart to generate the conformers for the (S-Met-S-Met) crystal is given in Figures 5.4b-5.4e. One of the side chains of the (S-Met-S-Met) crystal takes a straightchain, paraffin-like conformation which is uniquely defined while the other side chain could have multiple conformations. In order to construct this second chain $\left(-\mathrm{CH}_{2}-\mathrm{CH}_{2}-\mathrm{S}-\right.$ $\mathrm{CH}_{3}$ ), positions of carbon and sulfur atoms along the chain need to be defined. Therefore, we start with defining the position of the first carbon $\mathrm{C} 1$ by introducing a $-\mathrm{CH}_{3}$ group connecting to a carbon atom on the ring $\mathrm{C} 4$ (Figure 5.4b). There is only one option for the $\mathrm{C} 1$ position. The dihedral angle $\mathrm{H} 12-\mathrm{C} 4-\mathrm{C} 1-\mathrm{H} 1$ is set to $180^{\circ}$ based on the minimum energy principle illustrated in Figure 5.4a. Next, we bring in the second carbon atom, C9. The C9 atom can take the orientation of either H5 or H14 (Figure 5.4c). We now bring the next $\mathrm{S} 2$ atom which has three choices (i.e. H14, $\mathrm{H} 15$ or H16) for the orientation along the tetrahedral directions. Among these choices, however, the orientation along H15 leaning upward is found to be energetically not preferred (step I) as it will limit packing of molecules in the crystalline form. So we are left with two choices (Figure 5.4d). 
Finally, we follow the possible tetrahedral orientations to bring in the third carbon C10 where the dihedral angle $\mathrm{C} 1-\mathrm{C} 9-\mathrm{S} 2-\mathrm{C} 10$ can be set to $60^{\circ}, 180^{\circ}$ or $-60^{\circ}$. Due to the steric effect, the $-60^{\circ}$ conformer is ruled out. Figure 6.4 e shows a choice of $60^{\circ}$. Overall, 8 distinct crystal conformations are generated for further electronic structure calculations (Figure 5.5).

a)
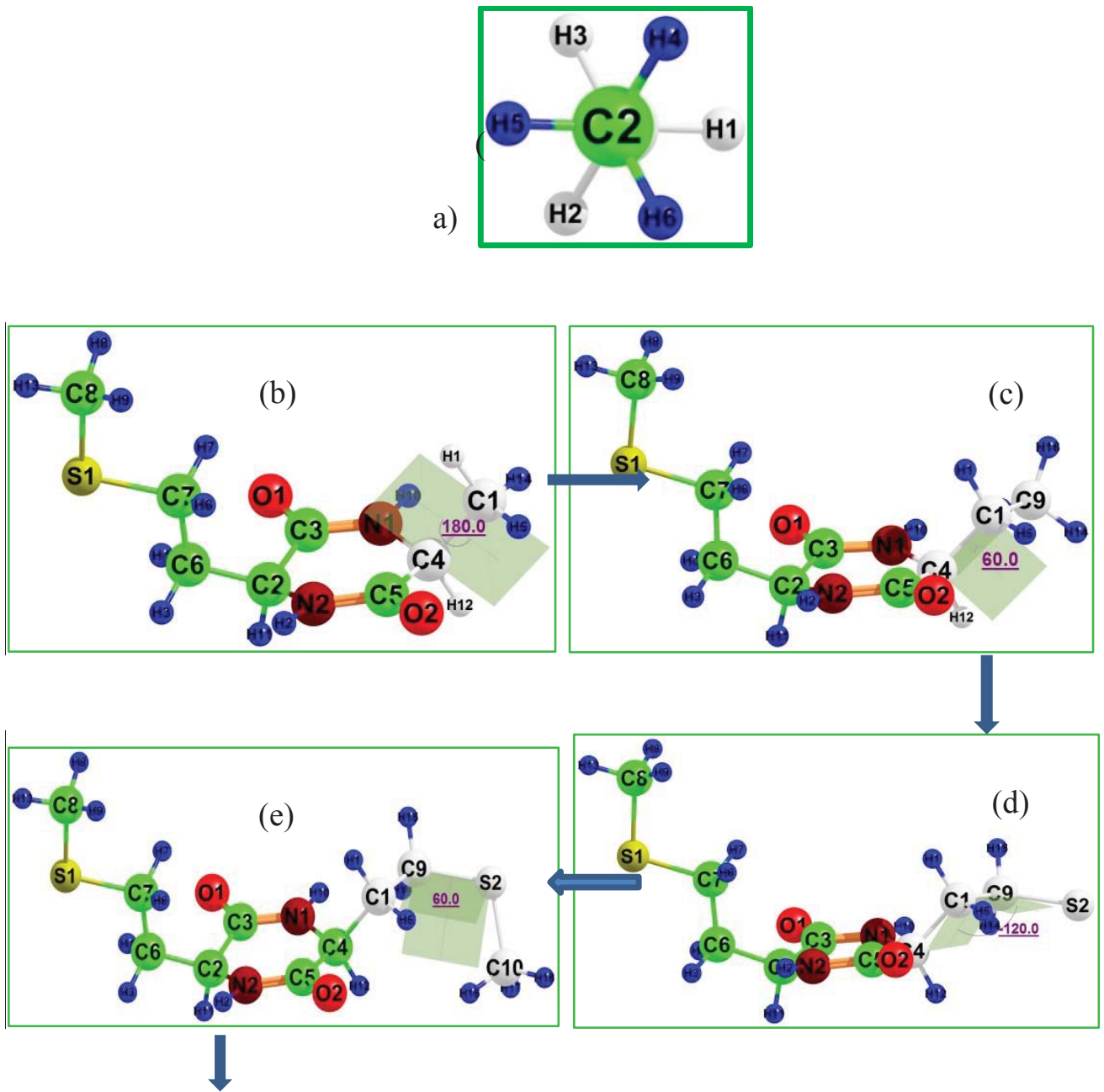

Conformation considered for full 
Figure 5.4: Step III- (a) Newman projection representation of the ethane molecule, (b)-(e) the flow chart showing the generation of the cyclo (S-Met-S-Met) conformers via rotation of the side chains. Atomic symbols: $\mathrm{C}$ in green, $\mathrm{N}$ in brown, $\mathrm{O}$ in red, $\mathrm{S}$ in golden yellow and $\mathrm{H}$ in blue.

Among the conformers considered, the ground state conformation in accordance with the principle of minimum energy is found to be the conformation ' 4 ' in Table 5.3.

Table 5.3: The structural parameters of the conformers of the (S-Met-S-Met) crystal.

\begin{tabular}{|c|c|c|c|c|c|c|c|c|c|}
\hline \multicolumn{2}{|l|}{ Conformers } & 1 & 2 & 3 & 4 & 5 & 6 & 7 & 8 \\
\hline \multicolumn{2}{|l|}{ Space group } & P1 & P1 & P1 & $\mathrm{P} 1$ & P1 & $\mathrm{P} 1$ & $\mathrm{P} 1$ & P1 \\
\hline \multicolumn{2}{|l|}{ Total energy } & -199.34 & -199.33 & -199.06 & -199.55 & -198.66 & -199.20 & -199.14 & -199.43 \\
\hline \multirow{3}{*}{ Lattice } & $\mathrm{a}$ & 4.62 & 4.75 & 5.36 & 4.76 & 4.69 & 8.29 & 4.77 & 4.74 \\
\hline & $\mathrm{b}$ & 5.19 & 5.06 & 7.28 & 4.86 & 5.16 & 4.84 & 5.08 & 5.12 \\
\hline & $\mathrm{c}$ & 13.18 & 13.21 & 10.13 & 13.02 & 12.96 & 8.67 & 12.77 & 12.84 \\
\hline parameters & $\alpha$ & 75.9 & 87.7 & 90.0 & 88.6 & 83.9 & 71.1 & 87.8 & 79.1 \\
\hline \multirow{2}{*}{$\left(\AA,^{\circ}\right)$} & $\beta$ & 81.6 & 70.8 & 83.6 & 94.7 & 78.2 & 105.2 & 79.5 & 82.2 \\
\hline & $\gamma$ & 76.5 & 76.9 & 55.4 & 78.8 & 75.4 & 78.5 & 74.9 & 75.6 \\
\hline \multicolumn{2}{|l|}{ Unit cell volume } & 296.7 & 292.2 & 322.4 & 295.2 & 294.1 & 296.6 & 302.3 & 293.4 \\
\hline \multicolumn{2}{|l|}{ Density $\left(\mathrm{g} / \mathrm{cm}^{3}\right)$} & 1.47 & 1.49 & 1.35 & 1.48 & 1.48 & 1.47 & 1.44 & 1.48 \\
\hline
\end{tabular}




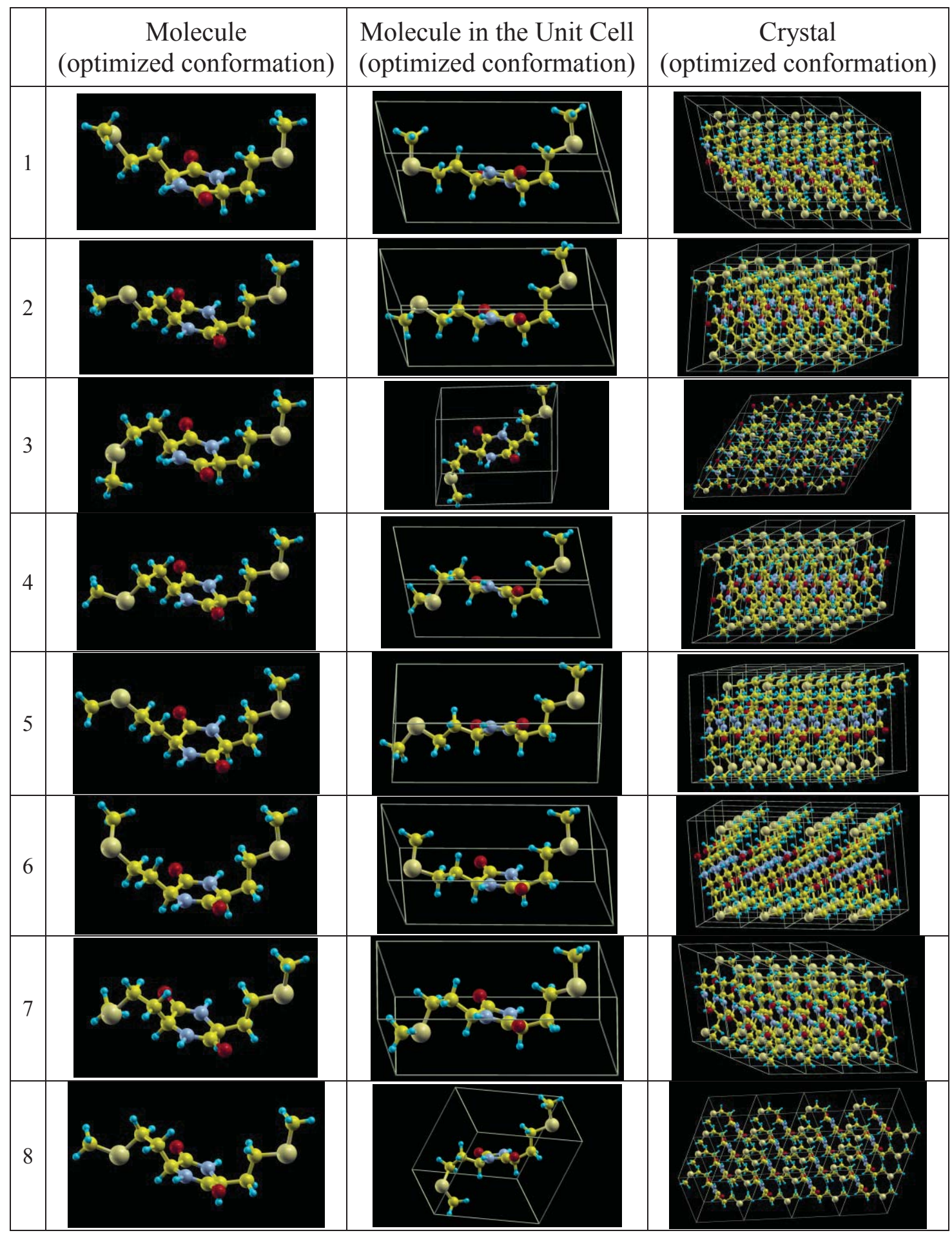


Figure 5.5: Step III- Refinement of the packing conformations of the cyclo (S-Met-S-Met) crystal.

\subsubsection{Comparison with experiments}

A comparison of the structural parameters of the ground state conformation with the corresponding experimental values is given in Table 5.4. We find that the predicted shape of the unit cell is in excellent agreement with the one obtained from experiments with deviations in angles to be less than $3^{\circ}$. All the calculated lattice constants $a, b$, and $c$ are predicted to be slightly smaller than the corresponding experimental values with differences of about $3 \%$.

Table 5.4: Comparison of calculated and experimental values of structural properties of the cyclo (S-Met-S-Met) and its enantiomer cyclo (R-Met-R-Met) crystals.

\begin{tabular}{|c|c|c|c|c|c|}
\hline & \multicolumn{3}{|c|}{ S-Met-S-Met } & \multirow{2}{*}{\begin{tabular}{|c|} 
R-Met-R-Met \\
This work \\
\end{tabular}} \\
\hline & & This work & $\begin{array}{l}\text { Experiment }^{[21]} \\
\qquad(100 \mathrm{~K})\end{array}$ & $\begin{array}{c}\text { Experiment }^{[20]} \\
(273 \mathrm{~K})\end{array}$ & \\
\hline \multicolumn{2}{|l|}{ Space group } & P1 & P1 & P1 & P1 \\
\hline \multirow{6}{*}{$\begin{array}{l}\text { Lattice parameters } \\
\qquad\left(\AA,^{\circ}\right)\end{array}$} & $\mathrm{a}$ & 4.74 & 4.903 & 4.885 & 4.74 \\
\hline & $\mathrm{b}$ & 5.12 & 5.252 & 5.304 & 5.11 \\
\hline & $\mathrm{c}$ & 12.84 & 13.231 & 13.469 & 12.86 \\
\hline & $\alpha$ & 79.1 & 78.65 & 74.2 & 100.9 \\
\hline & $\beta$ & 82.2 & 86.64 & 80.3 & 82.2 \\
\hline & $\gamma$ & 75.6 & 74.24 & 79.4 & 104.3 \\
\hline \multicolumn{2}{|l|}{ Unit cell volume } & 295.19 & 319.21 & 327.52 & 295.10 \\
\hline
\end{tabular}


It is worth noting that the lattice parameter $(c)$ along the molecular chain direction shows a sensitivity with temperature; the $c$ values are $13.231,13.469 \AA$ at $100 \mathrm{~K}$ and 273 $\mathrm{K}$, respectively. ${ }^{[20-21]}$ It is known that the organic crystals are generally distinguished from ionic or covalent crystals by their large thermal expansion coefficients. A large flexibility of the (S-Met-S-Met) molecule along the chain direction is the primary reason for the variation in $c$ in the crystalline phase considering that the so-called bending modes can be excited under $100 \mathrm{~K}$.

Under the harmonic approximation, the potential energy is a quadratic function of the bond length $R$ or the bond angle $\theta$ for stretching and bending vibrations, respectively.

$$
E=\frac{1}{2} k_{B}\left(R-R_{0}\right)^{2} \text { or } E=\frac{1}{2} k_{\theta}\left(\theta-\theta_{0}\right)^{2}
$$

Solving the Schrödinger wave equation, the energy states for each normal coordinate are given by

$$
E=\left(n+\frac{1}{2}\right) h v
$$

where $v$ is the vibrational frequency, $n$ is a quantum number describing the ground state $(n=0)$ and excited states $(n=1,2,3 \ldots)$.

Since the stretching frequencies (of about $2000-3000 \mathrm{~cm}^{-1}$ ) exceed by several times the bending ones (of several $100 \mathrm{~cm}^{-1}$ ), the excited states of stretching can only be accessible at $1000 \mathrm{~K}$, while the high order of bending modes can be excited under $100 \mathrm{~K}$. If we take 
the first excited state to be at $\mathrm{T}=100 \mathrm{~K}$ and the second excited state at $\mathrm{T}=273 \mathrm{~K}$, then the ratio of vibrational energy at $\mathrm{T}=0,100$ and $273 \mathrm{~K}$ is turned out to be $1: 3: 5$.

Since the square of oscillation amplitude is directly proportional to the vibrational energy, the vibration amplitude has a ratio of $1: \sqrt{3}: \sqrt{5}$ for $\mathrm{T}=0,100$ and $273 \mathrm{~K}$, respectively.

The following expressions can then be satisfied:

$$
\begin{aligned}
& c_{100 K}-c_{0 K}=(\sqrt{3}-1) c_{0} \\
& c_{273 K}-c_{0 K}=(\sqrt{5}-1) c_{0}
\end{aligned}
$$

where $c_{0}$ is the intrinsic lattice constant without taking into account vibrations, $c_{0 \mathrm{~K}}$, $c_{100 \mathrm{~K}}$, and $c_{273 \mathrm{~K}}$ represent the lattice constants at $\mathrm{T}=0,100$ and $273 \mathrm{~K}$, respectively.

Using the experimental values at $100 \mathrm{~K}$ and $273 \mathrm{~K}$, the estimated value of $c$ at $0 \mathrm{~K}$ is found to be $12.885 \AA$ which is in excellent agreement with the predicted value of $12.84 \AA$ at $0 \mathrm{~K}$.

A detailed comparison of the predicted and experimental characteristics of the threedimensional molecular structure within the unit cell is given in Tables 5.5, 5.6 and 5.7. The calculated results find the bond lengths to differ by less than $0.02 \AA$, the bond angles by less than $2^{\circ}$; and the dihedral angles by less than $5^{\circ}$. This excellent agreement thus shows the accuracy of our proposed approach in determining the crystalline structure of peptides. 
Table 5.5: Comparison of bond lengths $(\AA)$ for the cyclo (S-Met-S-Met) crystal.

\begin{tabular}{|c|c|c|c|c|c|c|c|}
\hline \multirow{2}{*}{ Bond } & \multicolumn{2}{|c|}{ (S-Met-S-Met) } & (R-Met-R-Met) & \multirow{2}{*}{ Bond } & \multicolumn{2}{|c|}{ (S-Met-S-Met) } & (R-Met-R-Met) \\
\cline { 7 - 8 } & This work & Expt. $^{[12]}$ & This work & & This work & Expt. ${ }^{[1]}$ & This work \\
\hline S1-C10 & 1.804 & 1.7900 & 1.804 & S2-C9 & 1.809 & 1.7913 & 1.809 \\
\hline S1-C6 & 1.817 & 1.8106 & 1.817 & S2-C8 & 1.820 & 1.8080 & 1.820 \\
\hline C5-C6 & 1.526 & 1.5181 & 1.526 & C7-C8 & 1.530 & 1.5244 & 1.530 \\
\hline C4-C5 & 1.535 & 1.5275 & 1.534 & C2-C7 & 1.526 & 1.5212 & 1.526 \\
\hline N1-C2 & 1.461 & 1.4732 & 1.462 & N2-C4 & 1.463 & 1.4651 & 1.463 \\
\hline C2-C3 & 1.516 & 1.5093 & 1.517 & C1-C4 & 1.524 & 1.5179 & 1.524 \\
\hline N1-C1 & 1.337 & 1.3169 & 1.337 & N2-C3 & 1.335 & 1.3125 & 1.335 \\
\hline O1-C1 & 1.255 & 1.2539 & 1.255 & O1-C3 & 1.258 & 1.2456 & 1.258 \\
\hline
\end{tabular}


Table 5.6: Comparison of bond angles $\left({ }^{\circ}\right)$ for the cyclo (S-Met-S-Met) crystal.

\begin{tabular}{|c|c|c|c|c|c|c|c|}
\hline \multirow{2}{*}{ Angle } & \multicolumn{2}{|c|}{ (S-Met-S-Met) } & \multirow{2}{*}{$\begin{array}{c}\text { (R-Met- } \\
\text { R-Met) } \\
\text { This } \\
\text { work }\end{array}$} & \multirow{2}{*}{ Angle } & \multicolumn{2}{|c|}{ (S-Met-S-Met) } & \multirow{2}{*}{$\begin{array}{c}\text { (R-Met- } \\
\text { R-Met) } \\
\text { This work }\end{array}$} \\
\hline & $\begin{array}{l}\text { This } \\
\text { work }\end{array}$ & Expt. ${ }^{[12]}$ & & & $\begin{array}{l}\text { This } \\
\text { work }\end{array}$ & Expt. ${ }^{[12]}$ & \\
\hline C6-S1-C10 & 98.9 & 100.29 & 99.3 & C8-S2-C9 & 99.8 & 100.76 & 99.8 \\
\hline S1-C6-C5 & 110.3 & 109.61 & 110.0 & $\mathrm{~S} 2-\mathrm{C} 8-\mathrm{C} 7$ & 114.0 & 115.25 & 114.0 \\
\hline C4-C5-C6 & 112.3 & 113.14 & 112.4 & $\mathrm{C} 2-\mathrm{C} 7-\mathrm{C} 8$ & 113.8 & 114.51 & 113.7 \\
\hline $\mathrm{N} 1-\mathrm{C} 2-\mathrm{C} 7$ & 111.2 & 111.12 & 111.2 & $\mathrm{~N} 2-\mathrm{C} 4-\mathrm{C} 5$ & 109.2 & 109.69 & 109.3 \\
\hline $\mathrm{C} 3-\mathrm{C} 2-\mathrm{C} 7$ & 112.3 & 112.58 & 112.4 & $\mathrm{C} 1-\mathrm{C} 4-\mathrm{C} 5$ & 112.8 & 112.99 & 112.9 \\
\hline $\mathrm{C} 1-\mathrm{N} 1-\mathrm{C} 2$ & 125.27 & 125.08 & 125.0 & C3-N2-C4 & 125.8 & 126.48 & 125.7 \\
\hline $\mathrm{N} 1-\mathrm{C} 1-\mathrm{C} 4$ & 117.5 & 118.11 & 117.4 & $\mathrm{~N} 2-\mathrm{C} 3-\mathrm{C} 2$ & 117.2 & 117.70 & 117.2 \\
\hline $\mathrm{N} 1-\mathrm{C} 2-\mathrm{C} 3$ & 111.3 & 110.26 & 111.3 & $\mathrm{~N} 2-\mathrm{C} 4-\mathrm{C} 1$ & 111.6 & 110.83 & 111.6 \\
\hline O1-C1-N1 & 122.6 & 122.65 & 122.6 & $\mathrm{O} 1-\mathrm{C} 1-\mathrm{C} 4$ & 119.9 & 119.24 & 120.0 \\
\hline $\mathrm{O} 2-\mathrm{C} 3-\mathrm{N} 2$ & 122.1 & 122.62 & 122.1 & $\mathrm{O} 2-\mathrm{C} 3-\mathrm{C} 2$ & 120.6 & 119.64 & 120.6 \\
\hline
\end{tabular}

[Bond angles are paired according to chemical correspondence.] 
Table 5.7: Comparison of torsion angles $\left({ }^{\circ}\right)$ for the cyclo (S-Met-S-Met) crystal.

\begin{tabular}{|c|c|c|c|c|c|c|c|}
\hline \multirow{2}{*}{$\begin{array}{c}\text { Torsion } \\
\text { angle }\end{array}$} & \multicolumn{2}{|c|}{ (S-Met-S-Met) } & \multirow{2}{*}{$\begin{array}{c}\begin{array}{c}\text { (R-Met-R- } \\
\text { Met) }\end{array} \\
\text { This work }\end{array}$} & \multirow{2}{*}{$\begin{array}{c}\text { Torsion } \\
\text { angle }\end{array}$} & \multicolumn{2}{|c|}{ (S-Met-S-Met) } & \multirow{2}{*}{$\begin{array}{c}\text { (R-Met-R- } \\
\text { Met) }\end{array}$} \\
\hline & $\begin{array}{l}\text { This } \\
\text { work }\end{array}$ & Expt. ${ }^{[12]}$ & & & $\begin{array}{l}\text { This } \\
\text { work }\end{array}$ & Expt. $^{[12]}$ & \\
\hline $\begin{array}{l}\text { C10-S1- } \\
\text { C6-C5 }\end{array}$ & -178.2 & -177.8 & 178.5 & $\begin{array}{c}\text { C9-S2-C8- } \\
\text { C7 }\end{array}$ & -63.8 & -69.4 & 64.4 \\
\hline $\begin{array}{l}\text { C4-C5- } \\
\text { C6-S1 }\end{array}$ & -164.5 & -164.6 & 164.1 & $\begin{array}{l}\text { C2-C7- } \\
\text { C8-S2 }\end{array}$ & -57.9 & -59.4 & 58.2 \\
\hline $\begin{array}{l}\text { C1-C4- } \\
\text { C5-C6 }\end{array}$ & -53.2 & -52.3 & 53.4 & $\begin{array}{l}\text { C3-C2- } \\
\text { C7-C8 }\end{array}$ & 160.0 & 163.9 & -159.5 \\
\hline $\begin{array}{l}\text { C2-N1- } \\
\text { C1-C4 }\end{array}$ & 5.7 & 9.6 & -5.8 & $\begin{array}{l}\text { C4-N2- } \\
\text { C3-C2 }\end{array}$ & 0.1 & 1.8 & -0.6 \\
\hline $\begin{array}{l}\mathrm{N} 1-\mathrm{C} 1- \\
\mathrm{C} 4-\mathrm{N} 2\end{array}$ & 21.7 & 19.1 & -22.2 & $\begin{array}{l}\mathrm{N} 1-\mathrm{C} 2- \\
\mathrm{C} 3-\mathrm{N} 2\end{array}$ & 26.8 & 26.1 & -26.9 \\
\hline $\begin{array}{l}\text { C3-N2- } \\
\text { C4-C1 }\end{array}$ & -25.0 & -25.5 & 25.9 & $\begin{array}{l}\mathrm{C} 1-\mathrm{N} 1- \\
\mathrm{C} 2-\mathrm{C} 3\end{array}$ & -30.5 & -32.9 & 30.9 \\
\hline $\begin{array}{l}\text { C1-N1- } \\
\text { C2-C7 }\end{array}$ & -156.6 & -158.4 & 157.1 & $\begin{array}{l}\mathrm{C} 3-\mathrm{N} 2- \\
\mathrm{C} 4-\mathrm{C} 5\end{array}$ & -150.5 & -150.9 & 151.6 \\
\hline $\begin{array}{l}\text { C7-C2- } \\
\text { C3-N2 }\end{array}$ & 152.3 & 150.8 & -152.5 & $\begin{array}{l}\text { N1-C1- } \\
\text { C4-C5 }\end{array}$ & 145.2 & 142.7 & -145.9 \\
\hline $\begin{array}{c}\text { N2-C4- } \\
\text { C5-C6 }\end{array}$ & 71.6 & 71.9 & -71.4 & $\begin{array}{l}\text { N1-C2- } \\
\text { C7-C8 }\end{array}$ & -74.4 & -71.9 & 74.9 \\
\hline $\begin{array}{l}\text { C2-N1- } \\
\text { C1-O1 }\end{array}$ & -174.0 & -170.1 & 173.9 & $\begin{array}{l}\text { C4-N2- } \\
\text { C3-O2 }\end{array}$ & -177.0 & -175.7 & 176.4 \\
\hline $\begin{array}{l}\text { N1-C2- } \\
\text { C3-O2 }\end{array}$ & -156.0 & -156.3 & 156.0 & $\begin{array}{l}\text { N2-C4- } \\
\text { C1-O1 }\end{array}$ & -158.5 & -160.4 & 158.1 \\
\hline $\begin{array}{l}\mathrm{C} 7-\mathrm{C} 2- \\
\mathrm{C} 3-\mathrm{O} 2\end{array}$ & -30.6 & -31.6 & 30.4 & $\begin{array}{l}\mathrm{O} 1-\mathrm{C} 1- \\
\mathrm{C} 4-\mathrm{C} 5\end{array}$ & -35.0 & -36.8 & 34.5 \\
\hline
\end{tabular}

[Torsion angles are paired according to chemical correspondence.] 


\subsubsection{The enantiomer - cyclo (R-Met-R-Met) crystal}

Chiral materials are incongruent with their mirror images. The most significant manifestation of chirality is the appearance of the left- and right-handed molecules. The cyclo (Met-Met) molecule has two asymmetric carbon atoms and two chiral centers. Following the procedure outlined above, we have obtained its crystal structure by taking it to be mirror symmetric with the cyclo (S-Met-S-Met) (Figure 5.6).

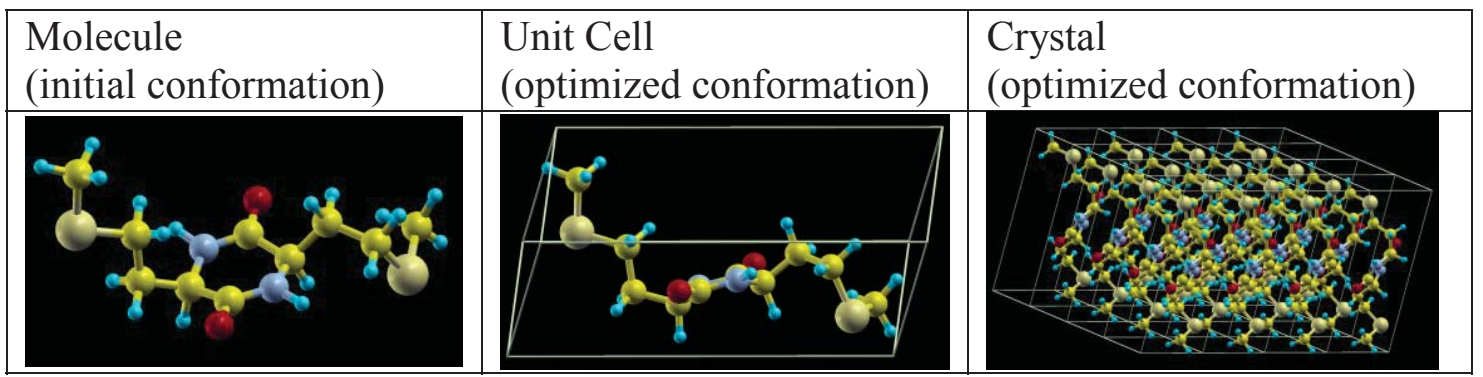

Figure 5.6: The molecular and crystalline structures of the cyclo (R-Met-R-Met). Atomic symbols: $\mathrm{C}$ in yellow, $\mathrm{N}$ in navy blue, $\mathrm{O}$ in red, $\mathrm{S}$ in gray and $\mathrm{H}$ in light blue.

As shown in Table 5.4, both (S-Met-S-Met) and (R-Met-R-Met) crystals have nearly the same lattice parameters (difference $<0.02 \AA$ ). The angles, $\alpha$ and $\gamma$ in the (R-Met-RMet) crystal are complementary to the corresponding angles in the (S-Met-S-Met) crystals, thus showing the mirror symmetry. Likewise the internal molecular configuration in the unit cell of the (R-Met-R-Met) matches exactly with that of the (SMet-S-Met) crystal, except for the dihedral angles. Due to the mirror symmetry, the dihedral angles differ by the sign having the same magnitude. Since no experimental data is available for the cyclo (R-Met-R-Met) crystal, we use the symmetry to infer its crystal 
structure which is shown in Figure 5.7. The predicted crystal structure is found to be very close to the inferred crystal structure for the cyclo (R-Met-R-Met) crystal.

\section{Experiment Theory}

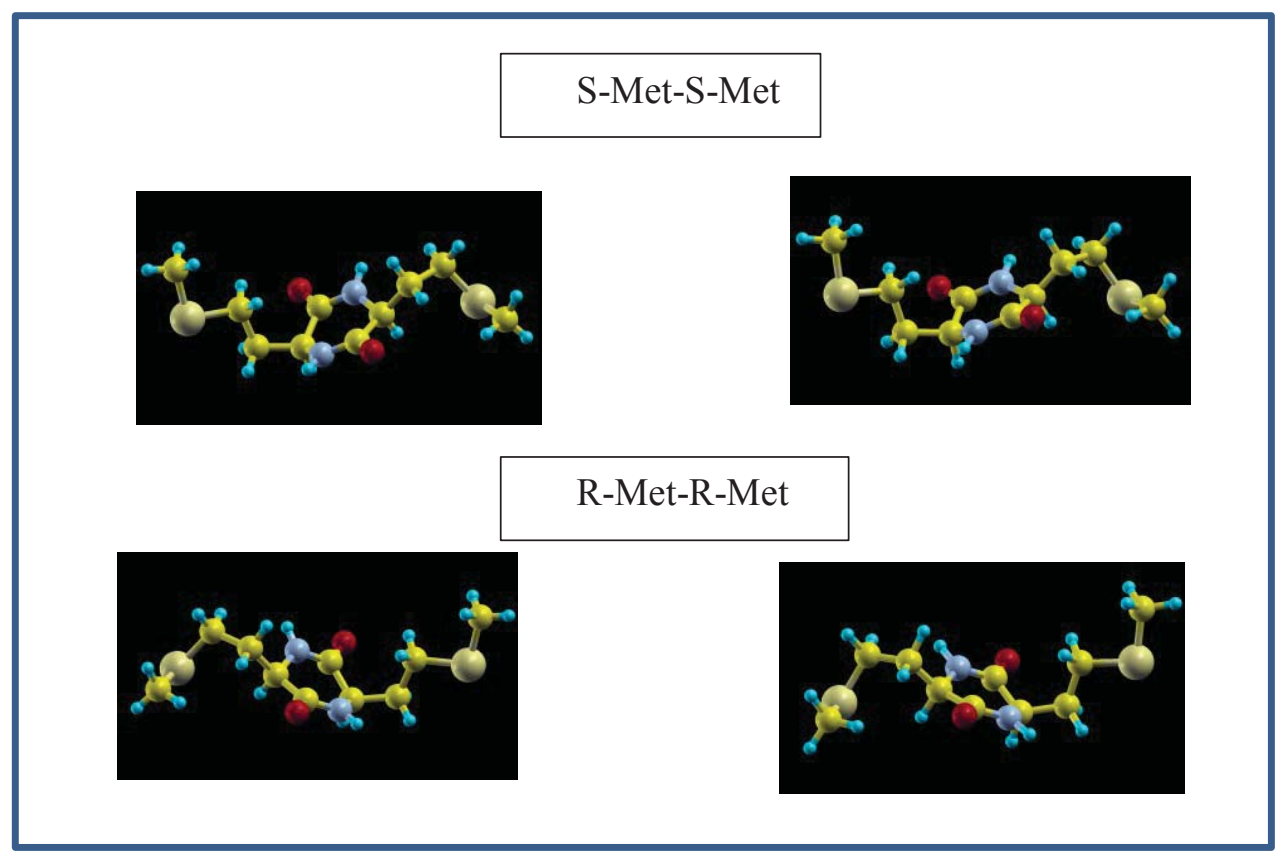

Figure 5.7: The ground state conformations of cyclo (S-Met-S-Met) and (R-Met-R-Met) crystals.

The electronic structure of a material depends on its structure and atomic compositions. The R/S enantiomers share mirror symmetry in their 3-D structures. Thus, the same band structure and electronic density of states were computed for the R/S enantiomer crystals (Figure 5.8) as an indicator of their basic physical and chemical properties in a non-chiral environment. 

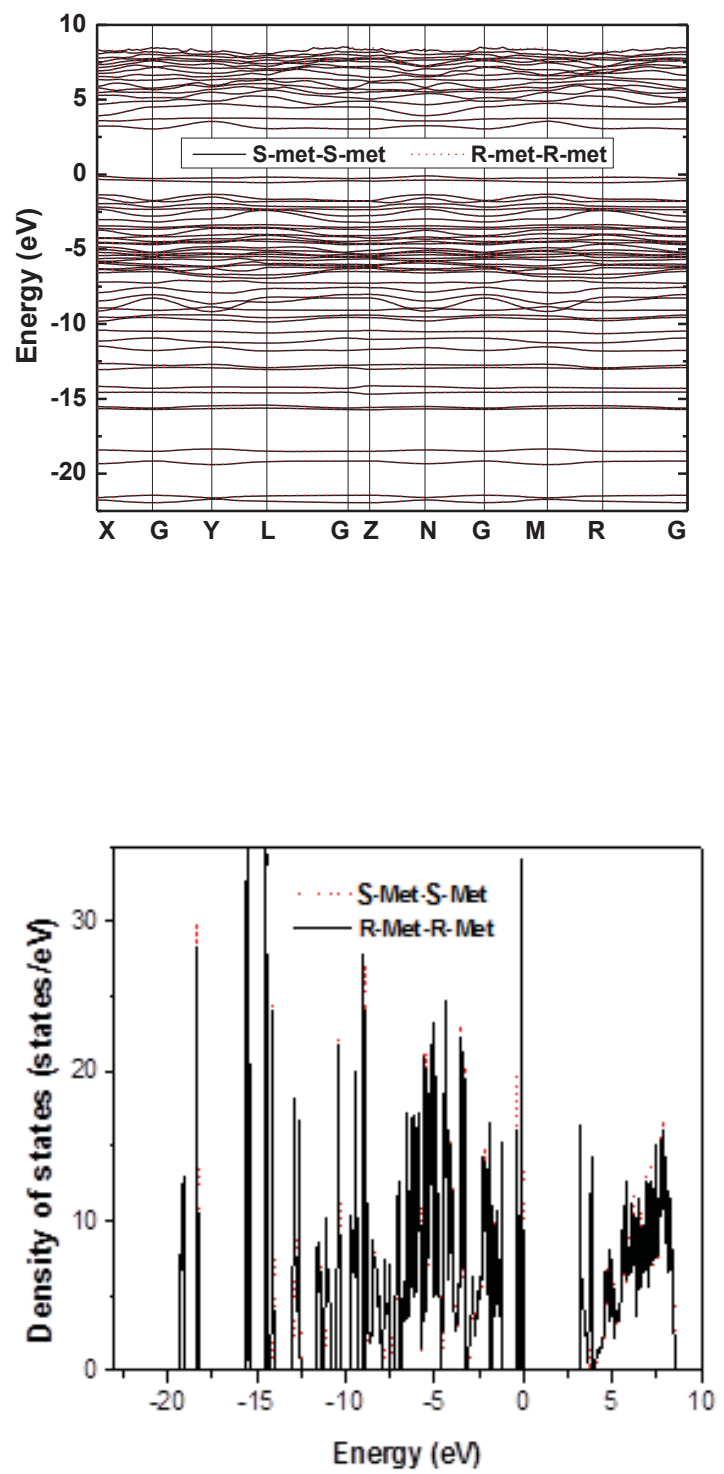

Figure 5.8: The calculated band structures and electronic density of states for the cyclo (S-Met-SMet) and (R-Met-R-Met) crystals. Zero of the energy is aligned to top of the valence band.

Figure 5.8 shows the calculated band structure and electronic density of states of both chiral crystals along the high symmetric k-point axis, $X-\Gamma-Y-L-\Gamma-Z-N-\Gamma-M-R-\Gamma$. 
The energy dispersions of bands for both crystals are small, and the uppermost valence band is formed by $\mathrm{S} 3 p$ states, with a width of about $0.45 \mathrm{eV}$. It is followed by a band primarily composed of $\mathrm{O} 2 p$ and $\mathrm{N} 2 p$ states. A wide band with the width of 6.45 $\mathrm{eV}$ is a composed of bands from $\mathrm{C} 2 \mathrm{p}, \mathrm{N} 2 p, \mathrm{O} 2 p$ and $\mathrm{S} 3 p$ states. The calculated band gap is $3.04 \mathrm{eV}$ at the GGA-DFT level of theory. It is expected that the predicted nature and location of the integrand transitions will assist the experimentalists in the interpretation of the optical spectra obtained by either reflectance or photoelectron spectroscopy.

\subsection{Summary}

In summary, the crystal structures of the cyclo (Met-Met) R/S enantiomer peptides are predicted using the method based on dispersion-corrected density functional theory. The excellent agreement of the calculated results with the corresponding experimental results for the cyclo (S-Met-S-Met) crystal demonstrates the accuracy and reliability of the proposed computational scheme. This approach determines both the lattice parameters and the molecular three-dimensional structure in the unit cell, and finds an accurate calculation of packing orientation of the molecule in the crystalline environment to be essential. Furthermore, the use of symmetry manipulation together with the knowledge of structural chemistry and chemical bonding reduce the number of probable conformers from 729 to 11 for the cyclo (Met-Met) crystals. It therefore facilitates sampling of the conformation space by first principles method with the modest computing resources. 
The proposed computational approach has many advantages. First of all, this approach enables us to explore the possibility of obtaining certain molecular crystals and further locating the most stable molecular crystal structure with the most accurate first-principles method for only modest cost. Secondly, it can not only obtain the 3-D structural information, but also other physical and chemical properties which can directly be compared with experimental measurements. And thirdly, this approach has been demonstrated to be very successful for a highly flexible peptide molecule. Efforts are currently taken to apply it to other cyclo-dipeptides class of compounds. 


\section{References}

[1].Fischer, C. C.; Tibbetts, K. J.; Morgan, D.; Ceder, G. Predicting crystal structure by merging data mining with quantum mechanics. Nat Mater, 5 (8), (2006) 641-646.

[2].Wang, Y. C.; Lv, J. A.; Zhu, L.; Ma, Y. M. Crystal structure prediction via particle-swarm optimization. Phys Rev B, 82 (9), (2010) 094116.

[3].Curtarolo, S.; Morgan, D.; Persson, K.; Rodgers, J.; Ceder, G. Predicting crystal structures with data mining of quantum calculations. Phys Rev Lett 91 (13), (2003) 135503.

[4].Podeszwa, R.; Rice, B. M.; Szalewicz, K. Crystal structure prediction for cyclotrimethylene trinitramine (RDX) from first principles. Phys Chem Chem Phys 11 (26), (2009) 5512-5518.

[5].Chan, H. C. S.; Kendrick, J.; Leusen, F. J. J. Molecule VI, a Benchmark CrystalStructure-Prediction Sulfonimide: Are Its Polymorphs Predictable? Angew Chem Int Edit, 50 (13), (2011) 2979-2981.

[6].Issa, N.; Karamertzanis, P. G.; Welch, G. W. A.; Price, S. L. Can the Formation of Pharmaceutical Cocrystals Be Computationally Predicted? I. Comparison of Lattice Energies. Cryst Growth Des, 9 (1), (2009) 442-453.

[7].Karamertzanis, P. G.; Kazantsev, A. V.; Issa, N.; Welch, G. W. A.; Adjiman, C. S.; Pantelides, C. C.; Price, S. L. Can the Formation of Pharmaceutical Cocrystals Be 
Computationally Predicted? 2. Crystal Structure Prediction. J Chem Theory Comput 5 (5), (2009) 1432-1448.

[8].Asmadi, A.; Neumann, M. A.; Kendrick, J.; Girard, P.; Perrin, M. A.; Leusen, F. J. J. Revisiting the Blind Tests in Crystal Structure Prediction: Accurate Energy Ranking of Molecular Crystals. J Phys Chem B 113 (51), (2009) 16303-16313.

[9].King, M. D.; Blanton, T. N.; Misture, S. T.; Korter, T. M. Prediction of the Unknown Crystal Structure of Creatine Using Fully Quantum Mechanical Methods. Cryst Growth Des, 11 (12), (2011)5733-5740.

[10].Kendrick, J.; Leusen, F. J. J.; Neumann, M. A.; van de Streek, J. Progress in Crystal Structure Prediction. Chem-Eur J, 17 (38), (2011) 10736-10744.

[11].Lund, A. M.; Orendt, A. M.; Pagola, G. I.; Ferraro, M. B.; Facelli, J. C. Optimization of Crystal Structures of Archetypical Pharmaceutical Compounds: A PlaneWave DFT-D Study Using Quantum Espresso. Cryst Growth Des, 13 (5), (2013) 21812189.

[12].Lehmann, C. W. Crystal Structure Prediction-Dawn of a New Era. Angew Chem Int Edit, 50 (25), (2011) 5616-5617.

[13].Gavezzotti, A. Are Crystal-Structures Predictable. Accounts Chem Res, 27 (10), (1994) 309-314. 
[14].Lommerse, J. P. M.; Motherwell, W. D. S.; Ammon, H. L.; Dunitz, J. D.; Gavezzotti, A.; Hofmann, D. W. M.; Leusen, F. J. J.; Mooij, W. T. M.; Price, S. L.; Schweizer, B.; Schmidt, M. U.; van Eijck, B. P.; Verwer, P.; Williams, D. E. A test of crystal structure prediction of small organic molecules. Acta Crystallogr B, 56, (2000) 697-714.

[15].Motherwell, W. D. S.; Ammon, H. L.; Dunitz, J. D.; Dzyabchenko, A.; Erk, P.; Gavezzotti, A.; Hofmann, D. W. M.; Leusen, F. J. J.; Lommerse, J. P. M.; Mooij, W. T. M.; Price, S. L.; Scheraga, H.; Schweizer, B.; Schmidt, M. U.; van Eijck, B. P.; Verwer, P.; Williams, D. E. Crystal structure prediction of small organic molecules: a second blind test. Acta Crystallogr B, 58, (2002) 647-661.

[16].Day, G. M.; Motherwell, W. D. S.; Ammon, H. L.; Boerrigter, S. X. M.; Della Valle, R. G.; Venuti, E.; Dzyabchenko, A.; Dunitz, J. D.; Schweizer, B.; van Eijck, B. P.; Erk, P.; Facelli, J. C.; Bazterra, V. E.; Ferraro, M. B.; Hofmann, D. W. M.; Leusen, F. J. J.; Liang, C.; Pantelides, C. C.; Karamertzanis, P. G.; Price, S. L.; Lewis, T. C.; Nowell, H.; Torrisi, A.; Scheraga, H. A.; Arnautova, Y. A.; Schmidt, M. U.; Verwer, P. A third blind test of crystal structure prediction. Acta Crystallogr B, 61, (2005) 511-527.

[17].Day, G. M.; Cooper, T. G.; Cruz-Cabeza, A. J.; Hejczyk, K. E.; Ammon, H. L.; Boerrigter, S. X. M.; Tan, J. S.; Della Valle, R. G.; Venuti, E.; Jose, J.; Gadre, S. R.; Desiraju, G. R.; Thakur, T. S.; van Eijck, B. P.; Facelli, J. C.; Bazterra, V. E.; Ferraro, M. B.; Hofmann, D. W. M.; Neumann, M. A.; Leusen, F. J. J.; Kendrick, J.; Price, S. L.; 
Misquitta, A. J.; Karamertzanis, P. G.; Welch, G. W. A.; Scheraga, H. A.; Arnautova, Y. A.; Schmidt, M. U.; van de Streek, J.; Wolf, A. K.; Schweizer, B. Significant progress in predicting the crystal structures of small organic molecules - a report on the fourth blind test. Acta Crystallogr B, 65, (2009) 107-125.

[18].Bardwell, D. A.; Adjiman, C. S.; Arnautova, Y. A.; Bartashevich, E.; Boerrigter, S. X. M.; Braun, D. E.; Cruz-Cabeza, A. J.; Day, G. M.; Della Valle, R. G.; Desiraju, G. R.; van Eijck, B. P.; Facelli, J. C.; Ferraro, M. B.; Grillo, D.; Habgood, M.; Hofmann, D. W. M.; Hofmann, F.; Jose, K. V. J.; Karamertzanis, P. G.; Kazantsev, A. V.; Kendrick, J.; Kuleshova, L. N.; Leusen, F. J. J.; Maleev, A. V.; Misquitta, A. J.; Mohamed, S.; Needs, R. J.; Neumann, M. A.; Nikylov, D.; Orendt, A. M.; Pal, R.; Pantelides, C. C.; Pickard, C. J.; Price, L. S.; Price, S. L.; Scheraga, H. A.; van de Streek, J.; Thakur, T. S.; Tiwari, S.; Venuti, E.; Zhitkov, I. K. Towards crystal structure prediction of complex organic compounds - a report on the fifth blind test. Acta Crystallogr B, 67, (2011)535-551.

[19]. Prasad, C. Bioactive Cyclic Dipeptides. Peptides, 16 (1), (1995) 151-164.

[20].Valle, G.; Guantieri, V.; Tamburro, A. M. On the Molecular and CrystalStructure of Cyclo(S-Methionyl-S-Methionyl). J Mol Struct, 220, (1990) 19-24.

[21].Mendham, A. P.; Dines, T. J.; Snowden, M. J.; Withnall, R.; Chowdhry, B. Z. IR/Raman spectroscopy and DFT calculations of cyclic di-amino acid peptides. Part III: comparison of solid state and solution structures of cyclo(L-Ser-L-Ser). $J$ Raman Spectrosc, 40 (11), (2009) 1508-1520. 
[22].Kresse, G.; Furthmüller, J. Efficient iterative schemes for ab initio total-energy calculations using a plane-wave basis set. Physical Review B, 54 (16), (1996) 1116911186.

[23].Grimme, S. Semiempirical GGA-type density functional constructed with a longrange dispersion correction. J Comput Chem, 27 (15), (2006) 1787-1799.

[24].Blochl, P. E. Projector Augmented-Wave Method. Phys Rev B, 50 (24), (1994) 17953-17979.

[25].Kresse, G.; Joubert, D. From ultrasoft pseudopotentials to the projector augmented-wave method. Physical Review B, 59 (3), (1999) 1758-1775.

[26].Perdew, J. P.; Chevary, J. A.; Vosko, S. H.; Jackson, K. A.; Pederson, M. R.; Singh, D. J.; Fiolhais, C. Atoms, Molecules, Solids, and Surfaces - Applications of the Generalized Gradient Approximation for Exchange and Correlation. Phys Rev B, 46 (11), (1992) 6671-6687. 


\section{Chapter 6}

\section{Molecular Dynamics Method}

\subsection{Introduction}

First-principles methods based on principles of quantum mechanics are now routinely used to study atoms, molecules, and solids, but they are limited to a few hundred atoms a limit imposed by the availability of computational resources. In addition to that, the effect of temperature is generally not included as most of the electronic structure calculations are performed in the framework of static lattice approximation. The study of biological macromolecules ${ }^{[1-6]}$, on the other hand, requires simulation of a few thousand atoms in an aqueous environment at finite temperatures to attain a satisfactory comparison with experiments. Such a study is then performed by atomistic methods based on molecular dynamics (MD) which can include the effect of temperature and environment satisfactorily. MD approaches can describe macromolecules as composed of nuclei and electrons moving under the action of the potential field in accordance with Newton's law. The macroscopic properties of a given system can then be obtained by an analysis of its particles trajectories following the methods of statistical physics. 


\subsection{Molecular force fields}

The theoretical basis of molecular dynamics is based on Newtonian mechanics. The equation of motion of each atom can be written as:

$$
m_{i} \frac{\partial r_{i}^{2}}{d t^{2}}=f_{i} \quad f_{i}=-\frac{\partial}{\partial r_{i}} U_{i}
$$

Where $m_{\mathrm{i}}, r_{\mathrm{i}}$, and $U_{\mathrm{i}}$ are the mass, position, and potential energy of the $i^{\text {th }}$ atom, respectively; $f_{\mathrm{i}}$ is the force on the $i^{\text {th }}$ atom.

Knowing the potential energy associated with atoms, one can calculate force acting on each atom of the system. Using the initial position and the initial velocity, one can easily calculate trajectory of each atom of the system. Therefore, a satisfactory description of the potential energy term of the system is a key step in MD simulations. Note that the potential energy term of a system can be written in terms of bonded, non-bonded and external components.

Non-bonded potential energy term $\left(U_{\text {non-bonded }}\right)$ represents the so-called non-bonded interactions between atoms, and can be split into 1-body, 2-body, 3-body . . . terms as follows:

$$
U_{\text {non-bonded }}=\sum_{i} u\left(r_{i}\right)+\sum_{i} \sum_{j>i} v\left(r_{i}, r_{j}\right)+\cdots
$$


Here $u\left(r_{i}\right)$ represents the self-energy of the $i^{\text {th }}$ atom in the system, and $v\left(r_{\mathrm{i}}, r_{\mathrm{j}}\right)$ the pairwise interaction energy between atoms in the system. Ignoring the higher order terms, one can describe $U^{\text {pair }}{ }_{\text {non-bonded }}$ in terms of Coulombic, van der Waals, and hydrogen bond terms. The van der Waals interaction term is generally described by the Lennard-Jones potential $^{[7]}$ :

$$
U_{v d w}=\varepsilon\left[\left(\frac{\sigma}{r}\right)^{12}-\left(\frac{\sigma}{r}\right)^{6}\right]
$$

As a function of the interatomic distance $r$ with two parameters $\varepsilon$ and $\sigma . \varepsilon$ is the depth of the potential well, $\sigma$ is the finite distance at which derivative of the interatomic potential is zero.

The Coulomb term can be expressed as:

$$
U_{q}=\frac{q_{1} q_{2}}{4 \pi \varepsilon r}
$$

Where $q_{1}, q_{2}$ are the charges and $\varepsilon$ is the permittivity of space.

Considering that a large number of hydrogen bonds exist in a biological macromolecule, the hydrogen bonding terms are expressed as ${ }^{[8]}$ :

$$
U_{H}=\left(\frac{A}{r^{m}}-\frac{B}{r^{n}}\right)(\cos \theta)^{p}
$$


Where $A$ and $B$ are constants; $r$ is distance between two chemical functional groups; and $(\cos \theta)^{p}$ is a control function.

In a molecule, the role of bonding terms describing interactions between the adjacent atoms is obviously important. The force field is generally approximated in terms of

$$
U_{\text {bonded }}=U_{s}+U_{a}+U_{t}+U_{o}
$$

where $U_{\mathrm{s}}$ is the bond stretching potential energy, $U_{\mathrm{a}}$ is the bond angle bending potential energy, $U_{\mathrm{t}}$ is the dihedral angle distortion potential energy, and $U_{\mathrm{o}}$ is the dihedral angle vibrational potential energy.

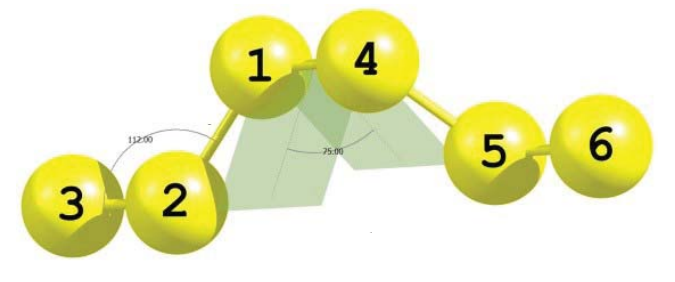

Figure 6.1: Geometry of a simple chain molecule illustrating the bond distance $\boldsymbol{R}_{45}$, bond angel $\theta_{123}$, and torsion angle $\varphi_{2145}$

The vibrational energy which takes into account the movement of an atom along its equilibrium position can be expressed as:

$$
U_{s}=k_{2}\left(r-r_{0}\right)^{2}+k_{3}\left(r-r_{0}\right)^{3}+k_{4}\left(r-r_{0}\right)^{4}
$$


The higher-order polynomial terms (the second and third terms in eqn. 6.8) are generally neglected, and $U_{\mathrm{s}}$ can be simplified as:

$$
U_{s}=K_{b}\left(b-b_{0}\right)^{2}
$$

$K_{b}$ is the spring constant of the bond and $b_{0}$ is the bond length at the equilibrium.

In order to include the temperature effect on rotation of bonds, we write:

$$
U_{s}=k_{2}\left(\theta-\theta_{0}\right)^{2}+k_{3}\left(\theta-\theta_{0}\right)^{3}+k_{4}\left(\theta-\theta_{0}\right)^{4}
$$

Similarly, the higher-order terms (the second and third terms in eqn. 6.10) can safely be removed and $U_{\mathrm{s}}$ is left as:

$$
U_{s}=\frac{1}{2} K_{\theta}\left(\theta-\theta_{0}\right)^{2}
$$

Where $K_{\theta}$ is the spring constant of the bond angle and $\theta_{0}$ is the bond angle at the equilibrium.

In molecules, rotation of bonds due to temperature effects will cause distortions in the molecular skeleton leading to the dihedral angle energy term. The dihedral term depends on how the bonds are connected (Figure 6.2). 
Figure 6.2: A ball and stick model of a molecule showing (a) torsion type, and (b) inversion type bond connections.
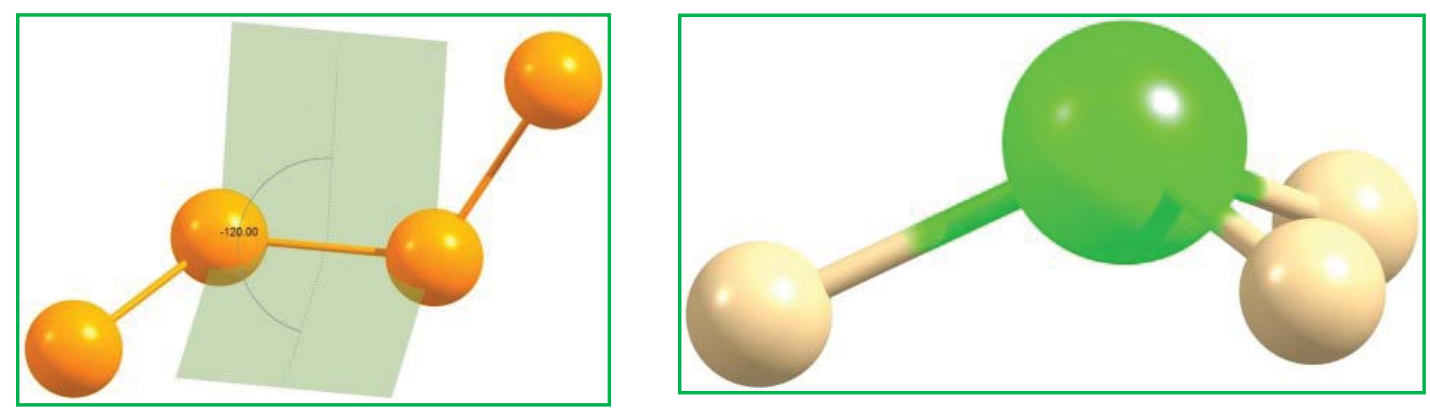

The Torsion type connection is given by:

$$
U_{\text {torsion }}=K_{\varphi}(1+\cos (n \varphi-\delta))
$$

where $K_{\varphi}$ is the spring constant of the dihedral angle $\varphi ; \delta$ is the dihedral angle at equilibrium state.

Also, the Inversion type connection is given by:

$$
U_{s}=\frac{1}{2} K_{\xi}\left(\xi-\xi_{0}\right)^{2}
$$

$K_{\xi}$ is the spring constant of the inversion term and $\xi$ is the inversion angle.

In summary, a force field model to describe interactions within a molecule can be written as: 


$$
\begin{aligned}
& V_{i}(r)=V_{i}\left(r_{1}, r_{2} \ldots . . r_{N}\right) \\
& =\sum_{b} \frac{1}{2} K_{b}\left(b-b_{0}\right)^{2}+\sum_{\theta} \frac{1}{2} K_{\theta}\left(\theta-\theta_{0}\right)^{2}+\sum_{\xi} \frac{1}{2} K_{\xi}\left(\xi-\xi_{0}\right)^{2} \\
& +\sum_{\varphi} K_{\varphi}[1+\operatorname{Cos}(n \varphi-\delta)]+\sum_{i<j}\left[\frac{C_{12}}{r_{i j}^{12}}-\frac{C_{6}}{r_{i j}^{6}}-\frac{q_{i} q_{j}}{4 \pi \varepsilon_{0} \varepsilon_{j} r_{i j}}\right]
\end{aligned}
$$

Eq. (6.14) is a CHARMM type force field form ${ }^{[9]}$. Some of molecular mechanics force-fields, e.g. MM3 ${ }^{[10-12]}$ and MM4 ${ }^{[13-15]}$ include many cross-terms. For a large system consisting of a few thousand atoms (e.g. proteins), relatively simple force fields, such as AMBER ${ }^{[16,17]}$ and OPLS ${ }^{[18]}$ are also available. The functional forms of AMBER and OPLS are determined by quantum chemical calculations combined with thermo physical and phase coexistence data.

Having specified the potential energy function $U\left(r^{N}\right)$, the next step is to calculate the atomic forces:

$$
f_{i}=-\frac{\partial}{\partial r_{i}} U\left(r^{N}\right)
$$

\subsection{Algorithm}

A series of detailed atomic positions in the system is obtained by solving the differential Newton's equation.

$$
a_{i}=\frac{d^{2} r_{i}}{d t^{2}}=\frac{f_{i}}{m_{i}}=-\frac{\partial}{\partial r_{i}} U\left(r^{N}\right) \cdot \frac{1}{m}
$$


Considering that the system consists of $\mathrm{N}$ particles, one needs to describe their movements by numerical integration of $3 \mathrm{~N}$ equations of motions. Such a task is performed by the discrete-time approach using Taylor expansion expression for the next step of position and velocity from the current position, velocity and acceleration.

$$
\begin{gathered}
r_{n+1}=r_{n}+v_{n} \Delta t+\frac{1}{2}\left(\frac{f_{n}}{m}\right) \Delta t^{2}+\frac{1}{3 !} \frac{d^{3} r_{n}}{d t^{3}}+O\left(\Delta t^{4}\right) \\
v_{n+1}=v_{n}+a_{n} \Delta t+\frac{1}{2} \frac{d^{2} v_{n}}{d t^{2}}+O\left(\Delta t^{3}\right)
\end{gathered}
$$

Since the integrator is a second order equation, we need two initial conditions to start the integral calculation; the initial position and initial particle velocity are considered to be known quantities. The approach will then be taken as follows: (i) the force on the each of atoms can be calculated by the initial position and the force field. Using these forces one can calculate acceleration of each atom; (ii) the position of each atom for next time can be calculated using Eq.6.17 by the initial position, initial velocity and acceleration which have already gotten in the step 1; (iii) using the Eq. 6.17 and some methods can calculate the velocity of each atom for next time; (iv) now using the new position and velocity of each atom to repeat from step 1 to step 3, one can get the quantities for the third time step. The whole cycle repeats. In this way, we can get the whole trajectories of atoms after a certain amount of time.

The rule of thumb is to select $\Delta t$ less than the atomic vibration period, which is of the order of $10^{-15}$ second. It should be noted that if the chosen $\Delta t$ is not appropriate, the 
particle trajectories may quickly move away from their true path. Furthermore, the choice of algorithm is very important for obtaining valid data from MD simulations. There are four algorithms, namely Verlet algorithm ${ }^{[22]}$, velocity-Verlet method ${ }^{[23]}$, leap-Frog

method ${ }^{[24]}$, and predictor-corrector method ${ }^{[25,26]}$ which are most commonly employed in MD simulations.

\subsection{Initial conditions}

The initial conditions of a molecular dynamics simulation usually include the initial position and velocity of all atoms. This information is usually available from the experimental data or a theoretical model. Sometimes one combines both to obtain the initial conditions. In general, we start with a system having the initial density distribution $n(r)$ and the temperature distribution $T(r)$, which may not have any fixed periodicity. The initial position of each atom can be obtained from the screening method or the metropolis method, which leads to the initial density distribution. The initial velocity for each atom, however is obtained using the initial temperature to satisfy the Maxwell-Boltzmann random distribution. This Maxwell-Boltzmann distribution usually uses the distributed random number generator to obtain.

\subsection{Equilibrium ensemble system control method}

In the simulations of equilibrium NVT ensembles, NPT ensembles, or even NVE ensembles, it is often necessary to adjust the temperature to the desired value. For properties depending on the temperature, it is often needed to simulate the system at 
different temperatures. Another use of MD simulation is in search of the global minimum of the potential energy surface of a system. It is well known that the optimization schemes such as the steepest-descent method, conjugate-gradient method and NewtonRaphson method can easily fall into the metastable state. To overcome this difficulty, the simulated annealing method was proposed. The first step of this method is to increase the temperature to above the melting point of the system. Then the system is slowly cooled down to reach equilibrium at a very low temperature via MD simulations. Therefore, how to vary the temperature is a critical issue in MD simulations.

The temperature $T$ of the system is directly connected with the kinetic energy $K$. The relation can be written as:

$$
K=\sum_{i=1}^{N} \frac{1}{2} \cdot m_{i} \cdot\left|v_{i}\right|^{2}=\frac{\left(3 N-N_{c}\right)}{2} k_{B} T
$$

Here, $N$ is the number of atoms in the system, $N_{\mathrm{c}}$ is The number of constraints applied, $k_{\mathrm{B}}$ is the Boltzmann constant.

There are currently four methods used to adjust temperatures in MD calculations which are described below.

\subsubsection{Velocity scaling [27]}


The simplest way to incorporate the effect of temperature is to directly scale the velocity. Assume at time $t$ the temperature is $T(t)$. Now if the velocities of all the atoms are multiplied by a factor of $\lambda$, the temperature will change to:

$$
\begin{aligned}
& \Delta T=\left(\lambda^{2}-1\right) T(t) \\
& \lambda=\sqrt{\frac{T_{r e q}}{T(t)}}
\end{aligned}
$$

Here, $T_{\text {req }}$ is the targeted temperature at each step.

Thus, to control the temperature in each step is to multiply the velocities by the scale factor $\lambda$.

\subsubsection{Berenson thermal bath}

This approach is proposed by Berenson ${ }^{[28]}$ in 1984 . His model is that the system is in contact with a virtual hot bath whose temperature is maintained at a target value. Each step is scaled in such a way to reach the desired rate of temperature change of the heat bath which is proportional to the system temperature change $\left(T_{\text {bath }}-T(t)\right)$. For each step the temperature change is:

$$
\Delta T=\frac{\delta t}{\tau}\left(T_{b a t h}-T(t)\right)
$$

Therefore, the scaling factor of the velocity is: 


$$
\lambda=1+\frac{\delta t}{\tau}\left(\frac{T_{b a t h}}{T(t)}-1\right)
$$

When the coupling parameter is equal to the time step $\delta t$, this algorithm is equivalent to the simple velocity scaling method. The suitable value of $\tau$ is about 0.4 ps. Thereby, if the time step $t$ is $1 \mathrm{fs}$, we get $\delta t / \tau \approx 0.0025$. This method has the advantage that it allows the temperature of the system to fluctuate around the desired value.

\subsubsection{Gaussian thermal bath [29]}

The basic idea of this approach is to add a "friction" term in the equations of motion, where the force can be associated with the velocity of the system. The equation of motion is given by the following formula:

$$
\frac{m_{i} d^{2} r_{i}}{d t^{2}}=F_{i}-m_{i} \xi_{G} v_{i}
$$

Here, $\xi_{G}=\left(\sum_{j} v_{j} F_{j}\right)\left(\sum_{k} m_{k} v_{k} \cdot v_{k}\right)^{-1}$, which ensures the total kinetic energy be conserved. In this method, the initial temperature is usually set to the desired value. 


\subsubsection{Nose-Hoover thermal bath ${ }^{[30,31]}$}

This method uses the same equations of motion as the Gaussian method above, but the $\xi$ values are given differently.

$$
\frac{d \xi}{d t}=-\frac{1}{\tau_{N H}^{2}}\left(\frac{T}{T_{0}}-1\right)
$$

When $\tau_{N H} \rightarrow \infty, \xi=0$, the system is equivalent to an adiabatic system. When $\tau_{N H} \rightarrow \delta t, \xi=\xi_{G}$, the system is equivalent to what we get from the Gaussian method. How to select the value for $\tau_{N H}$ is system dependent. More discussion about this can be found in the references ${ }^{[32,33]}$.

\subsection{Periodic boundary conditions}

Periodic boundary conditions (PBCs) are often used in MD calculations to simulate macroscopic systems using a limited-size of cell. It removes the finite-size effect and the surface effect by constructing a quasi-infinite system. Mathematical representation of periodic boundary conditions is of the form:

$$
A(\vec{r})=A(\vec{r}+\vec{n} L) \vec{n}=\left(n_{1}, n_{2}, n_{3}\right)
$$

Here $A$ is an arbitrary observable; $n_{1}, n_{2}$ and $n_{3}$ are arbitrary integers. This boundary condition requires the supercell to repeat infinitely in a three-dimensional space. For example, when a particle passes through the boundary of a supercell in a MD simulation, 
the velocity of the particle is set to be the same across the boundary when it re-enters the neighboring cell. This process is illustrated in Figure 6.3.

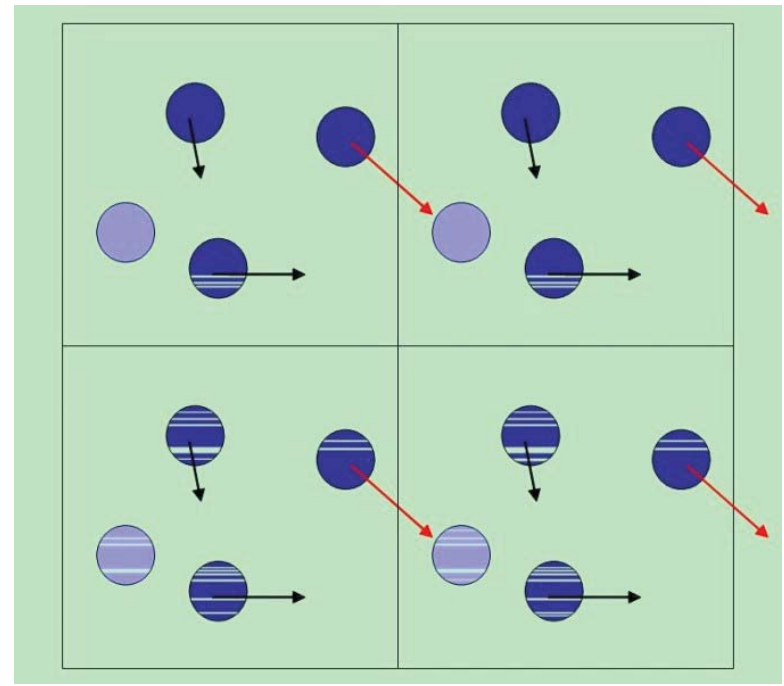

Figure 6.3 Illustration of the periodic boundary conditions for a particle passes through the boundary of a supercell in a MD simulation.

\subsection{Basic steps}

Molecular dynamics simulations can be conducted through four major steps:

\subsubsection{Build a simulation model}

The very first step is to build a simulation model, and choose or construct the proper force fields. This can be done either through literature search or through original development of force fields using first-principles results and experimental data. Depending upon the type of interaction in the physical system, different potential 
functions may be chosen. And often it is the most critical step to ensure getting reliable data from the simulation.

\subsubsection{Set initial conditions}

The initial positions are given based on the structural information of the system, and the initial velocities are obtained by random sampling from the Boltzmann distribution.

\subsubsection{Equilibrate the system}

Once the boundary and initial conditions are set, MD simulations can be carried out following the equations of motion. Be aware that such a system is not in an equilibrium state yet. It may take quite some time for the system to reach equilibrium. In this process, the total free energy of the system may either increase or decrease until it reaches a state that the total energy only fluctuate around a certain value. The time required to reach equilibrium is called the relaxation time.

\subsubsection{Determination of macroscopic quantities}

Many of the physical quantities of a given system require knowledge of the statistical physics for their determination by a MD simulation.

\subsubsection{Radial distribution function}

If $n$ is the average number of particles between the distance $r$ to $r+\Delta r$, the radial distribution function of the system can be obtained from this expression: 


$$
g(r)=\frac{V}{N} \frac{n(r)}{4 \pi r^{2} \Delta r}
$$

.The radial distribution function usually refers to a given coordinate of a particle $i$. The probability distribution of all the other particles in the $i$-space can be expressed as

$$
g_{i, j}(r)=\frac{V}{N_{i} N_{j}} \sum_{i}^{N_{i}} \frac{n_{i, j}\left(r-\frac{\Delta r}{2}, r+\frac{\Delta r}{2}\right)}{4 \pi r^{2} \Delta r}
$$

Where, $N_{\mathrm{i}}$ and $N_{\mathrm{j}}$ are the total number of atoms of type $i$ and $j$ in the system, $n_{\mathrm{i}, \mathrm{j}}$ represents the number of $j$ atoms within the distance of $r-\Delta r / 2$ to $r+\Delta r / 2$ from the center $i$ atom. If $i, j$ represents the same type of atom, then $N_{\mathrm{j}}=N_{\mathrm{i}-1}$. The radial distribution function can be used to study the orderliness of material and also to describe the electron correlation.

\subsubsection{Kinetic energy}

During the simulation, the kinetic energy of particles does not follow a continuous path. Assume each discontinuous for $\mu$ points of time, the average kinetic energy can be calculated as:

$$
\overline{E_{k}}=\frac{1}{n-n_{0}} \sum_{\mu>n_{0}}^{n} \sum_{i=1}^{N} \frac{\left(P_{i}^{2}\right)^{(\mu)}}{2 m}
$$

On the other hand, the temperature needs to be monitored anytime during a MD simulation, in particular the initial stage of the simulation. According to the energy 
equipartition theorem, we can calculate the value of the average kinetic energy from the temperature value; the calculation can be expressed as:

$$
T=\frac{\overline{E_{k}}}{\frac{d}{2} N k_{B}}
$$

Here $d$ is the degree of freedom for each particle. If there is no constraints for the system, then $d=3$.

\subsubsection{Potential energy}

The average Potential energy of the system is calculated by:

$$
\bar{U}=\frac{1}{n-n_{0}} \sum_{\mu>n_{0} i>j}^{n} \sum_{i}^{n} u\left(r_{i, j}^{(\mu)}\right)
$$

If the local potential energy is truncated at $r_{\mathrm{c}}$, this formula may lead to errors in the calculated potential energy. In order to avoid this, we use the radial distribution function $g(r)$ in the expression of the local potential energy and the average potential energy of the system can be calculated as follows:

$$
\frac{U}{N}=2 \pi p \int_{0}^{\infty} U(r) g(r) r^{2} d r
$$




\section{References}

[1] J. Schiøtz, F. D. Di Tolla, and K. W. Jacobsen: "Softening of nanocrystalline metals at very small grain sizes", Nature 391, (1998) 561-563.

[2] A. Hasnaoui, H. Van Swygenhoven, and P. M. Derlet, "On non-equilibrium grain boundaries and their effect on thermal and mechanical behaviour: a molecular dynamics computer simulation". Acta Materialia, Volume 50, Issue 15, 3 September (2002) 39273939.

[3] van Gunsteren, Wilfred F., and Herman JC Berendsen. "Computer simulation of molecular dynamics: Methodology, applications, and perspectives in chemistry." Angewandte Chemie International Edition in English 29, no. 9 (1990) 992-1023.

[4] Pearlman, David A., David A. Case, James W. Caldwell, Wilson S. Ross, Thomas E. Cheatham III, Steve DeBolt, David Ferguson, George Seibel, and Peter Kollman. "AMBER, a package of computer programs for applying molecular mechanics, normal mode analysis, molecular dynamics and free energy calculations to simulate the structural and energetic properties of molecules." Computer Physics Communications 91, no. 1 (1995) 1-41.

[5] Stillinger, Frank H., and Thomas A. Weber. "Computer simulation of local order in condensed phases of silicon." Physical Review B, 31, no. 8 (1985) 5262. 
[6] GUNSTEREN, Wilfred F., and Alan E. Mark. "On the interpretation of biochemical data by molecular dynamics computer simulation." European Journal of Biochemistry, 204, no. 3 1992: 947-961.

[7] Lennard-Jones, J. E. "On the Determination of Molecular Fields", Proc. R. Soc. Lond. A 106 (738), (1924) 463-477.

[8] A. Vedani.Yeti. "An interactive molecular mechanics program for small-molecular protein complexes". Journal of Computational Chemistry, 9: 269, (1988).

[9] Brooks, Bernard R., et al. "CHARMM: A program for macromolecular energy, minimization, and dynamics calculations." Journal of computational chemistry 4.2 (1983) 187-217.

[10] Allinger, Norman L., Young H. Yuh, and Jenn Huei Lii. "Molecular mechanics. The MM3 force field for hydrocarbons. 1." Journal of the American Chemical Society $111.23(1989) 8551-8566$.

[11] Lii, Jenn Huei, and Norman L. Allinger. "Molecular mechanics. The MM3 force field for hydrocarbons. 2. Vibrational frequencies and thermodynamics." Journal of the American Chemical Society 111.23 (1989) 8566-8575.

[12] Lii, Jenn Huei, and Norman L. Allinger. "Molecular mechanics. The MM3 force field for hydrocarbons. 3. The van der Waals' potentials and crystal data for aliphatic and 
aromatic hydrocarbons." Journal of the American Chemical Society 111.23 (1989) 85768582.

[13] Allinger, Norman L., Kuohsiang Chen, and Jenn - Huei Lii. "An improved force field (MM4) for saturated hydrocarbons." Journal of Computational Chemistry 17.5 - 6 (1996) 642-668.

[14] Nevins, Neysa, Kuohsiang Chen, and Norman L. Allinger. "Molecular mechanics (MM4) calculations on alkenes." Journal of computational chemistry 17.5 - 6 (1996) 669-694.

[15] N. Nevins, J.-H. Lii, and N. L. Allinger. "Molecular mechanics (MM4) calculations on conjugated hydrocarbons". J. Comput. Chem 17 (1996) 695-729.

[16] S. J. Weiner, P. A. Kollman, D. A. Case, U. C. Singh, C. Ghio, G. Alagona, S. Profeta, and P. Weiner. "A new forcefield for molecular mechanical simulation of nucleic acids and proteins". J. Am. Chem. Soc 106, (1984) 765-784.

[17] W. D. Cornell, P. Cieplak, C. I. Bayly, I. R. Gould, K. M. Merz, D. M. Ferguson, D. C.Spellmeyer, T. Fox, J. W. Caldwell, and P. A. Kollman. "A 2nd generation forcefield for the simulation of proteins, nucleic-acids, and organic molecules". J. Am. Chem. Soc 117, (1995) 5179-5197. 
[18] W. L. Jorgensen, D. S. Maxwell, and J. TiradoRives. "Development and testing of the OPLS all-atom forcefield on conformational energetics and properties of organic liquids". J. Am. Chem. Soc 118, (1996) 11225-11236.

[19] Izvekov, Sergei, and Gregory A. Voth. "A multiscale coarse-graining method for biomolecular systems." The Journal of Physical Chemistry B 109.7 (2005) 2469-2473.

[20] Izvekov, Sergei, and Gregory A. Voth. "Multiscale coarse graining of liquid-state systems." The Journal of chemical physics 123.13 (2005) 134105.

[21] Müller-Plathe, Florian. "Coarse-Graining in Polymer Simulation: From the Atomistic to the Mesoscopic Scale and Back." ChemPhysChem 3.9 (2002) 754-769.

[22] Verlet, Loup. "Computer "Experiments" on Classical Fluids. I. Thermodynamical Properties of Lennard-Jones Molecules". Physical Review 159, (1967) 98-103.

[23] Swope, William C., et al. "A computer simulation method for the calculation of equilibrium constants for the formation of physical clusters of molecules: Application to small water clusters." The Journal of Chemical Physics 76.1 (1982) 637-649.

[24] R. W. Hockney and J. W. Eastwood. "Computer simulations using particles". Adam Hilger, Bristol, 1988.

[25] CW Gear. "The simultaneous numerical solution of differential-algebraic equations". IEEE Trans. Circuit Theory TC, 18 (1) (1971) 89-95. 
[26] Beeman, D. "Some multistep methods for use in molecular dynamics calculations." Journal of Computational Physics 20, no. 2 (1976) 130-139.

[27] H. C. Andersen. "Molecular dynamics simulations at constant pressure and/or temperature". J. Chem. Phys. 72 (1980) 2384-2393.

[28] Berendsen, Herman JC, et al. "Molecular dynamics with coupling to an external bath." The Journal of chemical physics 81.8 (1984): 3684-3690.

[29] Hoover, William Graham. "Computational statistical mechanics.” Access Online via Elsevier, 1991.

[30] Nosé, Shūichi. "A molecular dynamics method for simulations in the canonical ensemble." Molecular physics 52.2 (1984) 255-268.

[31] Hoover, William G. "Canonical dynamics: Equilibrium phase-space distributions."Physical Review A 31.3 1985, 1695.

[32] Hoover, William G. "Canonical dynamics: equilibrium phase-space distributions." Physical Review A 31.3 (1985): 1695-1697.

[33] Andersen, Hans C. "Molecular dynamics simulations at constant pressure and/or temperature." The Journal of chemical physics 72.4 (2008) 2384-2393. 


\section{Chapter 7}

\section{Interactions of Metallic Nanoparticles with Lipid}

\section{Bilayers}

\subsection{Introduction}

Metallic nanoparticles such as silver and gold nanoparticles are among the most researched nanomaterials for the past decade. At the size of 1-100 nm, these nanoparticles exhibit fascinating electrical, magnetic, and optical properties. ${ }^{[1,2]}$ Silver and gold nanoparticles have found many important applications in industry and commercial use. For example, silver nanoparticles (AgNPs) have been widely used as high-efficiency inorganic antibacterial materials ${ }^{[3]}$ in medicine, biology, environmental protection and other fields. In addition, AgNPs have shown good antimicrobial bactericidal effect in burn surgery, dermatology, dentistry and other clinical care ${ }^{[4-6]}$. Gold nanoparticles (AuNPs) are also widely used nanomaterial with several advantages over other metallic nanoparticles. They can be easily synthesized. They have stable chemical properties and have the same surface tunable properties as observed for AgNPs. AuNPs also have numerous applications in biological systems. 
Recently, AuNPs has been used as drug delivery agents ${ }^{[7,8]}$, DNA transfection vectors ${ }^{[9]}$, diagnostic markers ${ }^{[10]}$ and protein inhibitors ${ }^{[11]}$.

In drug-delivery applications, AgNPs and AuNPs are required to enter biological cells in order to reach the targeted cellular compartments. Thus, during this process, AgNPs and AuNPs have to transverse the cell membrane which includes a hydrophobic barrier. It has been demonstrated that the interaction of AgNPs and AuNPs with cell membranes tremendously influences their cellular uptake as well as their cytotoxicity ${ }^{[12-15]}$. Therefore, a comprehensive investigation of the interaction of cell membranes with AgNPs and AuNPs at the atomic level is a crucial task in better understanding the effects of metallic nanoparticles in biological applications.

Experimental results show that the AgNPs are able to cause damage on bilayer membranes. Leroueil et al. demonstrated that cationic AgNPs can generate defects on supported lipid bilayers (SLB) ${ }^{[16]}$. Other experiments also show that the AgNPs not only can kill the test cell but also can change the cell's shape and make the cell to look very ugly ${ }^{[17]}$. On the other hand, cationic AuNPs are able to release fluorescent dyes from vesicles by interrupting the lipid bilayer ${ }^{[18]}$. These experiments have given the evidence of toxicity of metallic nanoparticles towards biological cells. However, very little is known regarding how this happens due to existence of multiple components and the complexity of the interaction. Considering that the nanoparticles first encounter the cell membrane on their way into the cell, the study of the nanoparticles interacting with the membrane is an important topic for an 
understanding of the cytotoxicity. However, the details of this interaction at molecular level is largely lacking and beyond the capability of the current experimental technologies. MD simulations, on the other hand, provide an effective alternative for such investigations; they can facilitate information at "impossible angles" which are inaccessible for experiments.

The simulations of nanoparticles interacting with biological systems are often challenging because of the complexity of a nano-bio system. There have been only a few simulation studies engaged in these issues. Previously studies were reported on the interactions of fullerenes ${ }^{[19-21]}$, carbon nanotubes ${ }^{[22]}$, and dendrimers with bilayer membranes ${ }^{[23]}$. The interaction of metallic nanoparticles with membranes was considered by a few scientists. Liu et al. studied the effect of AuNPs surface charge on the membranes ${ }^{[24]}$, and the formation of holes on bilayer membranes caused by AuNPs ${ }^{[25]}$. In their work, only the electrostatic Coulomb forces due to the charged species were taken into account for the interaction between the metal nanoparticles and the membranes. There is a certain limitation to this model which will be address in this chapter.

A new force field model was developed to describe the interaction between the metal cluster and the lipid bilayer. This force field together with the force field describing the biological system itself was then employed to conduct a full study of the AuNPs interacting with a lipid bilayer. 


\subsection{Simulation model}

The relatively low computational cost of a MD calculation compared to the firstprinciple calculation makes it a powerful tool in simulating a large system even of over one million atoms, which appears to be critical sometimes for getting a realistic description of a biological system. It also becomes challenging to build such a huge simulation system. It is the very first step one needs to take in a MD simulation.

Considering that membranes are mainly composed of lipid, we choose to use the double-layer lipid as a simplified model for the membrane. First of all, we build the support lipid membranes (SLB) through an online tool (the website: http://www.charmmgui.org/?doc=input/membrane). Using the membrane builder a SLB system is constructed consisting of a hydrophilic support with a DMPC bilayer $(2 \times 64$ DMPC molecules $)$ and 10882 TIP3 water molecules. For each bilayer, a cubic simulation box is used in which the bilayer is submerged in water. In order to simulate the nanoparticles interacting with the membrane, which is a two-dimensional infinite structure, we use the periodic boundary conditions (PBCs). The initial box size was set to $6.23 \times 6.23 \times 12.00 \mathrm{~nm}^{3}$. We also add the salt effect in this system. The concentration of salt ions was set to $0.01 \mathrm{~mol} / \mathrm{L}$ by adding 29 salt ions in the aqueous solution.

We have chosen $\mathrm{Au}_{13}$ NPs and $\mathrm{Ag}_{13}$ NPs as our models for $\mathrm{Au}$ and $\mathrm{Ag}$ nanoparticles. These clusters have an approximately spherical shape with a diameter of $1 \mathrm{~nm}$. These clusters were pre-optimized using the DFT method. To reduce the simulation time, the 
NP was initially put very close to the surface of the SLB, and then, the system was relaxed (with the center-of-mass of the NP and lipid bilayer constrained) until the whole system reached its equilibrium state. The simulated system is shown in Figure 7.1.

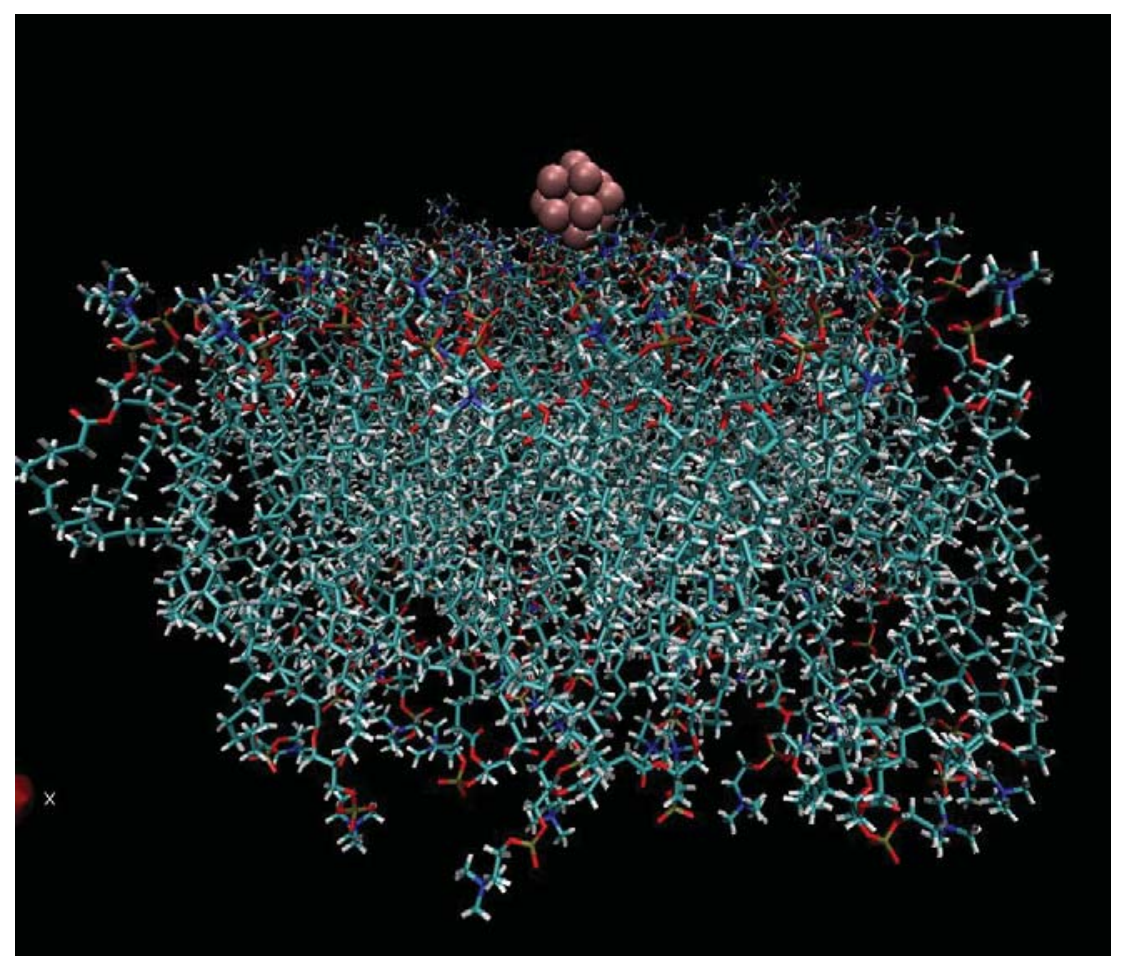

Figure 7.1: The initial simulation system of AuNPs-SLB in water. (For clarity, water molecules are not shown. The snapshot is rendered by VMD). 


\subsection{Force field}

The commonly-used Charmm-36 molecular force field was selected to simulate the membrane system. As demonstrated before, if the focus is on the interactions among biological systems (major components $\mathrm{C}, \mathrm{H}, \mathrm{O}$ ) such as protein interacting with membranes or biological systems interacting with carbon-based nanomaterials such as carbon nanotubes $(\mathrm{CNT}){ }^{[22]}$, the conventional Charmm-36 molecular force field is probably Satisfied the requirements, since it has already included van der Waals forcesthe leading interaction forces among them. In these cases, the need to modify the molecular force field or construct a new force field is minimal. However, the interaction of metal nanoparticles with organic compound is very different. Due to the high difference in the electronegativity of the metal and the constituting elements of biological molecules, the lipid in this case, the interaction between the metal nanoparticles and the biological system is featured by much stronger interaction than the van der Waals force. This has to be taken into account when we simulate the interaction of metal nanoparticles with membranes.

In these simulations, we have carefully chosen a near-spherical metal cluster; thereby we can ignore any effect from the shape of nanoparticles. But still the complexity comes from the DMPC molecule, which has several chemically active functional groups. Therefore, if the nanoparticle comes in from a different direction and interacts with DMPC at different site, the bonding energy could be largely different. In order to take into account all possibilities, all the active functional groups of the DMPC molecule are 
considered, namely amido, phosphine and fatty acid groups. These three sites are marked as NTL-site, CL-site and PL-site in Figure 7.2. The interaction of metal nanoparticles with these functional groups were conducted using the cluster model (Figure 7.2), where all the dangling bonds at the cleavage points are saturated by hydrogen atoms.

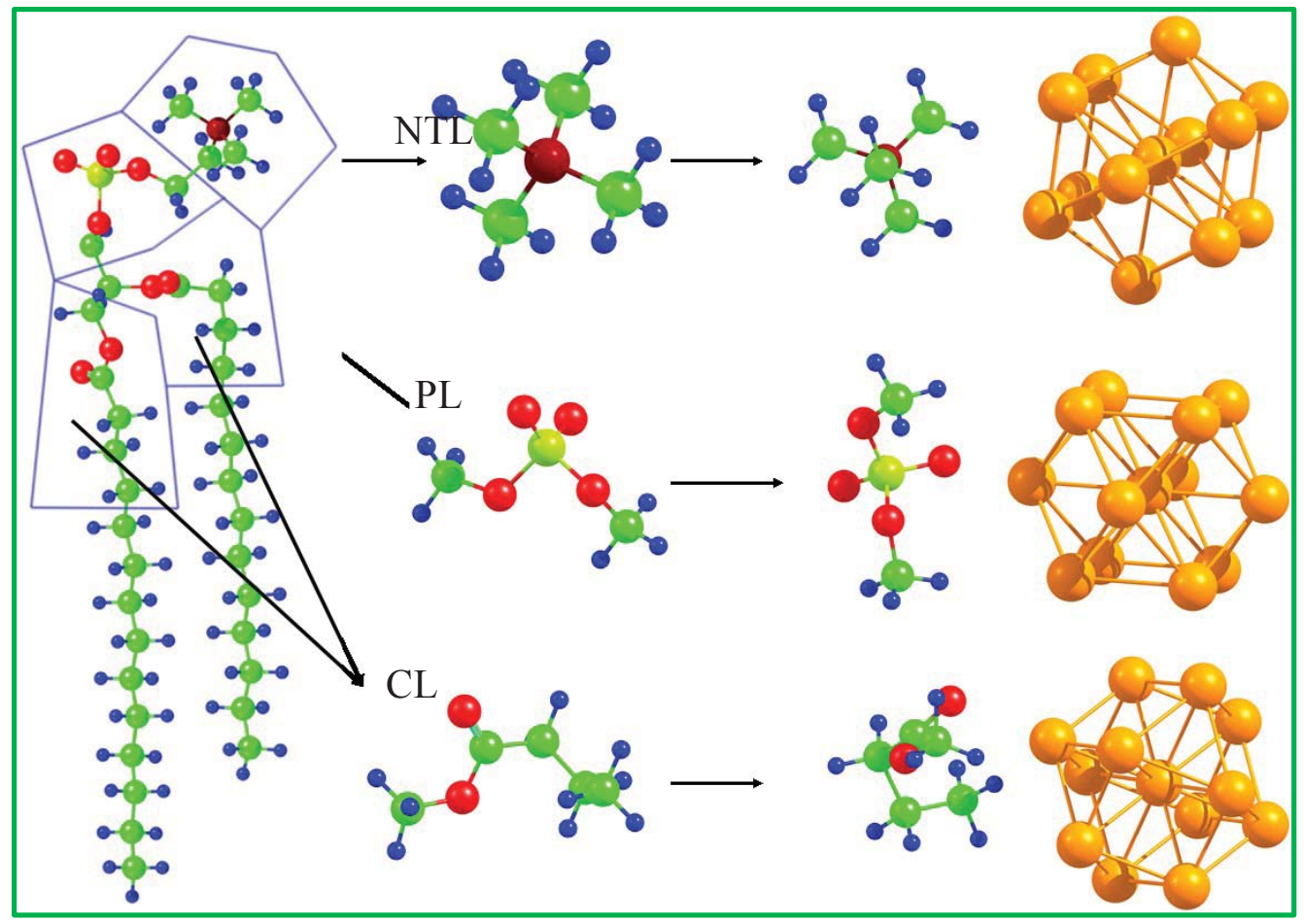

Figure 7.2: Illustration of the active chemical functional groups of a DMPC molecule and the cluster model considered for nanoparticles.

Simulation of interacting nanoparticles with membranes also brings the question of the stability of nanomaterials in a chemical environment. In general, nanoparticles are produced under ultrahigh vacuum conditions, and will eventually be exposed to air and/or 
water (at least) when they interact with cells. Therefore, the solvent effect could be significant and should be included in developing new force fields.

We start with quantum mechanical calculations to get an accurate account of the interaction using the cluster model. The Gaussian software was chosen to calculate the interaction. The solvent effect was included via the polarizable continuum model (PCM) model. The B3LYP hybrid exchange-correlation functional form and the LanL2MB basis sets were chosen. Partial optimization was conducted, where the AuNPs were fixed in position and only the functional groups were allowed to relax. All the results are presented in Figure 7.3.

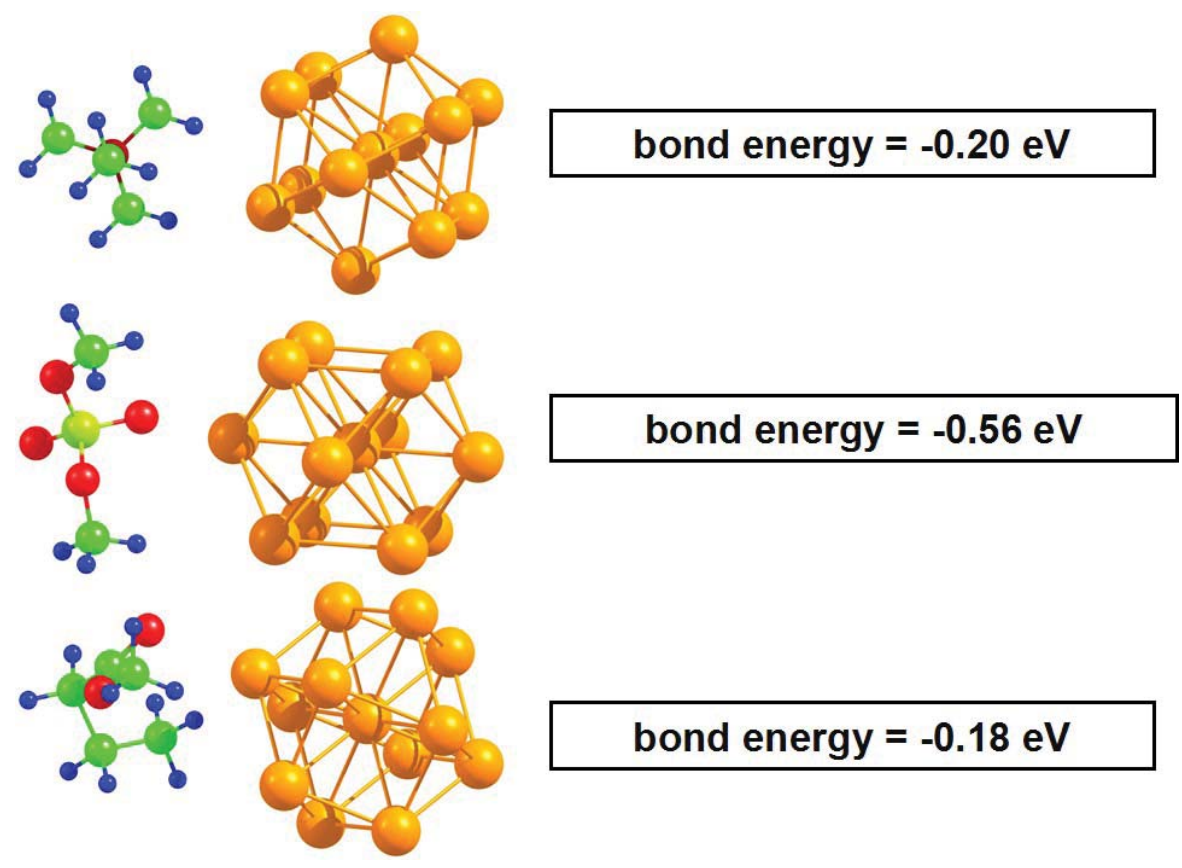

Figure 7.3: $\mathrm{Au}_{13}$ Cluster interacting with active sites of a DMPC molecule. 
The next step is to use these results to build a new force field. Our focus is to reproduce the interaction between the metal nanoparticles and the lipid using the force fields. In general, there are two ways to consider the interaction. One model is to describe it as the intermolecular and the other model is to count it as the intramolecular interaction. Previous studies mostly treat the interaction using the intermolecular model. For instance, van der Waals forces are adopted to reflect the interaction of carbon nanotubes or fullerenes with biological molecules. In some studies, some charged organic compounds are coated on the surface of metal nanoparticles, and they naturally lead to use of a form representing the electrostatic interaction. Considering the fact that the interaction of metal nanoparticles with the lipid biomolecules has a relatively strong chemical bonding feature, the use of the intermolecular interaction model is no longer sufficient. Therefore, we propose to treat metal nanoparticles and biomolecules as a whole and describe the interaction between them using the intramolecular interaction model. Recall that there are generally two-body, three-body and four-body terms to represent the intramolecular interactions. But since we are taking the relatively small metal cluster as a whole and it has a nearly spherical shape, the threebody (involving bond angles) and four-body (involving dihedral angles) terms are ignored and only the two-body term is included. This two-body interaction is described by the Lennard-Jones equation. Namely:

$$
U=E_{\text {bond }} \cdot\left[\left(\frac{R}{r}\right)^{12}-2 \cdot\left(\frac{R}{r}\right)^{6}\right]
$$


Here, $E_{b o n d}$ is the bond strength and $R$ is the equilibrium distance from the AuNPs to the lipid surface. More specifically, we take $R$ to be distance from the center of mass of the nanoparticle (i.e. Au7 atom) to the core reference atoms of the lipid functional groups (i.e. $\mathrm{N}$ of the amido group, the middle $\mathrm{C}$ atom of the fat acid and $\mathrm{P}$ in phosphine group). These two-body interactions now can be added to the Charmm-36 force field whose parameters are obtained by fitting into the DFT results (Table 7.1). We use the same method for the AgNPs and the results are listed in Table 77.2.

Table 7.1: Force field parameters describing the interaction of the AuNPs with DMPC.

\begin{tabular}{|c|c|c|c|}
\hline $\mathbf{A u N P}$ & $\begin{array}{c}\text { DMPC } \\
\text { functional } \\
\text { group }\end{array}$ & $\begin{array}{c}\boldsymbol{E}_{\text {bond }} \\
\mathbf{( k c a l} / \mathbf{m o l})\end{array}$ & $\boldsymbol{R}(\boldsymbol{\AA})$ \\
\hline $\mathrm{Au}_{7}$ & $\mathrm{NTL}$ & 4.836 & 6.764 \\
\hline $\mathrm{Au}_{7}$ & $\mathrm{PL}$ & 12.896 & 6.210 \\
\hline $\mathrm{Au}_{7}$ & $\mathrm{CL}$ & 4.606 & 6,953 \\
\hline
\end{tabular}

Table 7.2: Force field parameters describing the interaction of the AgNPs with DMPC.

\begin{tabular}{|c|c|c|c|}
\hline$A g N P$ & $\begin{array}{c}\text { DMPC } \\
\text { functional } \\
\text { group }\end{array}$ & $\begin{array}{c}\boldsymbol{E}_{\text {bond }} \\
(\mathbf{k c a l} / \mathbf{m o l})\end{array}$ & $\boldsymbol{R}(\AA)$ \\
\hline $\mathrm{Ag}_{7}$ & $\mathrm{NTL}$ & 4.751 & 6.538 \\
\hline $\mathrm{Ag}_{7}$ & $\mathrm{PL}$ & 10.526 & 6.317 \\
\hline $\mathrm{Ag}_{7}$ & $\mathrm{CL}$ & 5.757 & 6.412 \\
\hline
\end{tabular}




\subsection{MD simulation}

With the newly developed molecular force field, now we are ready to carry out MD simulations for the system described in Section 7.2. Initially, the nanoparticle was placed $2 \AA$ above the lipid bilayer solvated in the bulk water solution. Periodic boundary conditions were applied. The time step of integration is $1 \mathrm{fs}$. This effective time step is used throughout this study. Temperature was set to be $303.05 \mathrm{~K}$ and we choose Langevin dynamics to control the constant temperature and coupling to the system in a NPT ensemble. The target pressure is 1 bar and the Nose-Hoover model is used to control pressure. Ewald method was applied to address the long-range electrostatic interactions. Short-ranged electrostatic and van de Waals interactions have a cutoff radius

of $3.0 \mathrm{~nm}$. All simulations were performed by the NAMD 2.9 package. ${ }^{[26]}$ For the first $0.5 \mathrm{~ns}$, the system was under planar and dihedral restraints. Then the planar restraint and the dihedral restraint were removed. The system was run for about $10 \mathrm{~ns}$ and this was then used for analysis. The time to reach equilibrium varies from $4.5 \mathrm{~ns}$ for AuNPs to $0.25 \mathrm{~ns}$ for AgNPs.

\subsection{Results and discussion}

\subsubsection{Permeation of AuNPs into Lipid Bilayers}

The process of insertion of the AuNPs into the bilayer membrane is shown in Figure 7.4. AuNPs was initially placed $0.2 \mathrm{~nm}$ above the lipid bilayer surface using the Nitrogen atoms as reference. From these trajectories, we can clearly see that once the simulation 
started, the AuNPs began to move down toward the bilayer very quickly. When they were in contact, the AuNPs soon penetrated into the bilayer interior. It is also observed that the headgroups of the lipid bilayer stay close to the AuNPs as if being coated on the surface of AuNPs. Once the AuNPs penetrated into the bilayer interior, it interacts with the lipid phosphodiester groups. Note this is different in the case of fullerene or carbon nanotubes, which can rapidly cross the region of the lipid headgroups and diffuse more slowly in the membrane interior. The process of insertion of $\mathrm{Au}_{13}$ particle was spontaneous and happened in less than 1ps. The insertion process also depends on the initial distance between the NPs to the membrane surface. Some of lipid phosphodiester groups coated AuNPs forming a "hump" in order to accommodate the invasion of the AuNPs. Because the three chemical functional groups have the strong interaction with AuNPs, the lipid phosphodiester groups surrounding the nanoparticle were disordered and hydrated forming a nanoparticle-lipid complex. On the other hand, since the thickness of the lipid bilayer is about $5.0 \mathrm{~nm}$ (this value depends on the fat acid length), the metal nanoparticles have very weak interaction with the alkyl group. These two factors make the AuNPs hardly affect the other side of the lipid bilayer.

Our results show that the effect of the charge-neutral AuNPs on the lipid membrane is very different from that of the positively-charged cationic AuNPs, where the charge comes from the organic compound coating on the AuNPs surface. In the latter, the cationic AuNPs interaction with the lipid bilayer is dominated by the electrostatic Coulomb interaction owing to opposite charges ${ }^{[24]}$. In our case, however, the AuNPs 
interaction with the lipid bilayer is mostly governed by the interaction of the metal nanoparticle with three active chemical functional groups of the lipid. Differences in the active groups determine the difference in their effect on the lipid membrane. The carbonbased fullerenes and carbon nanotubes are not like the metallic nanomaterial, which interacts more strongly with the lipid hydrophilic heads. Carbon-based nanomaterials can rapidly transverse the region of the lipid headgroups staying at the region of the hydrophobic tail, owing to their relatively weak interactions with membranes. For the AgNPs we also get the same results.
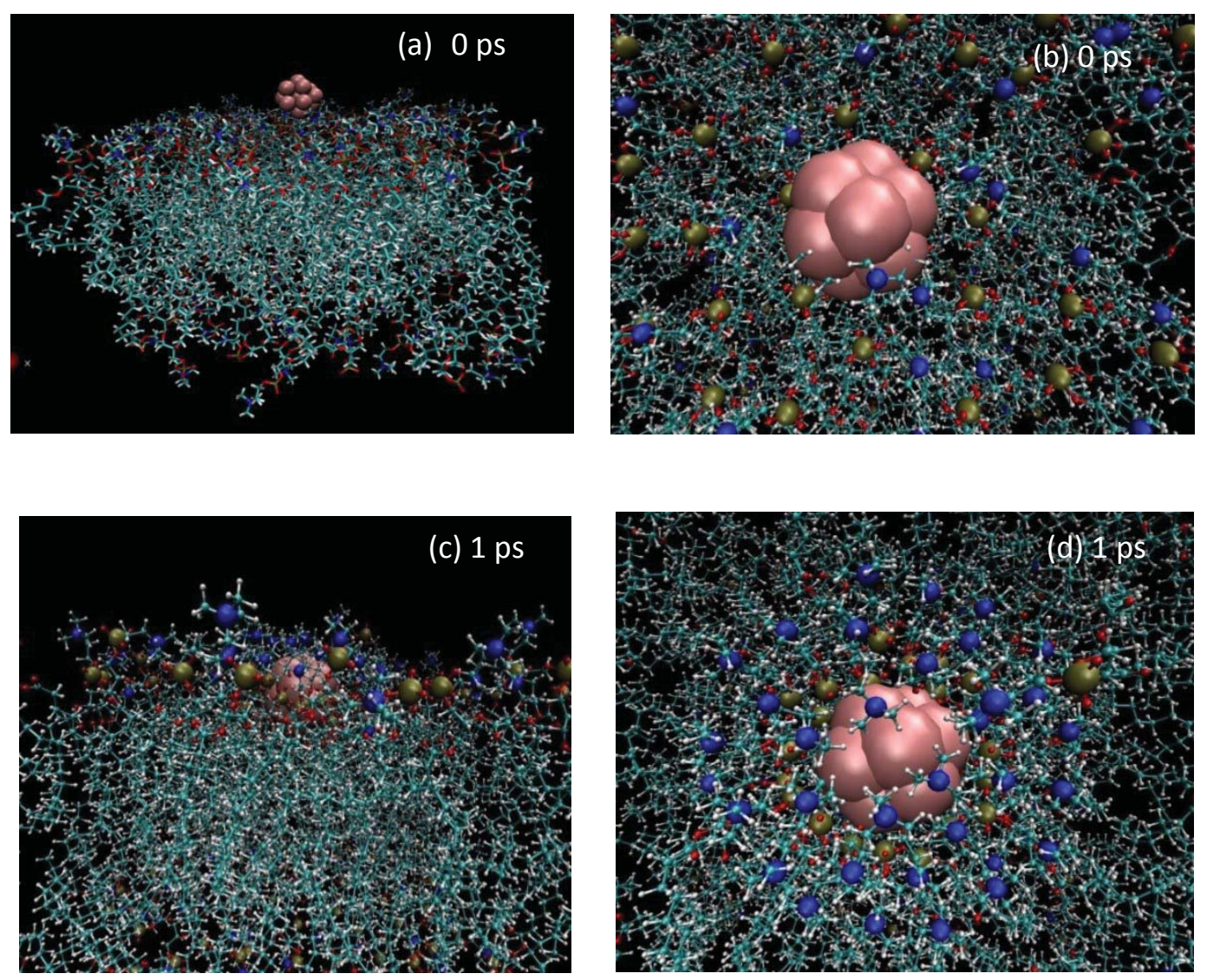

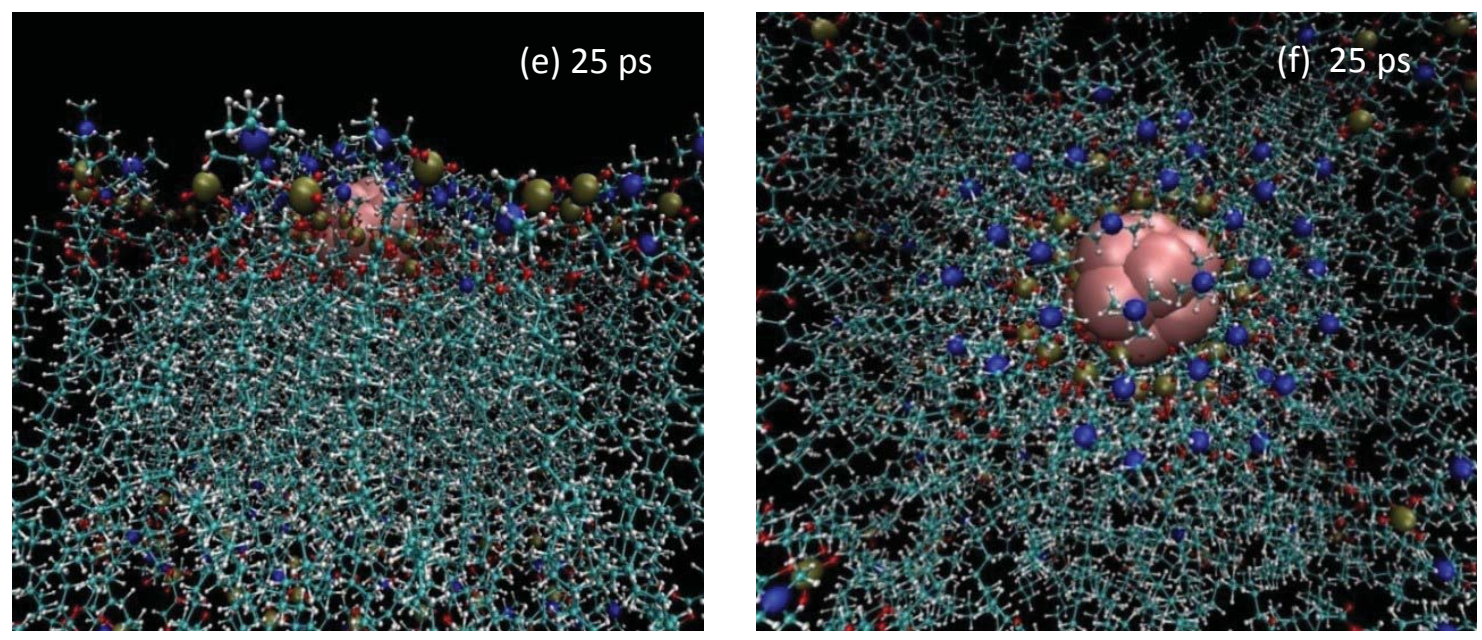

Figure 7.4: Simulated permeation process of AuNPs through a lipid membrane: (a) side and (b) top views of the initial position of a AuNP near the surface of a lipid bilayer; (c) side and (d) top views of the position of a AuNP after 1 ps; (e) side (f) top views of the position of a AuNP after 25 ps.

\subsubsection{Analysis}

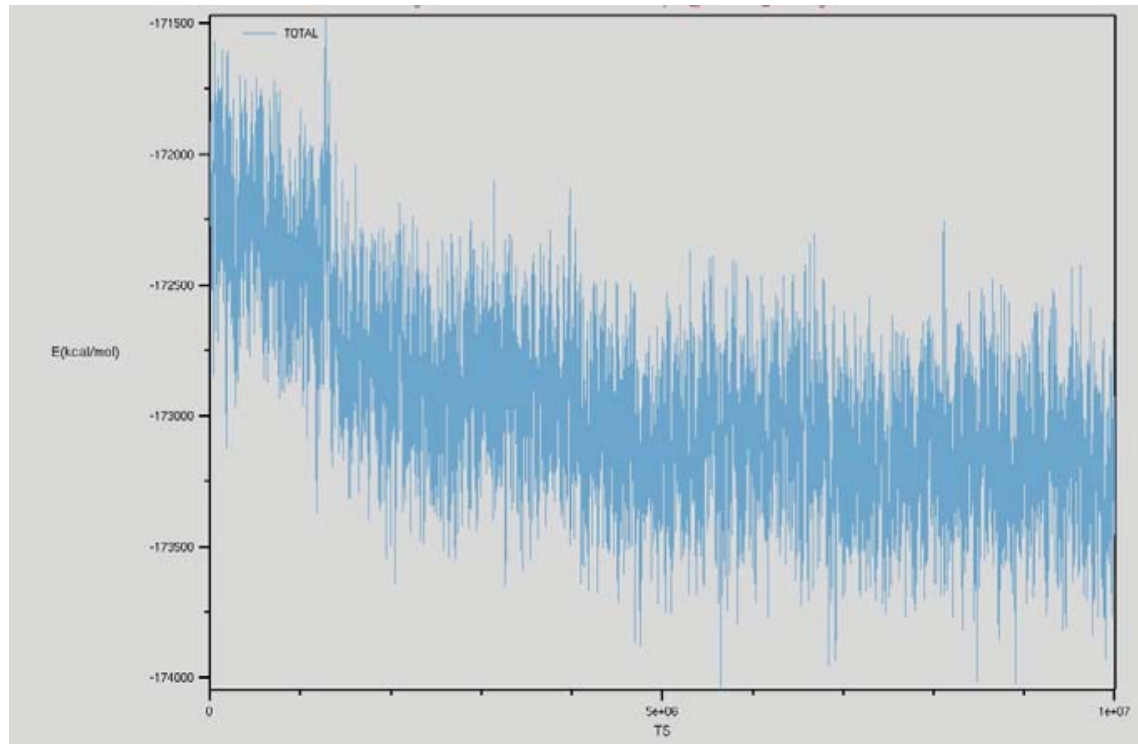

Figure 7.5: Total energy variation as a function of the simulation time for AuNPs. 


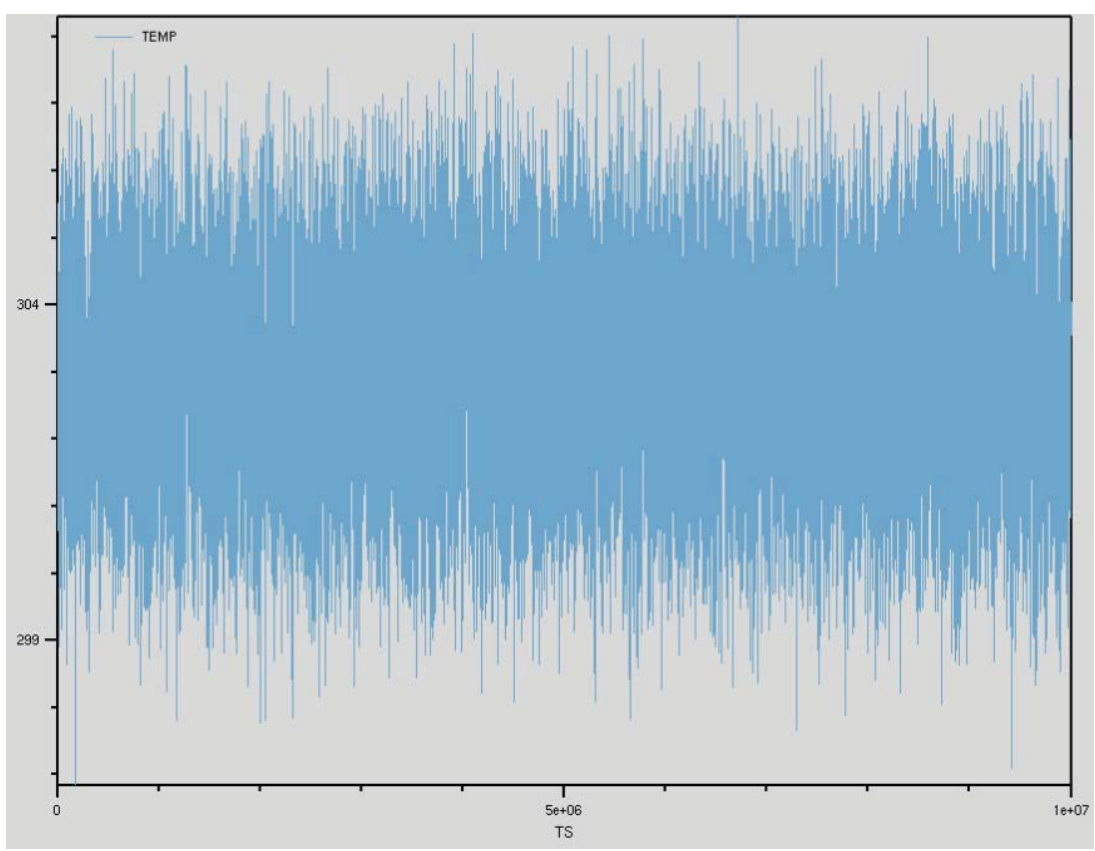

Figure 7.6: Temperature fluctuation as a function of the simulation time for AuNPs.

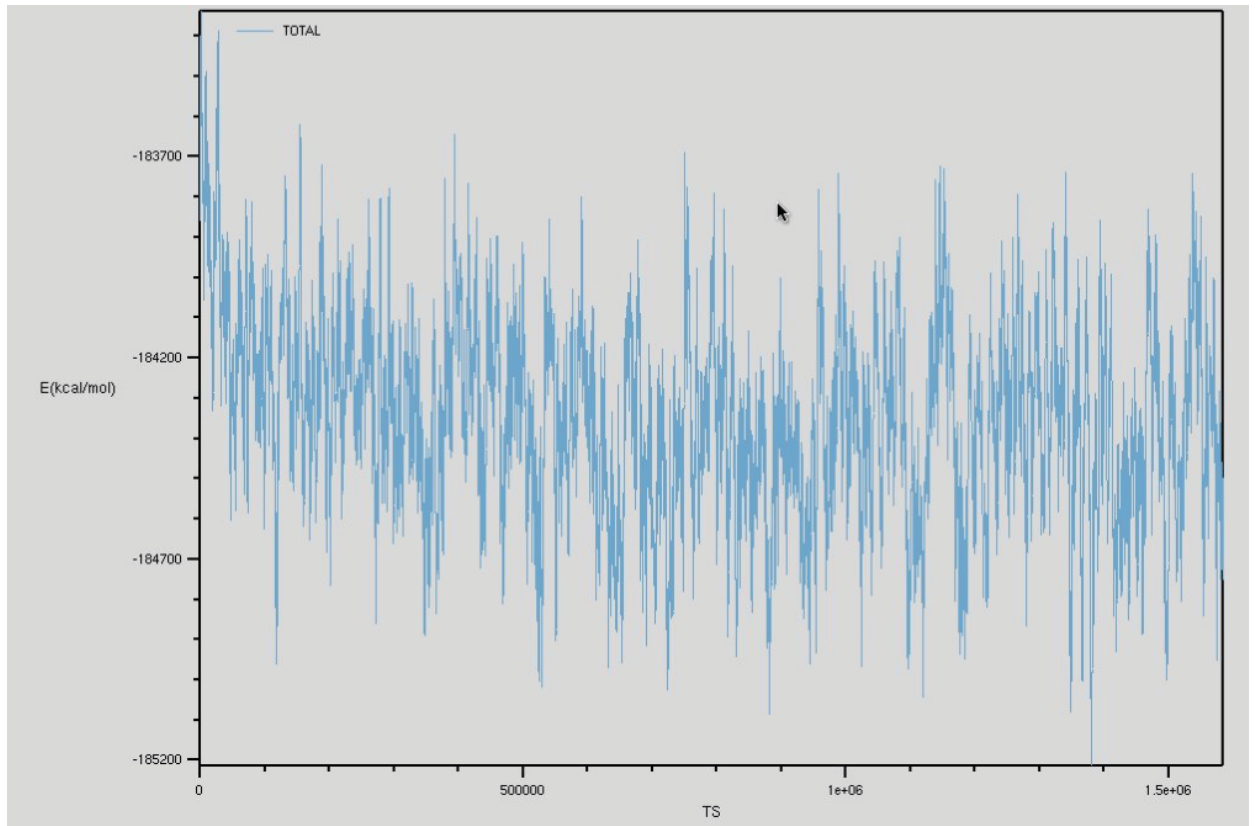

Figure 7.7: Total energy variation as a function of the simulation time for AgNPs. 


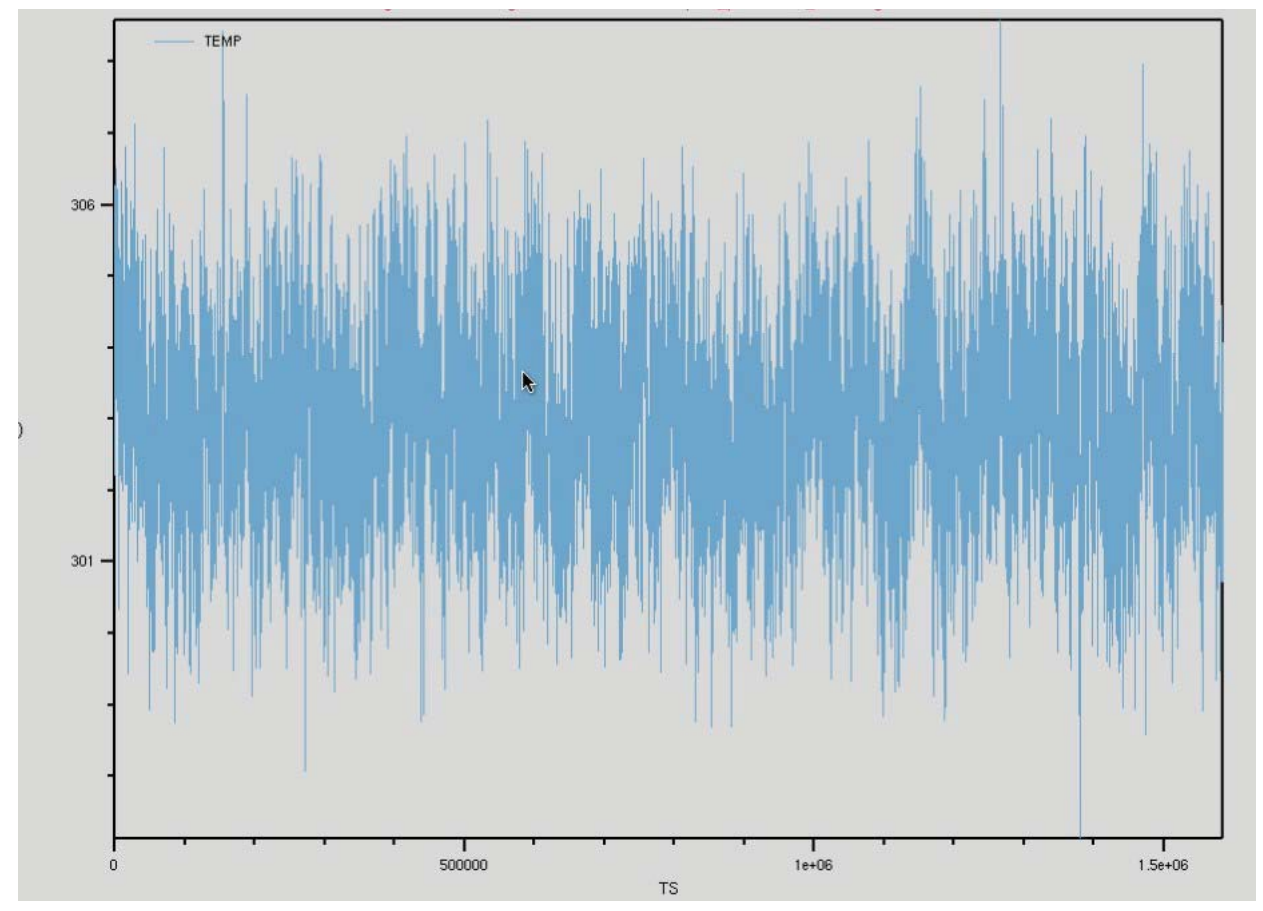

Figure 7.8 Temperature fluctuations as a function of the simulation time for AgNPs.

Some physical quantities such as the total energy and the temperature are plotted as a function of the simulation time in Figures 7.5, 7.6 and Figures 7.7, 7.8 for the interaction with AuNPs and AgNPs, respectively. The total energy of the AuNP/AgNP-lipid system is analyzed as an indicator of reaching the equilibrium state. It is also used to study the thermodynamics of the system in the presence of the AuNPs or AgNPs.

From fluctuation of the total energy, we can tell that the AuNP-lipid system arrives at the equilibrium state after $4.5 \mathrm{~ns}$ and the energy fluctuates around a certain value. The AgNPs-lipid system can arrive at the equilibrium state more rapidly than the AuNP-lipid system. Figures 7.6 and 7.8 also show that the temperature is controlled very well during the simulation process. 
Several reports on the toxicity of nanomaterials are available that summarize current knowledge that we have and highlight areas that raise concerns. ${ }^{[27-29]}$ However, most of the strategies suggested in these reports are based on inherently experimental approaches. It is widely accepted that the experimental results may be very sensitive to a wide range of physical properties of the nanoparticles such as the size, the shape, the surface structure and the chemical composition. They are also affected largely by the environment- the solvent, the solute ions, the surfactants, and the temperature and so on. Some of the factors may be out of experimental control. Moreover, these dependencies are intrinsically linked, and one has to take this into account before making any reliable predictions about the potential risks of having these materials in a biological system.

Considering that there are certain limitations using solely the experimental approaches in the study of cytotoxicity of nanomaterials. MD or DFT simulations certainly have advantage in controlling each of these critical parameters independently in order to identify underlying mechanisms responsible for each of the factors involved in the process. In our study, through the simulation of the interaction of AuNPs or AgNPs with a membrane, we can clearly evaluate to what degree the AuNPs or AgNPs affect the function of the cell membrane. We found that both AuNPs and AgNPs have a strong interaction with the hydrophilic groups. As a result, the hydrophilic headgroup sticks to the surface of the nanoparticles and the membrane is largely deformed by this interaction. As one can imagine this will inevitably lead to the mis-function of these biomolecules, it 
is known that it is critical for the lipid molecules to maintain sufficient flexibility in order to retain their normal function of the membrane.

The biological membrane is the collage of protein and other molecules embedded in the fluid matrix of the lipid bilayer. The flexibility of lipid molecules allows the globular protein to rotate in the fluid matrix and to bring useful nutrient substances from outside to inside the cell to retain a normal cell metabolism. Any factor that makes a change in this flexibility can lead to the occurrence of toxicity for the membrane. Based on these arguments, we can say that AuNPs and AgNPs can have pronounced toxicity in disturbing the normal functionality of the cell membrane.

Earlier experimental reports indicate that cellular uptake and cytotoxicity of the AuNPs are in fact two linked factors which are jointly controlled by the absorption ability of the AuNPs. Our simulation results give further information on how these factors are related to each other. The electronic structure of Gold atom is $[\mathrm{Xe}] 4 \mathrm{f}^{14} 5 \mathrm{~d}^{10} 6 \mathrm{~s}^{1}$ and it has an unpaired $6 \mathrm{~s}$ electron. These types of elements possess strong coordination ability with organic molecules. Through coordination interaction, they usually lead to relatively strong chemisorption. In phospholipid compounds, the amine, phosphine or carboxyl chemical functional groups chemically interact with metallic gold atoms. Furthermore, nanoparticles have a very large specific surface ratio and tend to have a strong adsorption capacity. These factors make the hydrophilic part in the phospholipid molecules adsorbed firmly to the surface of AuNPs. Since adsorption occurs locally, all the phospholipid molecules which lie within the interaction range will be firmly attached to the surface of 
nanoparticles. The other neighboring phospholipid molecules will probably be less connected with this hump. Therefore, the phospholipid layer interacting with AuNPs can result in avulsion of the splenic substance.

As we can see, the gold nanoparticles can cause cell membrane tearing and change movement speed of phospholipid molecular layer in cell membranes; it will affect the normal biological membrane function. It remains a challenge how to measure this effect. We now look into using the Root Mean Square Deviation (RMSD) value to measure the toxicity of nanoparticles based on the above analysis. These interacting phospholipid molecules are expected to have different velocities (or flexibility) as compared with the non-interacting ones.

Statistically speaking, RMSD is equivalent to the standard deviation, which reflects the deviation degree from the mean for a set of data, and is most commonly used to measure the change in atomic positions in simulation studies. In this work, we have carried out the RMSD analysis of the position of the lipid bilayer. The RMSD value reflects the deviation degree of the molecule from its average position over a period of time, that is, the special range of motion of the molecule. A larger RMSD value indicates that the spatial extent of the movement of the molecule is larger, thereby a larger flexibility in the molecule. RMSD is calculated as follows:

$$
\operatorname{RMSD}_{\alpha}\left(t_{j}\right)=\sqrt{\frac{\sum_{\alpha=1}^{N_{\alpha}}\left(\vec{r}_{\alpha}\left(t_{j}\right)-<\vec{r}_{\alpha}>\right)^{2}}{N_{\alpha}}}
$$


where $N_{\alpha}$ is the number of atoms in a molecule involved in the comparison, $\vec{r}_{\alpha}\left(t_{j}\right)$ is the position of the $\alpha$ atom in the molecule at time $t_{j},\left\langle\vec{r}_{\alpha}\right\rangle$ is the average position of the $\alpha$ atom in the entire period and it is defined as:

$$
<\vec{r}_{\alpha}>=\frac{1}{N_{t}} \sum_{j=1}^{N_{t}} \vec{r}_{\alpha}\left(t_{j}\right)
$$

As discussed previously, the velocity of lipid molecules in a membrane is very important for the function of the membrane. How could one quantify this velocity for each lipid molecule then? According to the definition of RMSD, it is apparent that the RMSD value actually is correlated with the physical quantity of speed indicating the rate of change in position. Therefore, in the following we will use the RMSD results to discuss the cytotoxicity of nanoparticles. All the computed RMSD values for all lipid molecules $(8 * 8)$ are listed in Tables 7.3 and 7.4, for AuNPs and AgNPs, respectively. In order to compare with the non-interacting side, the RMSD values for the bottom monolayer of the lipid are also plotted together with the interacting top monolayer in Figures 7.9 and 7.10, for AuNPs and AgNPs, respectively. 
Table 7.3: Computed RMSD values for each DMPC molecule in the top monolayer (only this layer is considered to interact with AuNPs).

\begin{tabular}{|c|c|c|c|}
\hline $\begin{array}{l}\text { No. of } \\
\text { molecule }\end{array}$ & $\begin{array}{c}\mathrm{X} \\
\text { coordinate of } \\
\mathrm{N} \text { atom }\end{array}$ & $\begin{array}{l}\text { Y coordinate } \\
\text { of } \mathrm{N} \text { atom }\end{array}$ & RMSD $(\AA)$ \\
\hline 1 & -16.813 & 16.338 & 4.2951 \\
\hline 2 & -25.630 & -26.379 & 3.2313 \\
\hline 3 & 16.063 & -11.324 & 4. 1461 \\
\hline 4 & -19.033 & 26.558 & 3.3610 \\
\hline 5 & -16.567 & -30.495 & 3.6232 \\
\hline 6 & -7.671 & -0.832 & 4. 3104 \\
\hline 7 & 2.907 & -20.462 & 3.9504 \\
\hline 8 & -13.861 & -20.781 & 3.6310 \\
\hline 9 & 23.317 & 9.573 & 4. 9991 \\
\hline 10 & 7. 202 & -11.173 & 3.9466 \\
\hline 11 & -17.105 & -11.524 & 3.6959 \\
\hline 12 & -16.968 & 4. 133 & 3.8732 \\
\hline 13 & 21.192 & 23.188 & 3.9008 \\
\hline 14 & 20.700 & 2.966 & 4.5661 \\
\hline 15 & -6.912 & -12.730 & 3.7039 \\
\hline 16 & -7.044 & 19.612 & 3.6524 \\
\hline 17 & 10.343 & 1.559 & 3.7162 \\
\hline 18 & 17.713 & -21.985 & 3.6557 \\
\hline 19 & 21.905 & -19.935 & 3.2741 \\
\hline 20 & 0.566 & 26.454 & 3.9742 \\
\hline 21 & -20.310 & 22.560 & 3.9088 \\
\hline 22 & -14.071 & 8.813 & 4.3448 \\
\hline 23 & -29.943 & -3.021 & 3.6582 \\
\hline
\end{tabular}




\begin{tabular}{|c|c|c|c|}
\hline 24 & -23.393 & -3.409 & 3.4811 \\
\hline 25 & 19.572 & 16.547 & 4. 0449 \\
\hline 26 & 5.326 & 9.405 & 3.1644 \\
\hline 27 & -13.558 & -7.091 & 3.1467 \\
\hline 28 & 11.969 & 16.639 & 4.2795 \\
\hline 29 & -29.611 & -16.642 & 3.7659 \\
\hline 30 & 30.345 & 2.372 & 3.9135 \\
\hline 31 & 9.578 & -19.718 & 4. 3054 \\
\hline 32 & -16.387 & 29.990 & 4. 0755 \\
\hline 33 & 25.229 & -26.292 & 3.1644 \\
\hline 34 & 5.634 & 19.933 & 4.2201 \\
\hline 35 & 17.068 & -5.365 & 3.2062 \\
\hline 36 & -25.720 & 14.962 & 5.0403 \\
\hline 37 & 25.370 & 24.302 & 4. 1340 \\
\hline 38 & -30.901 & 23.830 & 5.0971 \\
\hline 39 & 15.649 & 9.152 & 3.7056 \\
\hline 40 & -3.307 & -27.133 & 3.8990 \\
\hline 41 & 5.700 & -3.560 & 2.4637 \\
\hline 42 & 20.159 & -3.122 & 6.5519 \\
\hline 43 & 28.248 & 14.407 & 3.2136 \\
\hline 44 & -0.827 & 11.135 & 3.7531 \\
\hline 45 & 32.177 & 30.326 & 4.1281 \\
\hline 46 & -5.925 & -27.417 & 4.2070 \\
\hline 47 & 8.043 & -27.470 & 4.0883 \\
\hline 48 & 15.485 & -29.465 & 3.9034 \\
\hline 49 & -4.091 & 5.437 & 4.3749 \\
\hline 50 & 1.725 & -12.742 & 4. 0710 \\
\hline 51 & -1.158 & -4.419 & 4.8789 \\
\hline
\end{tabular}




\begin{tabular}{|c|c|c|c|}
\hline 52 & -26.949 & 1.134 & 2.7634 \\
\hline 53 & -4.142 & -20.641 & 4.7187 \\
\hline 54 & 2.414 & 5.013 & 3. 9519 \\
\hline 55 & 22.915 & 31.710 & 4.1105 \\
\hline 56 & -13.093 & 22.368 & 5.0831 \\
\hline 57 & -21.092 & -18.273 & 3.7847 \\
\hline 58 & 29.246 & -11.325 & 3.6463 \\
\hline 59 & 10. 055 & 24.872 & 4.4514 \\
\hline 60 & -29.297 & -12.653 & 3.5570 \\
\hline 61 & -22.681 & 9.063 & 4.0421 \\
\hline 62 & -29.512 & 9.298 & 3.8057 \\
\hline 63 & -5.034 & 27.235 & 4.4051 \\
\hline 64 & -12.240 & 14.251 & 4.4411 \\
\hline 65 & -3.779 & 7. 365 & -4.0128 \\
\hline 66 & 2.506 & 9.492 & -3.2397 \\
\hline 67 & -24.307 & -24.757 & -3.6179 \\
\hline 68 & -17.953 & 1.255 & -4.0871 \\
\hline 69 & 24.202 & -22.336 & -3.3337 \\
\hline 70 & 31.401 & 27.359 & -3.4572 \\
\hline 71 & -17.791 & 19.333 & -3.1679 \\
\hline 72 & -10.734 & 29.463 & -3.7645 \\
\hline 73 & -26.055 & -0.854 & -3.5470 \\
\hline 74 & 21.761 & -11.048 & -3.8982 \\
\hline 75 & -16.262 & -10.107 & -3.9648 \\
\hline 76 & 2.734 & -7.009 & -3.7006 \\
\hline 77 & -25.192 & -10.353 & -3.6065 \\
\hline 78 & 32.105 & 1.750 & -3.2678 \\
\hline 79 & -3.159 & 23.297 & -5.1906 \\
\hline
\end{tabular}




\begin{tabular}{|c|c|c|c|}
\hline 80 & -4.535 & -3.229 & -4.6360 \\
\hline 81 & 13. 772 & 30.608 & -3.1595 \\
\hline 82 & 7.675 & 23.576 & -3.2206 \\
\hline 83 & -24.336 & 19.926 & -3.5694 \\
\hline 84 & 18.543 & 15.662 & -4.8659 \\
\hline 85 & 16.187 & -22.810 & -3.0367 \\
\hline 86 & 0.071 & -13.938 & -3.3362 \\
\hline 87 & -14.920 & -29.837 & -3.0069 \\
\hline 88 & 10.094 & -5.083 & -3.5511 \\
\hline 89 & 2.669 & 19.653 & -3.7643 \\
\hline 90 & 17.088 & -16.683 & -4.6027 \\
\hline 91 & 11.208 & -11.098 & -3.4632 \\
\hline 92 & 31.396 & 9.003 & -3.7925 \\
\hline 93 & -13.326 & -19.965 & -3.3876 \\
\hline 94 & -18.762 & 10.302 & -3.8768 \\
\hline 95 & -10.490 & -11.056 & -3.4093 \\
\hline 96 & 16.567 & 1.781 & -3.0594 \\
\hline 97 & -23.294 & 4.505 & -3.4346 \\
\hline 98 & 9.786 & 13.002 & -4.4030 \\
\hline 99 & -2.080 & 16.609 & -3.9241 \\
\hline 100 & -1.475 & -32.140 & -3.8523 \\
\hline 101 & -8.008 & -26.735 & 3.4700 \\
\hline 102 & 28.167 & -24.992 & -3.3894 \\
\hline 103 & -21.181 & -17.784 & -3.6930 \\
\hline 104 & 1.094 & -24.363 & -3.7268 \\
\hline 105 & 30.268 & 18.509 & -3.7606 \\
\hline 106 & -18.740 & 27.366 & -3.8678 \\
\hline 107 & -24.029 & 15.318 & -3.2429 \\
\hline
\end{tabular}




\begin{tabular}{|c|c|c|c|}
\hline 108 & -11.291 & 12.982 & -3.2517 \\
\hline 109 & 10. 941 & 7.578 & -3.0747 \\
\hline 110 & 26.713 & -8.534 & -4.3257 \\
\hline 111 & 21.368 & 27.423 & -3.8929 \\
\hline 112 & 2.336 & 1.601 & -4.2736 \\
\hline 113 & -5.019 & -9.785 & -3.9242 \\
\hline 114 & -10.495 & -2.244 & -3.0736 \\
\hline 115 & -24.276 & 32.642 & -3.5813 \\
\hline 116 & -12.525 & 19.751 & -3.8781 \\
\hline 117 & -9.191 & 4. 400 & -3.5093 \\
\hline 118 & 8.332 & -28.516 & -3.3791 \\
\hline 119 & -4.787 & -17.178 & -3.4974 \\
\hline 120 & 14.623 & -8.714 & -3.4627 \\
\hline 121 & 8.449 & -19.161 & -4.1167 \\
\hline 122 & 24. 043 & -1.945 & -3.0771 \\
\hline 123 & 6.036 & 27.056 & -4.0758 \\
\hline 124 & 25.966 & 5.793 & -2.9996 \\
\hline 125 & 14.696 & 10.187 & -3.3459 \\
\hline 126 & 24.360 & 13.314 & -3.8626 \\
\hline 127 & 30.595 & -19.840 & -3.8484 \\
\hline 128 & 22.478 & -30.626 & -4.0212 \\
\hline
\end{tabular}


Table 7.4: Computed RMSD values for each DMPC molecule in the top monolayer (only this layer is considered to interact with AgNPs).

\begin{tabular}{|c|c|c|c|}
\hline $\begin{array}{r}\text { No. of } \\
\text { molecule }\end{array}$ & $\begin{array}{l}\mathrm{X} \text { coordinate of } \\
\mathrm{N} \text { atom }\end{array}$ & $\begin{array}{l}\mathrm{Y} \text { coordinate } \\
\text { of } \mathrm{N} \text { atom }\end{array}$ & $\operatorname{RMSD}(\AA)$ \\
\hline 1 & -16.813 & 16.338 & 3.1819 \\
\hline 2 & -25.630 & -26.379 & 3.6814 \\
\hline 3 & 16.063 & -11.324 & 3.7606 \\
\hline 4 & -19.033 & 26.558 & 3.7978 \\
\hline 5 & -16.567 & -30.495 & 4.0126 \\
\hline 6 & -7.671 & -0.832 & 4.3998 \\
\hline 7 & 2.907 & -20.462 & 3.2376 \\
\hline 8 & -13.861 & -20.781 & 3.5472 \\
\hline 9 & 23.317 & 9.573 & 3.2995 \\
\hline 10 & 7.202 & -11.173 & 3.1755 \\
\hline 11 & -17.105 & -11.524 & 3.7124 \\
\hline 12 & -16.968 & 4.133 & 3.5657 \\
\hline 13 & 21.192 & 23.188 & 3.8560 \\
\hline 14 & 20.700 & 2.966 & 4.0099 \\
\hline
\end{tabular}




\begin{tabular}{|c|c|c|c|}
\hline 15 & -6.912 & -12.730 & 5.5326 \\
\hline 16 & -7.044 & 19.612 & 3.4712 \\
\hline 17 & 10.343 & 1.559 & 3.2789 \\
\hline 18 & 17.713 & -21.985 & 3.2496 \\
\hline 19 & 21.905 & -19.935 & 4.4799 \\
\hline 20 & 0.566 & 26.454 & 4.3663 \\
\hline 21 & -20.310 & 22.560 & 3.1328 \\
\hline 22 & -14.071 & 8.813 & 3.5889 \\
\hline 23 & -29.943 & -3.021 & 3.2258 \\
\hline 24 & -23.393 & -3.409 & 3.6057 \\
\hline 25 & 19.572 & 16.547 & 3.1996 \\
\hline 26 & 5.326 & 9.405 & 3.9738 \\
\hline 27 & -13.558 & -7.091 & 3.6296 \\
\hline 28 & 11.969 & 16.639 & 4.5259 \\
\hline 29 & -29.611 & -16.642 & 3.7271 \\
\hline 30 & 30.345 & 2.372 & 3.6654 \\
\hline 31 & 9.578 & -19.718 & 3.7069 \\
\hline
\end{tabular}




\begin{tabular}{|c|c|c|c|}
\hline 32 & -16.387 & 29.990 & 3.0469 \\
\hline 33 & 25.229 & -26.292 & 3.2099 \\
\hline 34 & 5.634 & 19.933 & 4.2425 \\
\hline 35 & 17.068 & -5.365 & 3.8114 \\
\hline 36 & -25.720 & 14.962 & 3.9820 \\
\hline 37 & 25.370 & 24.302 & 3.2895 \\
\hline 38 & -30.901 & 23.830 & 3.1823 \\
\hline 39 & 15.649 & 9.152 & 3.9367 \\
\hline 40 & -3.307 & -27.133 & 4.3138 \\
\hline 41 & 5.700 & -3.560 & 3.7814 \\
\hline 42 & 20.159 & -3.122 & 3.7422 \\
\hline 43 & 28.248 & 14.407 & 3.6192 \\
\hline 44 & -0.827 & 11.135 & 3.2858 \\
\hline 45 & 32.177 & 30.326 & 3.4011 \\
\hline 46 & -5.925 & -27.417 & 4.4553 \\
\hline 47 & 8.043 & -27.470 & 3.6861 \\
\hline 48 & 15.485 & -29.465 & 4.9452 \\
\hline
\end{tabular}




\begin{tabular}{|c|c|c|c|}
\hline 49 & -4.091 & 5.437 & 3.7232 \\
\hline 50 & 1.725 & -12.742 & 4.0360 \\
\hline 51 & -1.158 & -4.419 & 3.7256 \\
\hline 52 & -26.949 & 1.134 & 3.2382 \\
\hline 53 & -4.142 & -20.641 & 3.2382 \\
\hline 54 & 2.414 & 5.013 & 2.9179 \\
\hline 55 & 22.915 & 31.710 & 3.9964 \\
\hline 56 & -13.093 & 22.368 & 2.9965 \\
\hline 57 & -21.092 & -18.273 & 3.6594 \\
\hline 58 & 29.246 & -11.325 & 2.9791 \\
\hline 59 & 10.055 & 24.872 & 4.1276 \\
\hline 60 & -29.297 & -12.653 & 3.6913 \\
\hline 61 & -22.681 & 9.063 & 3.6913 \\
\hline 62 & -29.512 & 9.298 & 4.4244 \\
\hline 63 & -5.034 & 27.235 & 4.7395 \\
\hline 64 & -12.240 & 14.251 & 3.8395 \\
\hline
\end{tabular}




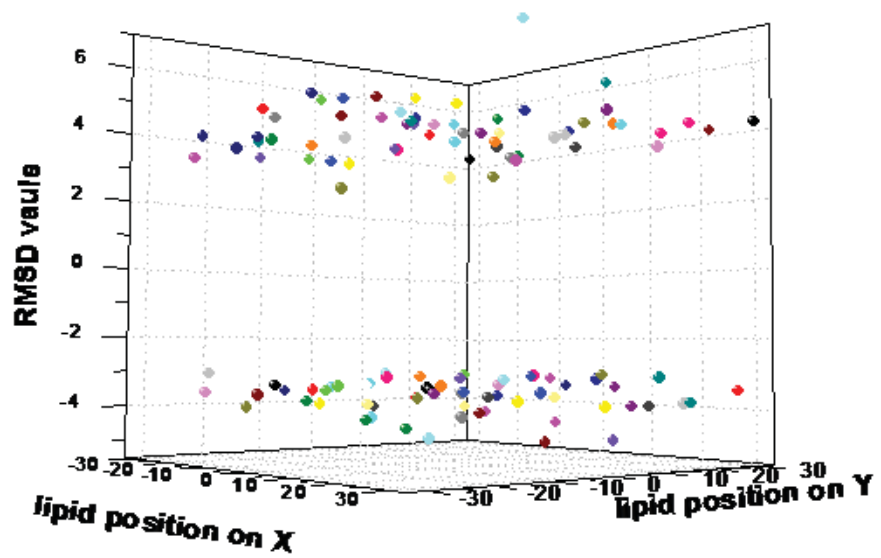

Figure 7.9: A plot of the RMSD values of the DMPC molecules in the membrane interacting with AuNPs.

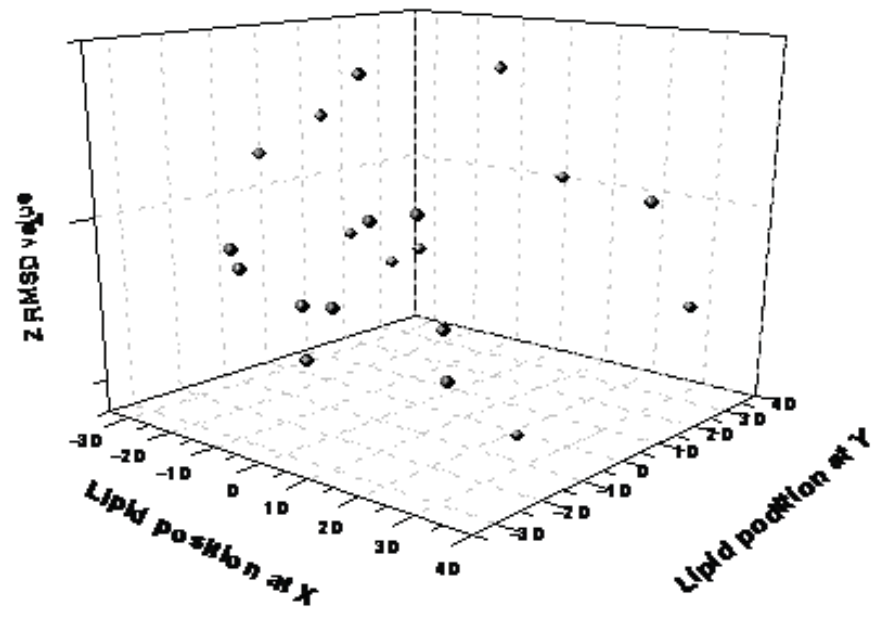

Figure 7.10: A plot of the RMSD values of the DMPC molecules in the membrane interacting with AgNPs. 
Figures 7.10 and 7.11 suggest that the RMSD values are larger for the top lipid layer interacting with AuNPs or AgNPs than for the lower layer on the other side of the lipid. Considering that the larger the RMSD numerical difference is made by the NPs, the more toxicity effects are likely to occur for nanoparticles interacting with cell membranes, we find that gold nanoparticles can cause a relatively strong toxicity to the cell membrane considered.

\subsection{Summary}

We have studied the interactions of AuNPs and AgNPs with model lipid membranes using MD simulations. For the first time, a new force field has been developed to describe the interaction between metal NPs and cell membranes which is primarily governed by the coordination chemistry. The results show that AuNPs rapidly penetrate into the headgroup region of the lipid, then adheres to the hydrophilic part of the phospholipid bilayer owing to the strong interaction between AuNPs/AgNPs and the active functional groups of the lipid bilayer, including amine, phosphine and carboxyl chemical groups. The permeation of AuNPs/AgNPs into the lipid membrane and its concomitant membrane disruption discovered in the simulation indicate that AuNPs have cytotoxicity. It is verified by the observation of the bypass of the endocytosis pathway upon their internalization into cells in experiments. It is also found that the phospholipid layer can result in avulsion of the splenic substance under the interaction with AuNPs/AgNPs. 
The RMSD analysis is proved to be a useful tool in quantifying the disturbance of the cell membrane by the intrusion of AuNPs/AgNPs. Our computed RMSD values suggest that both AuNPs and AgNPs have relatively large impact on the phospholipid molecular layer with the AgNPs being slightly weaker. As a measure of the toxicity, this result shows that the gold and silver nanoparticles may have a strong toxicity to the cell membrane. These results provide critical information for the safely use of AuNPs and AgNPs to achieve designated goals in their delivery, diagnostic, and therapeutic applications. 


\section{References}

[1] Xia, Younan, et al. "One - dimensional nanostructures: synthesis, characterization, and applications." Advanced materials 15.5 (2003) 353-389.

[2] $\mathrm{Xu}$, Terry T., et al. "Bone-shaped nano $\{\mathrm{Xu}, 2003 \# 2590\}$ materials for nanocomposite applications." Nano Letters 3.8 (2003) 1135-1139.

[3] Pal, Sukdeb, Yu Kyung Tak, and Joon Myong Song. "Does the antibacterial activity of silver nanoparticles depend on the shape of the nanoparticle? A study of the gram-negative bacterium Escherichia coli." Applied and environmental microbiology 73.6 (2007) 1712-1720.

[4] Chen, Jiong, et al. "Effect of silver nanoparticle dressing on second degree burn wound." Zhonghua wai ke za zhi [Chinese journal of surgery] 44.1 2006, 50-53.

[5] You, Chuangang, et al. "The progress of silver nanoparticles in the antibacterial mechanism, clinical application and cytotoxicity." Molecular biology reports 39.9 (2012) 9193-9201.

[6] Kim, Yong Soon, et al. "Twenty-eight-day oral toxicity, genotoxicity, and genderrelated tissue distribution of silver nanoparticles in Sprague-Dawley rats." Inhalation toxicology, 20.6 (2008) 575-583. 
[7] Ghosh, Partha, et al. "Gold nanoparticles in delivery applications." Advanced drug delivery reviews 60.11 (2008) 1307-1315.

[8] Cheng, Yu, et al. "Highly efficient drug delivery with gold nanoparticle vectors for in vivo photodynamic therapy of cancer." Journal of the American Chemical Society 130.32 (2008) 10643-10647.

[9] Sandhu, Kulmeet K., et al. "Gold nanoparticle-mediated transfection of mammalian cells." Bioconjugate chemistry 13.1 (2002) 3-6.

[10] Huang, Xiaohua, et al. "Cancer cells assemble and align gold nanorods conjugated to antibodies to produce highly enhanced, sharp, and polarized surface Raman spectra: a potential cancer diagnostic marker." Nano letters 7.6 (2007) 1591-1597.

[11] Wang, Zhenxin, et al. "Microarray-based detection of protein binding and functionality by gold nanoparticle probes." Analytical chemistry 77.17 (2005) 5770-5774.

[12] Cho, E. C.; Xie, J.; Wurm, P. A.; Xia, Y. "Understanding the Role of Surface Charges in Cellular Adsorption versus Internalization by Selectively Removing Gold Nanoparticles on the Cell Surface with a I2/KI Etchant". Nano Lett. 9, (2009) 1080-1084.

[13] Goodman, C. M.; McCusker, C. D.; Yilmaz, T.; Rotello, V. M. "Toxicity of Gold Nanoparticles Functionalzied with Cationic and Anionic Side Chians". Bioconjugate Chem. 15, (2004) 897-900. 
[14] Chithrani, B. D.; Ghazani, A. A.; Chan, W. C. "Determining the Size and Shape Dependence of Gold Nanoparticle Uptake into Mam- malian Cells”. Nano Lett. 6, (2006) $662-668$.

[15] Lewinski, N.; Colvin, V.; Drezek, R. "Cytotoxicity of Nanoparticles”. Small, 4, (2008) 26-49.

[16] Leroueil, Pascale R., et al. "Wide varieties of cationic nanoparticles induce defects in supported lipid bilayers." Nano letters 8.2 (2008) 420-424.

[17] Lee, Kerry J., et al. "In vivo imaging of transport and biocompatibility of single silver nanoparticles in early development of zebrafish embryos." ACS nano 1.2 (2007) $133-143$.

[18] Leroueil, P. R.; Berry, S. A.; Duthie, K.; Han, G.; Rotello, V. M.; McNerny, D. Q.; Baker, J. R.; Orr, B. G.; Holl, M. M. "Wide Varieties of Cationic Nanoparticles Induce Defects in Supported Lipid Bilayers". Nano Lett. 8, (2008) 420-424.

[19] Wong-Ekkabut, Jirasak, et al. "Computer simulation study of fullerene translocation through lipid membranes." Nature nanotechnology 3.6 (2008) 363-368.

[20] Bedrov, Dmitry, et al. "Passive transport of C60 fullerenes through a lipid membrane: a molecular dynamics simulation study." The Journal of Physical Chemistry B 112.7 (2008) 2078-2084. 
[21] Li, Liwei, et al. "A molecular dynamics simulation study of C60 fullerenes inside a dimyristoylphosphatidylcholine lipid bilayer." The Journal of Physical Chemistry B 111.16 (2007) 4067-4072.

[22] Wallace, E. Jayne, and Mark SP Sansom. "Carbon nanotube self-assembly with lipids and detergent: a molecular dynamics study." Nanotechnology 20.4 (2009) 045101.

[23] Hong, Seungpyo, et al. "Interaction of poly (amidoamine) dendrimers with supported lipid bilayers and cells: hole formation and the relation to transport."Bioconjugate chemistry 15.4 (2004) 774-782.

[24] Lin, Jiaqi, et al. "Penetration of lipid membranes by gold nanoparticles: insights into cellular uptake, cytotoxicity, and their relationship." ACS nano 4.9 (2010) 5421-5429.

[25] Lin, Jia-Qi, et al. "A simulation study on nanoscale holes generated by gold nanoparticles on negative lipid bilayers." Langmuir 27.13 (2011) 8323-8332.

[26] Authors: M. Bhandarkar, A. Bhatele, E. Bohm, R. Brunner, F. Buelens, C. Chipot, A. Dalke, S. Dixit, G. Fiorin, P. Freddolino, P. Grayson, J. Gullingsrud, A. Gursoy, D. Hardy, C. Harrison, J. H'enin, W. Humphrey, D. Hurwitz, N. Krawetz, S. Kumar, D. Kunzman, J. Lai, C. Lee, R. McGreevy, C. Mei, M. Nelson, J. Phillips, O. Sarood, A. Shinozaki, D. Tanner, D. Wells, G. Zheng, F. Zhu Theoretical Biophysics Group, Beckman Institute, University of Illinois. 
[27] Barnard, Amanda S. "How can ab initio simulations address risks in nanotech?." Nature nanotechnology 4.6 (2009) 332-335.

[28] Maynard, Andrew D., David B. Warheit, and Martin A. Philbert. "The new toxicology of sophisticated materials: nanotoxicology and beyond." Toxicological Sciences 120.suppl 1 (2011) S109-S129.

[29] Barnard, Amanda S. "Computational strategies for predicting the potential risks associated with nanotechnology." Nanoscale 1.1, (2009) 89-95. 


\section{Chapter 8}

\section{Summary and Future Work}

The great advances in nanotechnology and the vast use of nanomaterials have brought both exciting applications and serious potential health and environmental concerns. The active nanoparticles are within the same size range of biological molecules. The interaction between these two - inorganic and organic materials becomes a topic of dramatic interest. In the past several years, I have tried to bring physics, nanotechnology and biology together. In the following (Figure 8.1: boxes with normal fonts), I have summarized the work I have attempted in this field. It is a very interesting, important and challenging field, to my opinion. Besides the work I have done, there is actually a lot more work that needs to be done (Figure 8.1: boxes with bold fonts). 


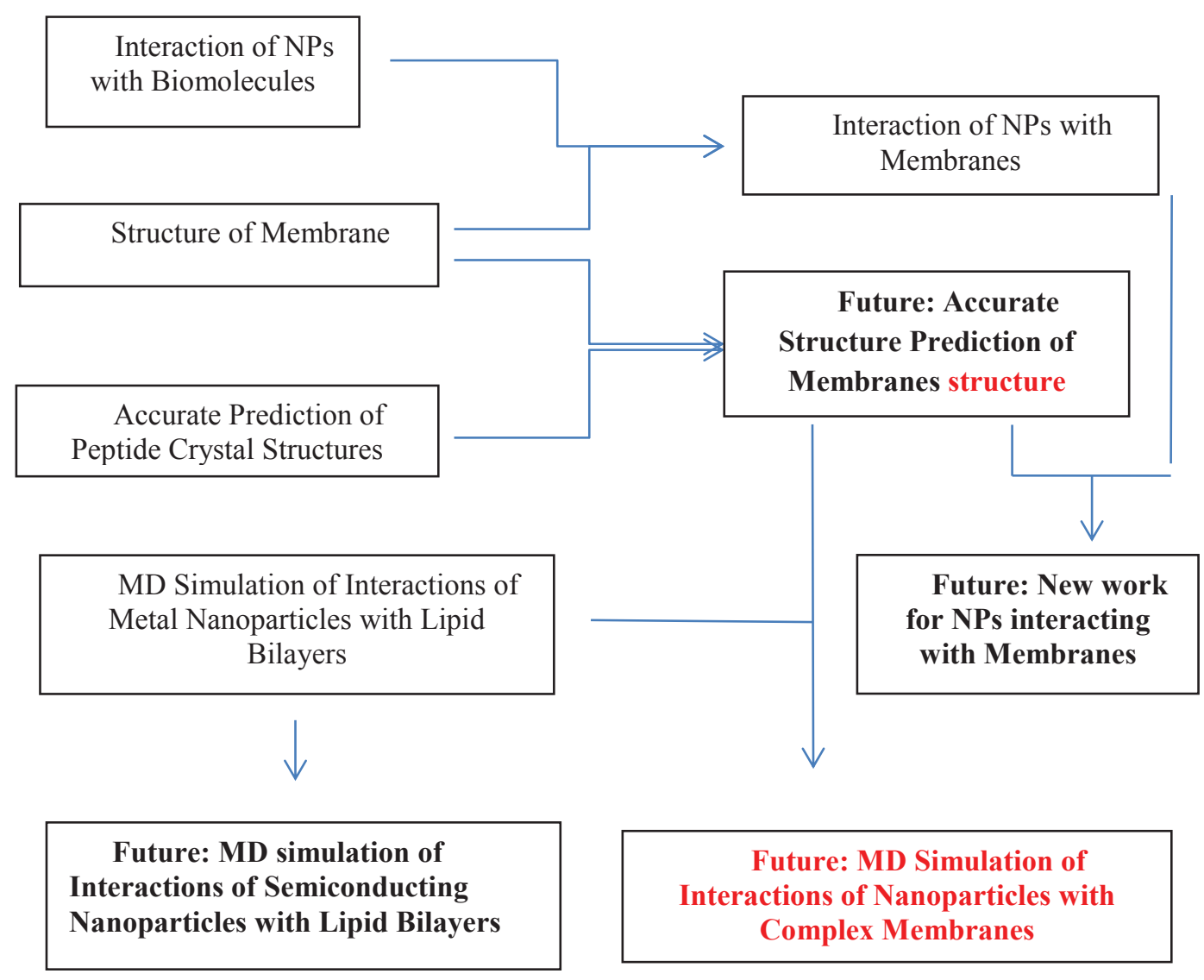

Figure 8.1: Summary of completed work and future directions.

The Quantum mechanical approach provides high accuracy in describing the interaction between atoms, charge transfers and natures of different bonding, but it comes with a high computational cost. The current state of the art is that they can only be applied to several hundred of atoms. The classical molecular dynamic approach is able to tackle millions of atoms, but with less atomic level details and the reliability is highly subject to the employed force fields describing the atomistic interactions. The system that we are mostly interested in is small enough which demands an accurate account of the 
charge distribution and the bonding at the atomic level, but too big to be treated completely using quantum mechanical approach. A combination of the density functional theory and the molecular dynamic force field is used to get a more accurate description of individual systems as well as to investigate the nano-bio interactions. It is a great attempt to conduct a multi-scale modeling. Significant efforts have been taken to develop proper simulation models. The limitation in the modeling and simulation and proposed future directions are discussed in the following.

As we can see from the computational simulation results in Chapter 6, the accurate three-dimensional structure of a peptide crystal can be obtained from a full firstprinciples approach by knowledge of the chemical and bonding symmetry consideration in a very systematic way. Future work can be done to apply this method to other peptide crystals to further validate the method and establish its generality.

The lipid structure (Chapter 5) can be improved by including the van der Waals interactions, which was lacking in the software we used at the moment of our simulation. Our later study of peptide crystals, together with other studies have shown that van der Waals forces are very important for accurately predicting the crystal structure of organic or biological molecules, owing to the significant intermolecular interactions. Another challenge comes from the simulation of lipid structure at experimentally-comparable temperature, which is usually around room temperature. We know for inorganic materials the thermal expansion coefficient is usually very low, this is not the case for the biological system. Ab initio molecular dynamics may help in this case. The so-obtained 
new structure may then be used to study its interaction with the nanoparticles using either first principles or molecular dynamics method.

For the MD simulation of metal nanoparticles interacting with membranes (Chapter 8), we have taken several simplifications in our model. First, the metal nanoparticle is simulated by a $1-\mathrm{nm}$ cluster. The study can be extended to different sized particles to see the size effect. Secondly, the membrane is modeled purely by a phospholipid bilayer. We know proteins exist on the cell membranes and are also very important in the functioning of membranes. How the NPs interacting with a protein or a protein in a membrane would be a very interesting aspect to look at. Therefore, a lot more complexity can be added to the model of the cell membrane. Last, but not the least, we know the interaction is system-dependent (Chapters 3, 4 and 8). It would be greatly beneficial to expand the current methodology to studies of other metallic and semiconducting nanomaterials, which are also of practical interest.

\section{Yes, interesting scientific research has not ended, but rather just began.}




\section{The List of the Selected Publications}

1. C. Liu, H. He, R. Pandey, S. Hussain, and S. P. Karna, "Interaction of Metallic Nanoparticles with Biological Active Molecule Dopamine", Journal of Physical Chemistry B, 2008, 112 (47), 15256-15259.

2. Prashant K. Sharma, Ranu K. Dutta, Chun Hui Liu, Ravindra Pandey, Avinash C. Pandey, "Surfactant mediated optical properties of cytosine capped CdSe quantum dots” , Materials Letters, 2010, 64(10), 1183 - 1186.

3. Chunhui Liu, Haiying He, Ravindra Pandey, and Shashi P. Karna, "From Molecules to Crystals- First Principles Prediction of Peptide Crystal Structures", (in preparation).

4. Chunhui Liu, Haiying He, Ravindra Pandey, and Shashi P. Karna, “A simulation study on gold nanoparticles interaction with lipid bilayers", (in preparation). 